\section{VIBRATION STUDY OF THE APS STORAGE RING 0.8 METER QUADRUPOLE/GIRDER ASSEMBLY}

ANL/APS/IN/VIB--91/2

DE92 012172

by

\section{J. A. Jendrzejczyk, M. W. Wambsganss, and R. K. Smith Materials and Components Technology Division}

Argonne National Laboratory

\title{
BACKGROUND
}

A vibration study of the APS magnet support assembly was reported by Wambsganss et al. [1]. The storage ring magnets were simulated by concrete shielding blocks and test and analytical results were obtained relating to vibration of the APS storage ring section 3 girder. Subsequently, an additional vibration study of the storage ring vacuum chamber/section 3 girder was completed and reported [2]. In the latter study, the vacuum chamber was mounted to the section 3 girder with prototypic supports, and vibration measurements were made at the beam position monitor (BPI) locations. Due to the required test configuration, the magnet weights could not be easily simulated. The girder assembly was not anchored to the floor due to the small mass ratio of the vacuum chamber to the girder, but the turnbuckles were tight to remove clearance from the jackscrew assembly and also to provide some load to the girder.

As discussed in [1], the quadrupole magnets have very stringent vibration level criteria that must be satisfied if successful operation of the facility is to be achieved. If a vibration mode of a quadrupole magnet coincides with a vibration mode of the girder, a large transfer function, with the possibility of large displacements at the magnet, can occur. Depending on frequency and amplitude, the feedback control system and steering magnets may not be able to compensate. In studying the coupled modes of the magnet/girder assembly, it is necessary to duplicate, as closely as practical, the actual girder loading and floor anchoring to insure an accurate system frequency response.

\section{OBJECTIVES}

The overall objective of this study is of obtain insights into the dynamic coupled behavior of the quadrupole magnet and the girder assembly, and an assessment of the potential for unacceptable vibration levels which would rocuire redesign of the quadrupole and/or girder mounting system(s).

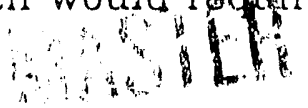


Specific objectives irclude determination of vibrational characteristics (natural frequencies, damping, mode shapes, and transfer functions) of the coupled magnet/girder system, measurement of response amplitudes to forced excitation and ambient floor motion, and calculation of magnification factors associated with the observed coupled vibration modes.

\section{TECHNICAL APPROACH}

A prototypic $0.8 \mathrm{~m}$ quadrupole magnet with prototypic jackscrew supports is mounted on the section 3 girder. Since we are measuring the coupled vibration frequencies associated with the magnet and girder systems, it is desirable to have the prototypic system model simulate the actual one as close as possible. Consequently, concrete shielding blocks, similar to those used in the study of Ref. 1 , are used to simulate the weight of the other magnets and components. The blocks are aligned in a horizontal orientation to within 0.4 in of the actual magnet position. However, due to the dimensions of the blocks, the center of mass is lower. Since elevating the blocks on stand-offs has been shown to result in extraneous frequencies that are not prototypic [1], the blocks were set directly on the girder. The jackscrews were set to provide a girder elevation of 34 inches, and were bolted to the floor. For the Phase I tests (to be defined below), the turnbuckles between the girder and jackscrew positioning frame were tighi.

The actual testing was completed in two phases. In the Phase I tests (Tests 1-7), the 0.8 meter quadrupole was mounted to the girder with a prototypic mount and excitation was primarily by an electromagnetic exciter or the result of ambient floor motion, with the exception of Test 7 , which was impulse excited. In the Phase II tests (Tests 8-15) the excitation was primarily by impulse with only a few tests with ambient floor excitrtion.

Magnet resonant frequencies were determined by placing the exciter on the top of the magnet and exciting the magnet with a random force excitation (0$150 \mathrm{~Hz}$ ) in all three axes. The random force was then applied to the girder to determine transfer functinns between it and the magnet. Force impulse excitation was used to masure system damping, while sine excitation was used in an attempt to identify the vibration mode shapes of the girder/magnet assembly. During testing, a strong coupled response at $i .62 \mathrm{~Hz}$ was observed and several "fixes" were attempted.

\section{TEST DESCRIPTION}

\section{Test Article}

The test article is shown schematically in Fig. 1 The girder is a prototype of the storage ring section 3 girder and the magnet is a Q5 $0.8-\mathrm{m}$ quadrupole which 

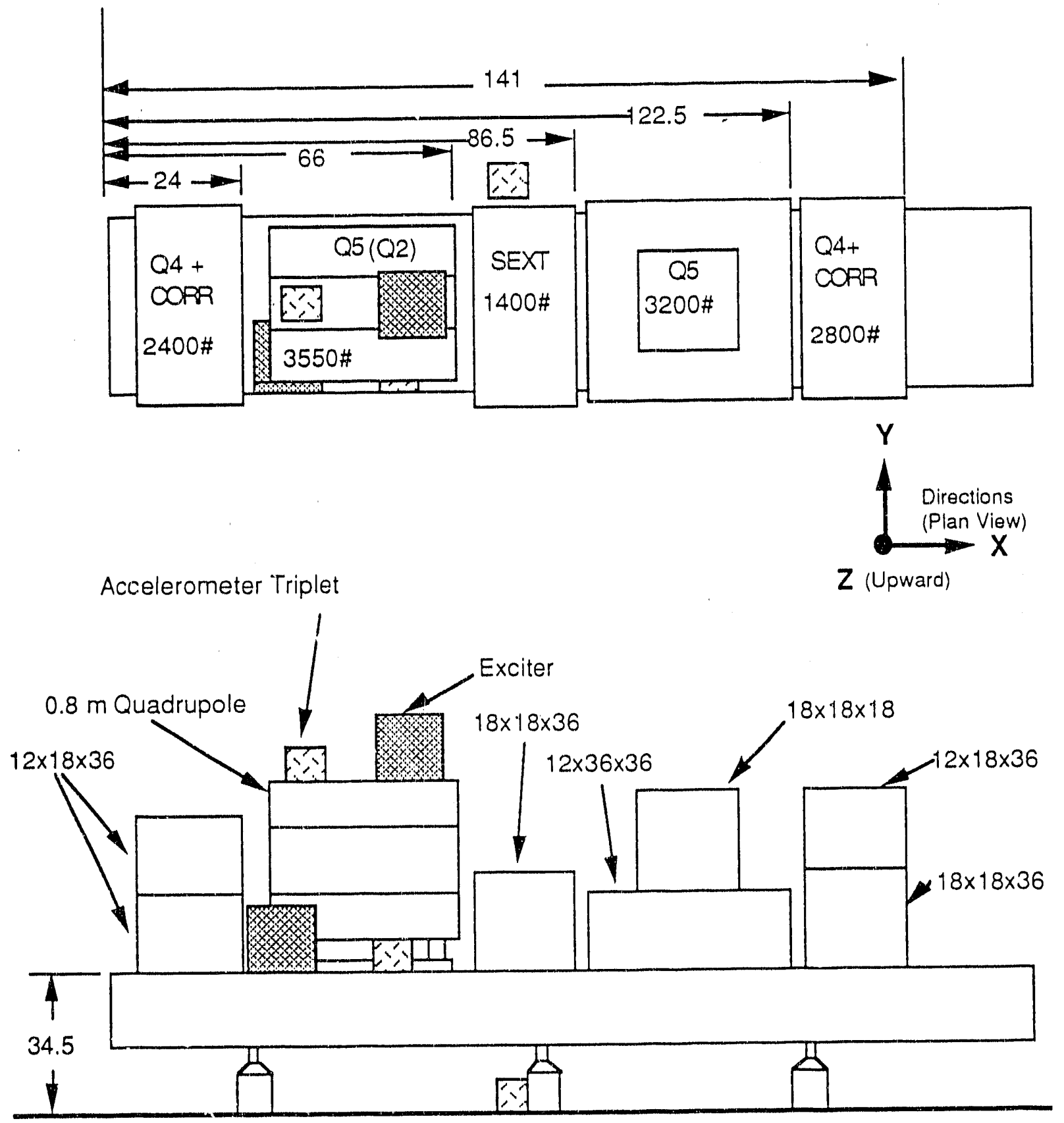

NOTE: All dimensions in inches

\section{Figure 1. Test Article}

\section{DISCLAIMER}

This report was prepared as an account of work sponsored by an agency of the United States This report was prepared as an account of work sponsored by anency thereof, nor any of their employees, makes any warranty, express or implied, or assumes any legal liability or responsibility for the accuracy, completeness, or usefulness of any information, apparatus, product, or process disclosed, or represents that its use would not infringe privately owned rights. Referprece herein to any specific commercial product, process, or service by trade name, trademark, manufacturer, or otherwise does not necessarily constitute or imply its endorsement, recommendation, of favoring by the United States Government or any agency thereof. The views and opinions of authors expressed herein wo noi nevessaitiy siaic or reflect those of the United States Government or any agency thereof. 
is similar to the Q2 used in the actual section 3 configuration. Girder jackscrew positions correspond to those designated as configuration $A$ in [1]. In the Phase I tests, the jackscrews are bolted to the Building 362 highbay floor. Exciter and accelerometer triplet (an array of three accelerometers oriented to measure acceleration in the three orthogonal directions) mounting locations are shown along with the concrete block locations, dimensions, and weights.

\section{Excitation Method}

Three force excitation methods were employed: (1) an electrodynamic exciter, used to produce a random and sinusoidial force, (2) hand impact, which was used to produce an impulse force, and (3) ambient floor motion. Tests were performed with the exciter mounted on the top of the magnet and on the girder near the location of the single magnet leg. Three nominal force levels, designated $0.6,0.4$, and 0.1 , were applied. For Phase I tests, the impulse force was applied to the magnet in both the $X$ and $Y$ directions at various locations near the top of the magnet. For the Phase II tests, the impulse force was applied at various locations as defined in the test matrix. Ambient floor motion was transmitted to the girder via the three jackscrew assemblies.

\section{Measurement Method}

Response was measured using accelerometer triplets mounted at various locations on the magnet, girder, and floor. The accelerometer signals were double integrated to obtain displacements in the range of $5.100 \mathrm{~Hz}$ and recorded on an FM tape recorder. They were analyzed on a HP $5451 \mathrm{C}$ Fast Fourier Spectrum Analyzer using a frequency range of $0-100 \mathrm{~Hz}$ with a bandwidth of $0.195 \mathrm{~Hz}$. The analysis process is discussed in Appendix A.

An accelerometer triplet mounted to the top of the magnet measured the magnet response, while another triplet was mounted on either the girder (near one leg of the two leg end) or on the floor near the center jackscrew and was used for response/transfer function measurements.

\section{Test Matrix}

A series of tests was planned and performed to accomplish the objectives of this study. The Phase I tests are defined as follows:

Test 1. Excitation: Randon force on top of magnet in three orthogonal directions with a frequency range of $0-150 \mathrm{~Hz}$, each at three nominal force levels.

Measurement/Objective: Displacement of magnet and girder in three orthogonal directions; used to determine system resonant frequencies. 
Test 2. Excitation: Random force on surface of girder in three orthogonal directions with a frequency range of $0-150 \mathrm{~Hz}$, each at three nominal force levels.

Measurement/Objective: Displacement of girder and top of magnet in three orthogonal directions. Calculate transfer functions between girder and top of magnet at specific frequencies.

Test 3. Excitation: Ambient floor motion.

Measurement/Objective: Displacement of floor and girder in three orthogonal directions. Calculate transfer functions between floor and girder at specific frequencies.

Test 4. Excitation: Sine force excitation on magnet

Measurement/Objective: Displacements which are used to determine mode shapes at specific frequencies.

Test 5. Excitation: Random force excitation on top of magnet in the $Y$ direction at a nominal level of 0.6. The channel iron which forms the base of the magnet is securely clamped to the base at both sides (double leg end) to increase the girder/magnet base contact force.

Measurement/Objective: Displacement of girder and magnet in three orthogonal directions. Calculate transfer function between girder and magnet at frequencies of interest.

Test 6. Excitation: Random force excitation on top of magnet in the $Y$ direction at nominal levels of $0.6,0.4$, and 0.1 . Wood blocks are jammed between the flange of the magnet and the girder on each side (double leg end) in an attempt. to reduce rotation of the magnet on its support system in the Y direction.

Measurement/Objective: Displacement of the girder and magnet in three orthogonal directions. Calculate transfer function between girder and magnet at frequencies of interest.

Test 7. Excitation: Impulse to top of magnet in the $\mathrm{X}$ and $\mathrm{Y}$ direction.

Measurement/Objective: Displacement of the girder and magnet in three orthogonal direstions. Calculate system frequencies and damping (percent of critical). 
The Phase II tests are defined as follows:

Test 8. Excitation: Impulse to the concrete blocks in the $\mathrm{X}$ and $\mathrm{Y}$ directions, applied symmetrically along the width and length of the girder, respectively. An impulse was also applied to the top and base of the single jackscrew in the $Y$ direction. With the exception of the quadrupole magnet, the test article is the same as previous tests.

Measurement/Objective: Displacement of the girder in three orthogonal directions. Calculate the system frequencies and damping (percent of critical).

Test 9. Excitation: Impulse to the concrete blocks in the $X$ and $Y$ directions, applied symmetrically along the width and length of the girder, respectively. The turnbuckles were loose for this test.

Measurement/Objective: Same as Test 8.

Test 10. Excitation and measurement/objective are the same as test 9; turnbuckles were loose and the bolts fastening the jackscrew pedestals to the floor were loose.

Test 11. Excitation and measurement/objective are the same as Test 9; the two outer bolts on each pedestal were tightened.

Test 12. Excitation and measurement/objective was the same as Test 9; all bolts and the turnbuckles were tight.

Test 13/15. Excitation: Ambient floor motion applied to all three jackscrew locations. Similar to Test 3, except the quadrupole is removed. Test 15 was conducted after Test 14, to verify the effects of the tightness of the jackscrew pedestal bolts.

orthogonal directions.

Measurement/Objective: Displacement of the girder in three

Test 14. Excitation and measurement/objective are the same as that of Test 13; the turnbuckles and jackscrew pedestal bolts were loose.'

\section{RESULTS}

The results for the phase one tests are as follows:

Test 1. The major system frequencies, for the three orthogonal excitation directions, determined from the power spectral density plots of Appendix $B$, are tabulated in Table 1. There are six frequencies ranging from $7.62 \mathrm{~Hz}$, which is 
Table 1. System Response Frequencies for Test 1

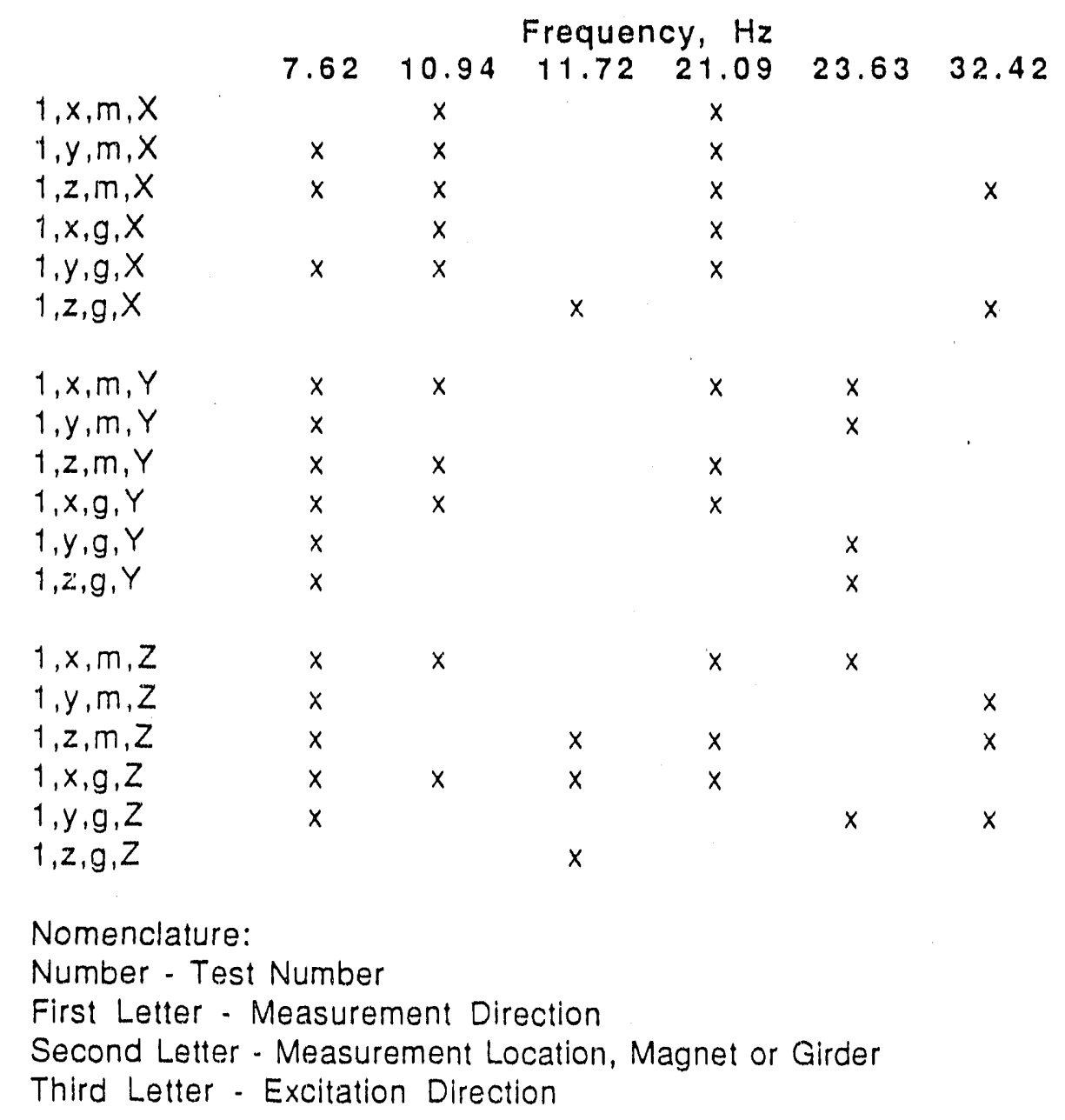


present for almost all conditions, to $32.42 \mathrm{~Hz}$, with primary response in the $\mathrm{Z}$ direction. RMS displacement amplitudes, which are also determined from the power spectral density plots are given in Appendix C. A maximum magnet displacement (wideband) of 3.29 microns rms is measured for an excitation of 0.6 in the $Y$ direction. It should be noted that the transfer functions, which are the output amplitude divided by the input amplitude, are less than one for $\mathrm{X}$ and $\mathrm{Y}$ at $7.62 \mathrm{~Hz}$ due to the exciter location.

Test 2. The main system frequencies, which are also obtained from the power spectral density plots of Appendix B, are shown in Table 2. As expected, the frequency content is very similar to that of Test 1; the slight variations are due to analysis rather than actual system response. The largest observed displacement amplitude (Appendix C) is 1.47 microns rms at a frequency of $7.62 \mathrm{~Hz}$. The transfer function relating the girder and magnet response in the $Y$ direction is 1.8. It should be noted that the transfer function corresponding to a particular mode is a function of the measurement locations. For the expected mode shape, it would take on a much larger value if one were to provide excitation at a lower position closer to the floor. This can be seen in the ambient displacement response in the $Y$ direction (Table 4); also see comments on magnification factor in Test 7 results below.

Test 3. The main system frequency, $7.62 \mathrm{~Hz}$, can be seen in Table 3 . Other frequencies are present, but the large amplitude of the $7.62 \mathrm{~Hz}$ displacement predominates the response, particularly in the $Y$ direction. Table 4 shows the total rms displacement on the magnet as a result of ambient floor motion while the $7.62 \mathrm{~Hz}$ contribution is shown in Table 5. The $7.62 \mathrm{~Hz}$ displacement contributes $32 \%, 95 \%$, and $32 \%$ to the total magnet displacement in the $\mathrm{X}, \mathrm{Y}$, and Z directions, respectively. The transfer function concept has to be used with caution in this situation; because of the large mass of the test article, energy may drive the floor at $7.62 \mathrm{~Hz}$ which, would result in an abnormally low transfer functior.

Test 4. Mode shapes at frequencies of $7.62,10.94,11.72,21.09$, and $23.63 \mathrm{~Hz}$ were determined from single frequency sine excitation in the $\mathrm{X}, \mathrm{Y}$, and $\mathrm{Z}$ directions. The mode shapes at 7.62 and $10.94 \mathrm{~Hz}$ are sketched in Figs. $2 \mathrm{a}$ and $2 \mathrm{~b}$, respectively. Due to translational force coupling, the three higher mode shapes are not as easily defined as the two lower ones.

Test 5. The main system frequencies, given in Table 3, agree with those of Test 1. with the exception of some higher ones. The omissions are of an analytical nature, as the test objective was to observe the response at $7.62 \mathrm{~Hz}$ when the magnet base was securely clamped to the girder. The maximum wideband displacement amplitude measured in the $Y$ direction was 5.27 microns rms, which was 1.6 times the Test 1 value. Most of the increased contribution was from higher modes. Clamping the base to the girder to increase mounting stiffness had minimal effect on the $7.62 \mathrm{~Hz}$ system response frequency. 

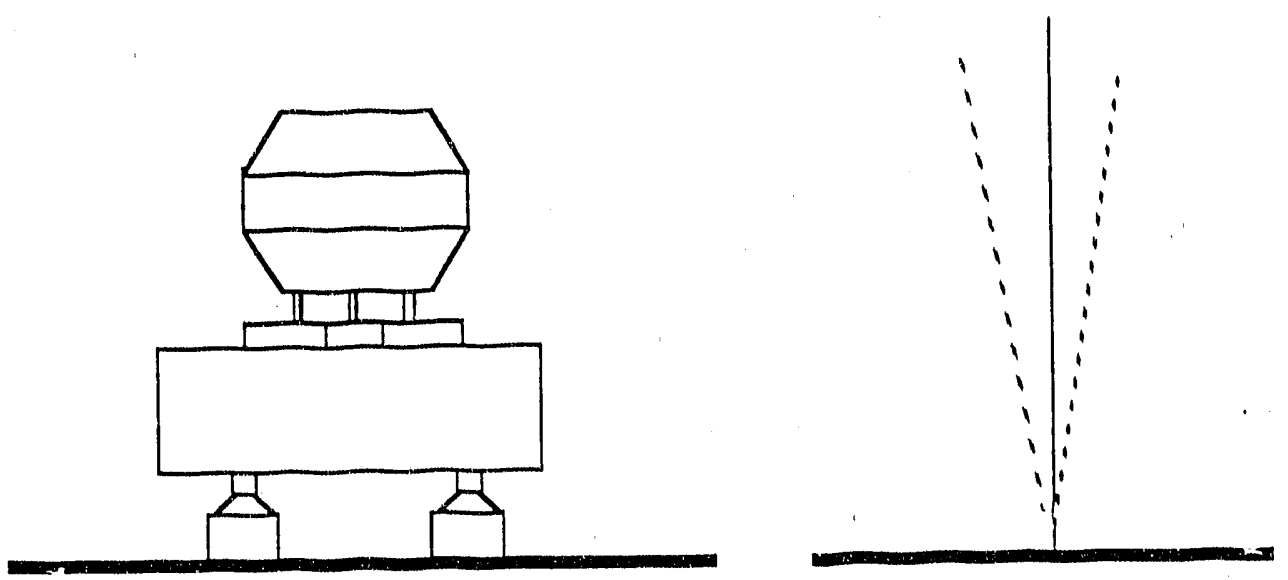

Figure 2a. $7.62 \mathrm{~Hz}$ Mode Shape
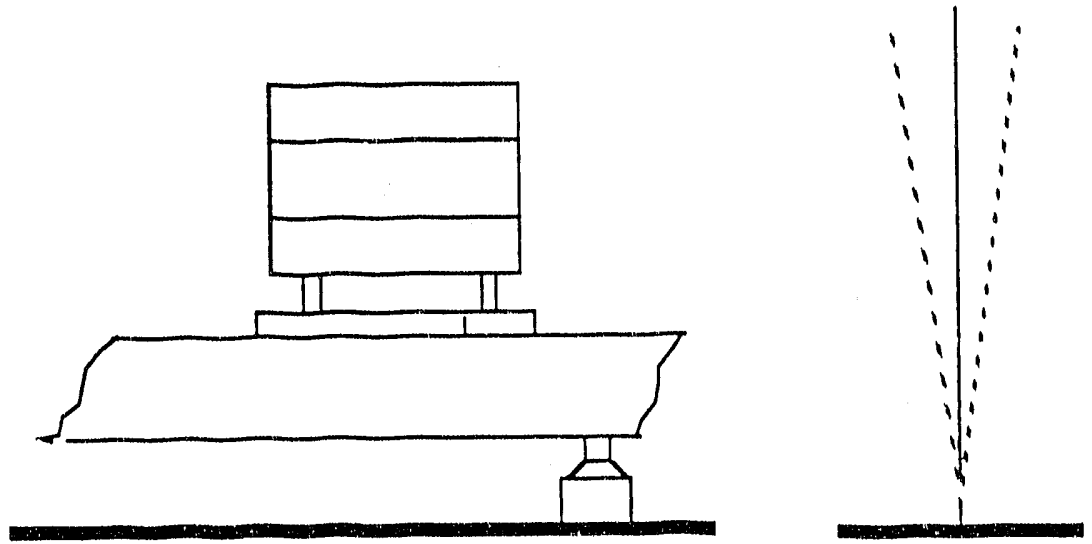

Figure 2b. $10.94 \mathrm{~Hz}$ Mode Shape 
Table 2. System Response Frequencies for Test 2

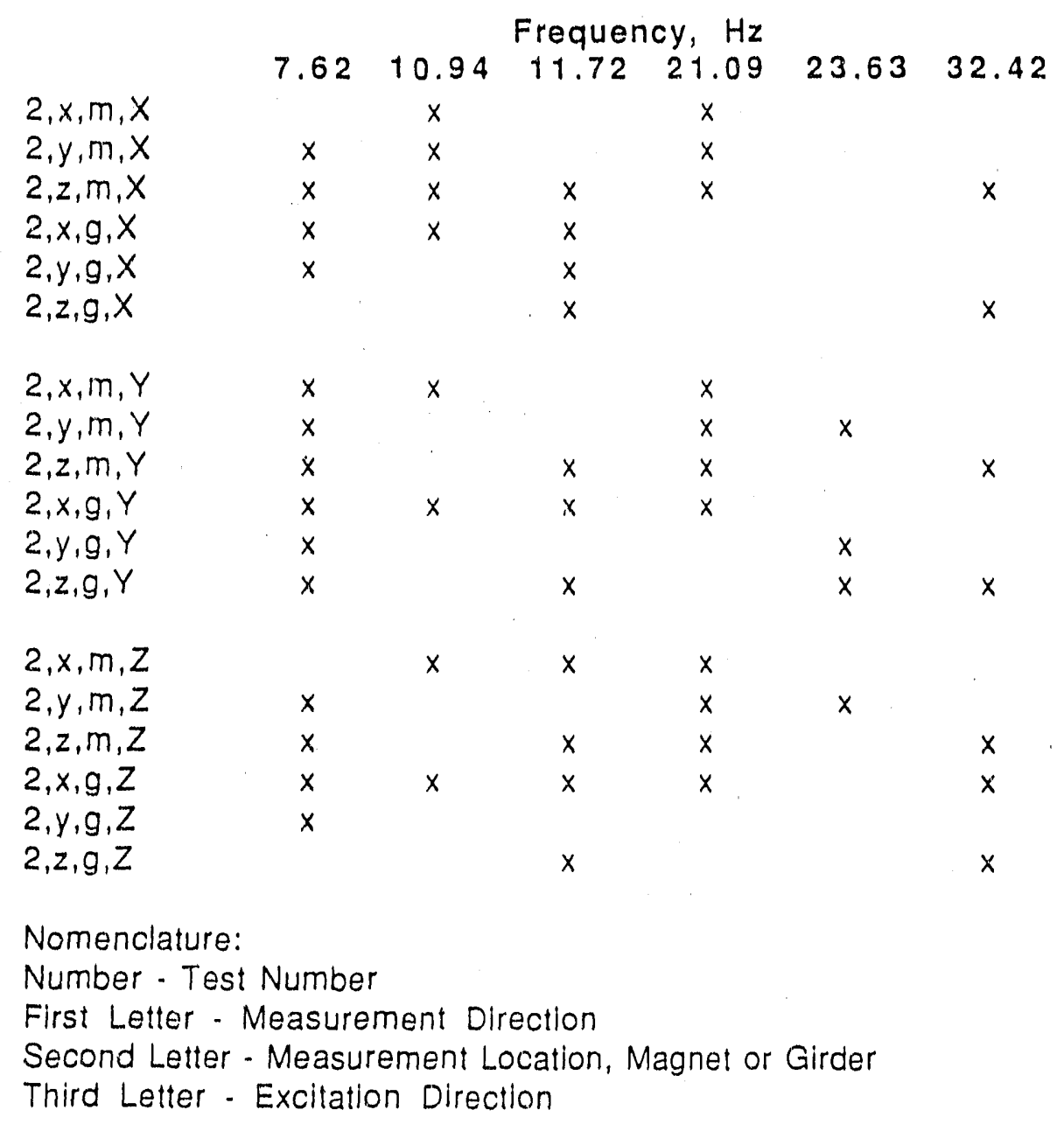


Table 3. System Frequencies for Tests 3, 5, and 6

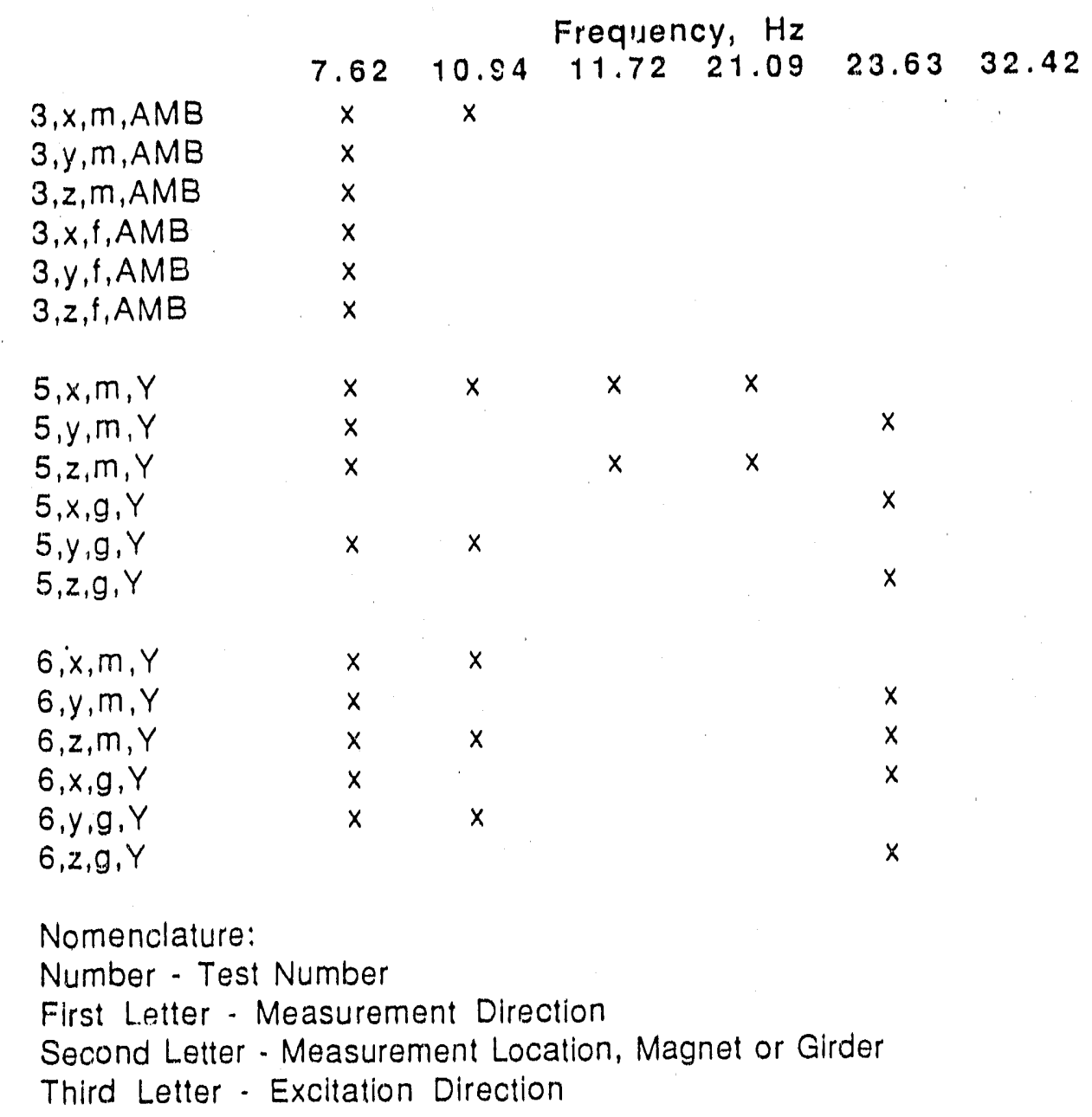


Table 4. Displacement Response for Ambient Excitation (Wideband, Test 3)

$\begin{array}{cc}\text { Measurement Position Displacement, microns, rms } \\ \\ \text { X,Magnet } & 0.114 \\ \text { Y,Magnet } & 0.526 \\ \text { Z,Magnet } & 0.0871 \\ \text { X,Floor } & \\ \text { Y,Floor } & 0.0711 \\ \text { Z,Floor } & 0.0529 \\ \end{array}$

Table 5. Displacement Response for Ambient Excitation $(7.62 \mathrm{~Hz}$, Test 3 )

Measurement Position Displacement,microns,rms

$\begin{array}{lc}\text { X,Magnet } & 0.0368 \\ \text { Y,Magnet } & 0.5 \\ \text { Z,Magnet } & 0.0282 \\ & \\ \text { X,Floor } & 0.0428 \\ \text { Y,Floor } & 0.0392 \\ \text { Z,Floor } & 0.000428\end{array}$


Test 6. The system response is approximately the same as that of Test 5 ; the frequencies, given in. Table 3 , are the same, with the maximum displacement contribution again from $7.62 \mathrm{~Hz}$ mode. The total wideband displacement amplitude in the $\mathrm{Y}$ direction is 1.16 times the Test 1 value. However, the $7.62 \mathrm{~Hz}$ displacement contribution is 1.1 times that of Test 1 . It appears that the wood blocks placed between the girder and magnet flange are of no consequence in reducing the $7.62 \mathrm{~Hz}$ response.

Test 7. An impulse force is applied to either the magnet or the girder and the resulting response is recorded and analyzed. The resultant responses are shown in Appendix D, and summarized in Table 6. The 7.62 and $10.94 \mathrm{~Hz}$ frequencies are observed on all tests; the 11.72 and $21.09 \mathrm{~Hz}$ frequencies are related to excitation in the $\mathrm{X}$ direction. Of primary concern is the measured damping value of 0.00892 in the $X$ direction and an average value of 0.00716 in the $\mathrm{Y}$ direction. If the system is simplified to assume a single degree of freedom system, the magnification factor is equal to the reciprocal of two times the damping value. For the $\mathrm{X}$ direction damping value of 0.00892 the magnification factor is 56; for the $Y$ direction the magnification factor is 69.8. The magnification factors are considered much too large for the required stability necessary of the magnet/girder system.

The Phase II tests are as follows (displacement and frequency plots are given in Appendix E):

Test 8. The test article is the same as for Test 7 with the exception of the quadrupole magnet. This test is used to deter.nine system frequencies and damping with the quadrupole magnet removed. Excitation force was applied in the $\mathrm{X}$ and $\mathrm{Y}$ directions at locations on the concrete blocks which were chosen to minimize torsional response. Two additional locations chosen were on the jackscrew top and jackscrew pedestal, with excitation only in the Y direction. The principal frequency in the $\mathrm{X}$ direction increased to $12.5 \mathrm{~Hz}$ and the $\mathrm{Y}$ principal frequency increased to $9.4 \mathrm{~Hz}$ (see Table 8). These values are reasonable even if one only considers the decrease in mass of the system. The measured damping values (see Table 9) are consistent with those of Test 7 . Similar system response was observed with excitation applied to the jackscrew and jackscrew pedestal.

Test 9. The turnbuckles were loosened for this test. No appreciable change was observed between this test and Test 8 , see Tables 8 and 9 .

Test 10. The turnbuckles remained loose and all of the bolts anchoring the pedestals to the floor were removed. When the bolts were removed, the outer two bolts on each pedestal were found to be considerably tighter than the two inner ones. The measured frequencies in the $\mathrm{X}$ and $\mathrm{Y}$ direction lowered to $8.4 \mathrm{~Hz}$ and $5.9 \mathrm{~Hz}$ respectively. While the damping in the $\mathrm{X}$ direction increased to 0.0101 , an increase of 1.42 times, the $Y$ direction damping increased to 0.0344 , an increase of 4.74 times. 
Table 6. Impulse Excitation Response for Test 7

\begin{tabular}{|c|c|c|c|c|c|c|c|}
\hline $\begin{array}{c}\text { Exc. } \\
\text { Direction }\end{array}$ & $\begin{array}{l}\text { Meas. } \\
\text { Direction }\end{array}$ & & & Freque & ncies & & Damping \\
\hline$x$ & X,Magnet & 7.62 & 10.94 & 11.72 & 21.09 & & 0.00892 \\
\hline$x$ & Y,Magnet & 7.62 & 10.94 & 11.72 & 21.09 & 23.40 & \\
\hline$x$ & X,Girder & 7.62 & 10.94 & & 21.09 & 23.63 & \\
\hline$x$ & Y,Girder & 7.62 & 10.94 & 11.72 & 21.09 & & \\
\hline$Y$ & X Magnet & 7.62 & 10.94 & & 21.09 & 23.63 & \\
\hline$Y$ & Y,Magnet & 7.62 & 10.90 & & & & 0.00647 \\
\hline$Y$ & X,Girder & 7.62 & 10.94 & & & & 0.00708 \\
\hline$Y$ & Y,Girder & 7.50 & 10.94 & & & & 0.00794 \\
\hline
\end{tabular}

Table 7. Principal System Frequencies for Phase I Tests

$7.62 \mathrm{~Hz}$ - Magnet / Girder response in the $Y$ direction

$10.94 \mathrm{~Hz}$ - Magnet/Girder response in the $X$ direction

$11.72 \mathrm{~Hz}$ - - Coupled response, strong in the $X$ and $Z$ directions

$21.09 \mathrm{~Hz}$ - - Higher mode of MagnevGirder in the $Y$ direction

$23.63 \mathrm{~Hz}$ - - Higher mode of Magnet/Girder in the $Y$ direction

$32.42 \mathrm{~Hz}$ - . $\mathrm{Z}$ direction response 
Table 8. Frequency Response for Phase II Tests

Test \#

8

8

8

8

9

9

10

10

11

11

\section{ExcitationLocation}

$Y$, On block, center of girder

$X$, On block, center of girder

$Y$, On top of single jackscrew

$Y$, On base of single jackscrew

$Y$, On block, center of girder

$X$, On block, center of girder

$Y$, On block, center of girder

$X$, On block, center of ('irder

$Y$, On block, center of girder

$X$, On block, center of girder

$Y, O n$ block, center of girder

$X$, On block, center of girder

$Y$, On block, center of girder

$X$, On block, center of girder
$X$ Direction $Y$ Direstion

9.15

12.5

9.3

9.3

9

12.1

5.9

8.4

7.9

11.2

$11.7 / 12.4$

9.2

9.25

9.25

Table 9. Damping for Phase II Tests

Test \#

8

8

8

8

9

9

10

10

11

11

12

12

13

13

\section{Excitation Location}

$Y$, On block, center of girder

$X$, On block, center of girder

$Y$, On top of single jackscrew

$Y$, On base of single jackscrew

$Y$, On block, center of girder

$X$, On block, center of girder

$Y$, On block, center of girder

$X$, On block, center of girder

$Y$, On block, center of girder

$X$, On block, center of girder

$Y$, On block, center of girder

$X$, On block, center of girder

$Y$, On block, center of girder

$X$, On block, center of girder
$X$ Direction $Y$ Direction

0.00713

0.00724

0.00587

0.00967

0.00622

0.00735

0.0344

0.0101

0.00858

0.00769

0.00726

0.00774

0.00775

0.00678 
Test 11 . The two outer bolts on each pedestal were retightened. When the bolts were tightened, the gap between the pedestal lower plate and the floor decreased considerably, indicating a lateral load to the jackscrew assembly was changing. The frequencies and damping observed are consistent with Tests 7-9.

Test 12. The remaining inner bolts on each pedestal were tightened. The fiequencies are again consistent with Tests 7-9 and Test 11.

Test 13. The turnbuckles were tightened; test conditions are now identical to Test 8 . Frequency and damping are also comparable to Test 8 . Also, ambient floor motion was used to excite the assembly. Measured displacements, resulting from ambient floor excitation, are listed in Appendix $E$.

Test 14. In this test, the turnbuckles were loose and the bolts anchoring the pedestals to the floor were loosened; ambient floor motion was used to excite the assembly. A summary of the displacement response change is shown in Table 10. The values shown are the ratio of system response to ambient floor excitation with the pedestal bolts loose (Test 14) to the average of the two tests with the bolts tight (Tests 13 and 15). The wideband ratios are lower for the $X$ and $Y$ directions but somewhat higher in the $\mathrm{Z}$ direction. However, at the $9.37 \mathrm{~Hz}$ frequency, which is the principal magnet/girder response in the $\mathrm{Y}$ direction, the $\mathrm{X}$ direction response increases by a factor of 6.7 , while the $Y$ direction response decreases by a factor of 0.263 .

Table 10. Change in Ambient Response (Test 14 Values Divided by the Average of Test 13 and 15 Values)

Direction

$\begin{array}{cccccc} & \text { Wideband } & 9.37 \mathrm{~Hz} & 11.5 \mathrm{~Hz} & 12.9 \mathrm{~Hz} & 13.5 \mathrm{~Hz} \\ X & 0.761 & 6.7 & 0.327 & 0.0328 & 0.0468 \\ Y & 0.367 & 0.263 & 0.363 & 0.058 & 0.12 \\ Z & 2.48 & 1.55 & 1.03 & 0.212 & 0.329\end{array}$

Test 15. The turnbuckles and pedestal bolts were retightened and the response to ambient floor motion measured. This test is similar to Test 13 , being used to verify the system response change with the pedestal anchoring. Considering the random nature of the ambient floor excitation the measured displacements from each test compare well. 


\section{DISCUSSION}

\section{Phase I Tests}

\section{Frequencies}

There are six response frequencies observed in the power spectral density plots of Appendix B. These are listed in Tables 1-3. From this and Test 4, one can determine the principal system response to each frequency. The results are summarized in Table 7.

The $7.62 \mathrm{~Hz}$ response is a coupled rigid body mode of the magnet/girder assembly in the $\mathrm{Y}$ direction and the corresponding large displacements tend to dominate the system displacement. The $10.94 \mathrm{~Hz}$ res, unse is a similar rigid body response in the $\mathrm{X}$ direction; displacement amplitudes associated with it are approximately one-half that of the $7.62 \mathrm{~Hz}$ response. The $11.72 \mathrm{~Hz}$ response is strong in the $\mathrm{Y}$ and $\mathrm{Z}$ directions. Frequencies of $21.09 \mathrm{~Hz}$ and $23.63 \mathrm{~Hz}$ are complex modes of the magnet/girder assembly. The $32.42 \mathrm{~Hz}$ response is associated with displacement in the $\mathrm{Z}$ direction.

\section{RMS Displacements}

It is significant to note that the RMS displacements associated with force excitation are large relative to the corresponding measured displacements from the tests of Ref. 1 in which the magnets were simulated by concrete shielding blocks. This, of course, can be attributed to the lower damping of the magnet support assemblies exhibited in the subject tests. Wideband RMS displacement of the quadrupole in response to ambient floor motion exceeded 0.5 microns RMS in the $\mathrm{Y}$ direction. It remains to be determined whether or not amplitudes of this magnitude, with the dominant contribution at low frequencies, will exceed the dynamic range of the feedback control system.

\section{Transfer Functions}

The transfer functions calculated in this study are dependent on the excitation position with respect to the mode shape. Translational motion between the three axes also complicates mode shape determination, particularly at the higher frequencies. In using the transfer function, consideration should be given to excitation location relative to the mode shape, and displacement amplitude. A more meaningful representation of magnification factor would be to base it on measured damping, calculating magnification factor as $1 /(2 \xi)$, where $\xi$ is the damping factor. With this representation, at $7.62 \mathrm{~Hz}$ magnification factors of 56 and 69.8 in the $\mathrm{X}$ and $\mathrm{Y}$ directions, respectively, are obtained. 


\section{Mode Shapes}

The displacements at $7.62 \mathrm{~Hz}$ and $10.94 \mathrm{~Hz}$ are the largest and their mode shapes are well defined. Consequently, the 7.62 and $10.94 \mathrm{~Hz}$ nıdes will be the only ones described. The mode shape for the $7.62 \mathrm{~Hz}$ mode is shown in Fig. 2a and the $10.94 \mathrm{~Hz}$ mode shape is shown in Fig. 2b. Due to translational motion between the various axis, the higher frequency modes are not well defined.

\section{Phase II Tests}

In this series of tests, we have the opportunity to study the girder response uncoupled from the magnet. We can also assess why the girder response is so much different than that of Ref 1 . With the exception of Tests 10 and 14, the measured frequency and damping agree with what would be expected from Phase I tests. However, Tests 10 and 14 shnw the response variation which may be expected if the jackscrews lateral loads are changed, such as, by tightening the pedestal anchor bolts.

\section{CONCLUSION}

\section{Phase I Tests}

A strong, coupled magnet/girder mode response occurs at a frequency of $7.62 \mathrm{~Hz}$ resulting in very large magnification factors (low damping values) and large displacements. It appears that a low frequency rigid body mode of the magnet coincides with a girder mode frequency. Figure 3, an unpublished plot from the Ref. 1 study, which is a plot of an impulse response of the girder in the $Y$ direction under similar conditions to that of this study, shows system responses at $7.5,9.5$, and $13.5 \mathrm{~Hz}$. It is probable that the $7.5 \mathrm{~Hz}$ frequency shown is responsible for the girder response.

Even though the $7.62 \mathrm{~Hz}$ displacement is within the frequency range of the control system, the concern is that the magnitude of displacement may exceed the dynamic range of the feedback control system. Since only one magnet was mounted, with the others represented by concrete blocks, other magnets mounted to the girder could considerably aggravate the situation, leading to even larger transfer functions and motions.

\section{Phase II Tests}

The Phase II tests show the effect of jackscrew conditions on system response. When the pedestal bolts were loose, the jackscrew/pedestal assembly deflected slightly from its initial vertical position, resulting in a shift in position of the jackshaft within the screw assembly. In this test (Test 10), the result was a significant reduction of frequency and a large increase in damping. This 


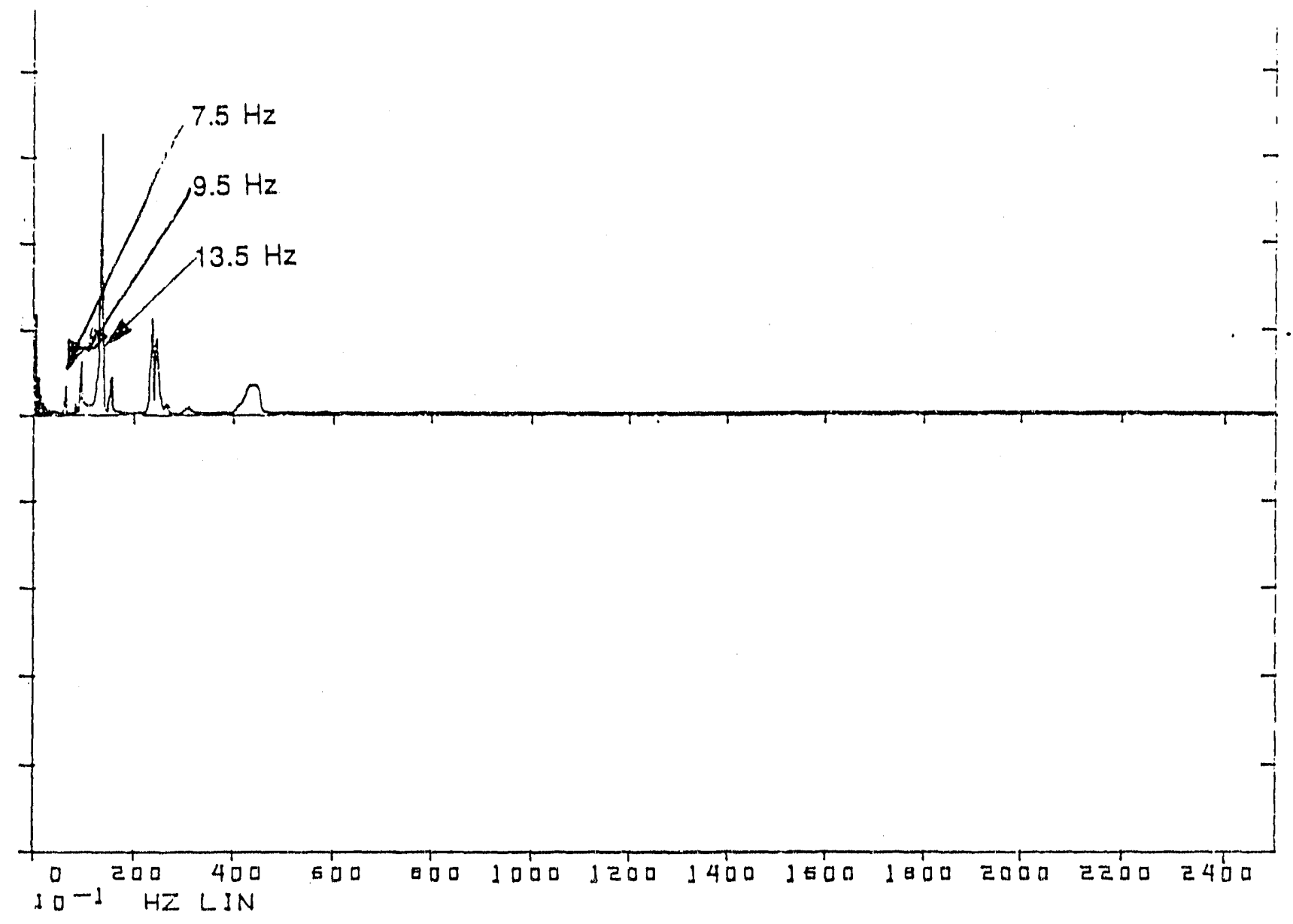

FIGURE 3. Impulse Excitation, Test APSC $+11 / 2$ inches [1] 
increase in damping resulted in a magnification factor (calculated from the damping value) in the $\mathrm{Y}$ direction of 14.5 , a significant reduction from the initial value of 69.8. However, the $\mathrm{X}$ direction ambient response increased by a factor of 6.7 in the $9.37 \mathrm{~Hz}$ mode. If the jackshaft/screw assembly was initially misaligned, it would be possible, by adjustment of the horizontal positioning screws on the top of the jackscrew, to change aligrment, resulting in a change in system response. Other factors, such as initial position during fabrication, floor flatness, stresses set up during welding, and tighiness of tha pedestal/floor anchoring bolts could also effect the alignment, resulting in unpredictable system response.

The main concern with the girder/jackscrew assembly is the random system response. For some conditions, such as in [1] the response was acceptable, but for this study the response was unacceptable.

\section{RECOMMENDATIONS}

Due to its basic construction, the jackscrew assemblies vibration response cannot be accurately predicted. It would be best, from a vibration response standpoint, to eliminate them from the structural support system. A large massive concrete support system would have the following advantages: 1) A larger amount of energy would be required to excite the large mass. A common practice in the design of low vibration systems is to have as large a mass as is practical. 2) There would be fewer vibration frequencies, both rigid body and bending, and consequently a lesser chance for a resonant frequency of the magnet/beam line assembly to ccincide and/or couple with a base/girder resonant frequency. 3) Damping is typically high in systems incorporating large concrete structures resulting in lower magnification factors. 4) A much more predictable response, which, if any coupled response between the magnet/magnet support system would orcur, would facilitate making design changes to eliminate the problem. However, to achieve the above advantages, it will be necessary to rigidly anchor the massive concrete structure to the floor. Any flexibility between the concrete structure and the floor could introduce a low frequency resonant response to the system.

\section{FUTURE STUDIES}

Assuming a concrete/steel structure is designed to support the magnet/beamline, it should be tested to determine its vibration characteristics and to verify acceptable performance.

The magnet support structures incorporate leveling and adjustment devices which are similar to the girder jackscrew assemblies. The vibration characteristics of the magnets and their support systems should also be 
measured and evaluatrid. An additional consideration is the system response to magnet cooling water which needs to be investigated.

All equipment which mechanically relates to the beamline/storage ring should be considered from a vibration standpoint to eliminate any "surprises." As they become available, each complete beamline section configuration should be tested, along with the dynamic characteristics of the storage ring floor.

\section{REFERENCES}

[1] Wambsganss, M. W., Jendrzejczyk, J. A., and Chen, S. S., "Vibration Study of the APS Magnet Support Assemblies," APS/IN/VIB/90-3, November 1990

[2] Jendrzejczyk, J. A., Wambsganss, M. W., and Smith, R. K., "Vibration Study of the APS Vacuum-Chamber/Girder Assembly," APS/IN/VIB/91-1, February 1991 


\section{Apperidix A}

The Hewlett-Packard 5451C Analysis Process 


\section{The Hewlett-Packard 5451C Analysis Process}

This Appendix presents an overview of the analysis process used to reduce the displacement data for this study. A data block size of 1024 was used with a frequency range of $0-100 \mathrm{~Hz}$. The filter bandwidth is 100 divided by 512 , or $0.1953 \mathrm{~Hz}$ per channel. For the actual study, a total average time of 51.2 seconds was used. A maximum of four channels with the same bandwidth may be simultaneously analyzed.

A $40 \mathrm{~Hz} 1$ volt RMS input signal, Figure A1, is used as an input. A Fourier Transform is generated, the real part shown in Figure A2 and the imaginary part in Figure A3. This information is used to compute a Power spectral Density Function, shown in Figure A4. It is integrated, Figure A5, to obtain the area under the curve which is the mean square voltage. Since the Fourier Transform is a symmetrical function, only the positive portion is shown in the figures, therefore to obtain the proper rms voltage, the mean square value must multiplied by 2. The printout of the last channel (511) of Figure A5 is 0.4888 volts squared. This is equivalent to an $\mathrm{rms}$ voltage of 0.988 volts rms. 


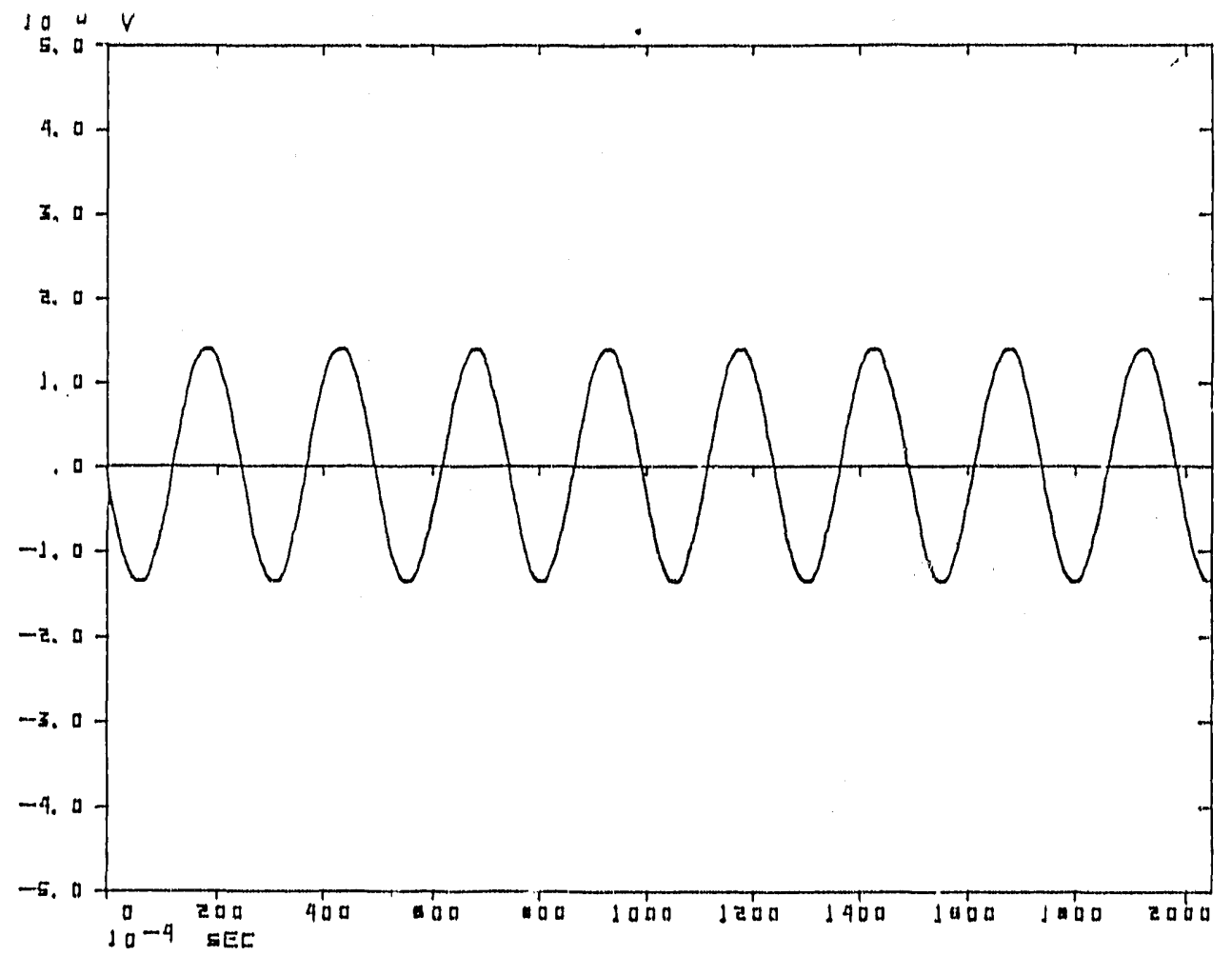

FIGURE A1, Inupt signal, Sinewave at $40 \mathrm{~Hz}$ 


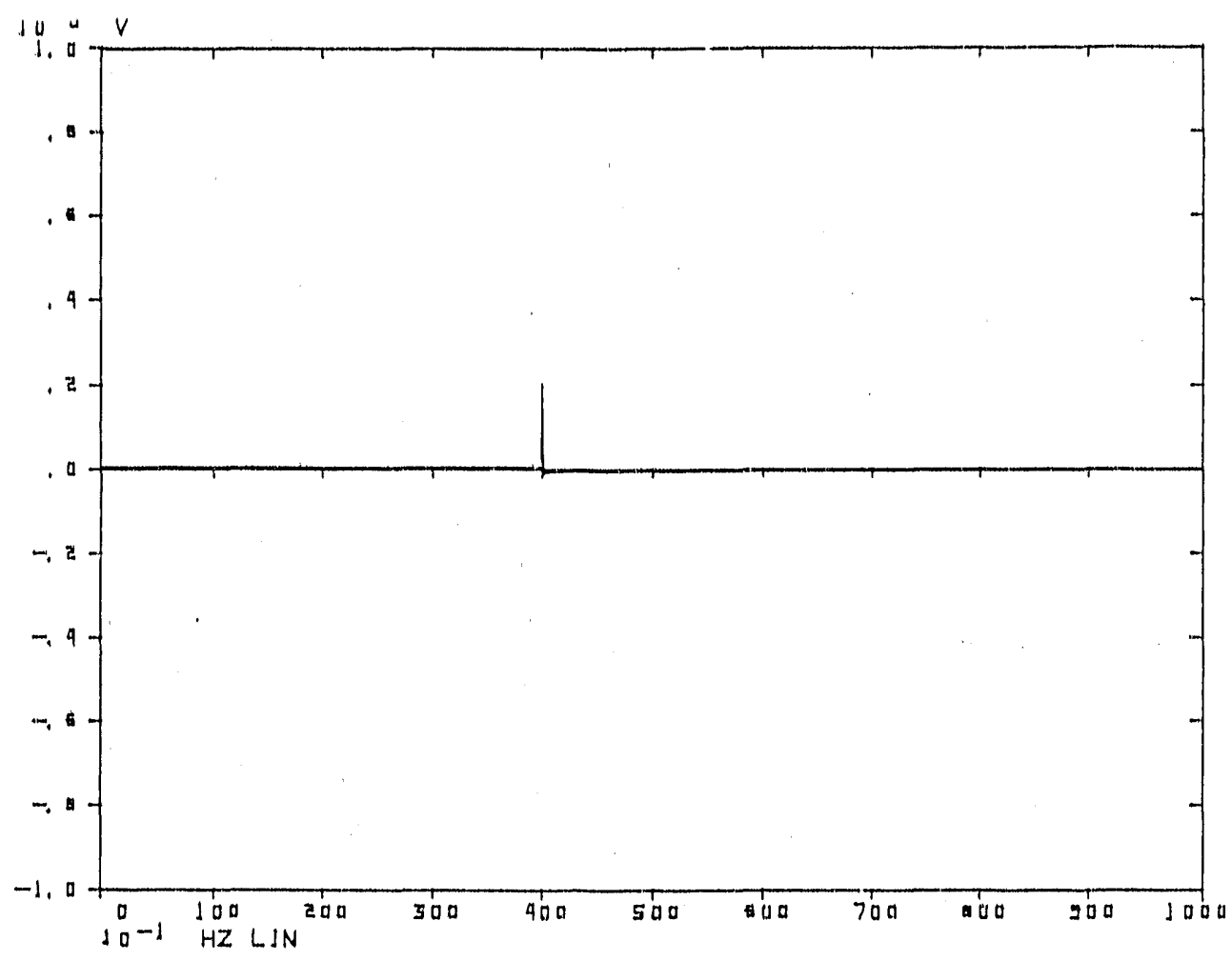

FIGURE A2. Real part of Fourier Transform

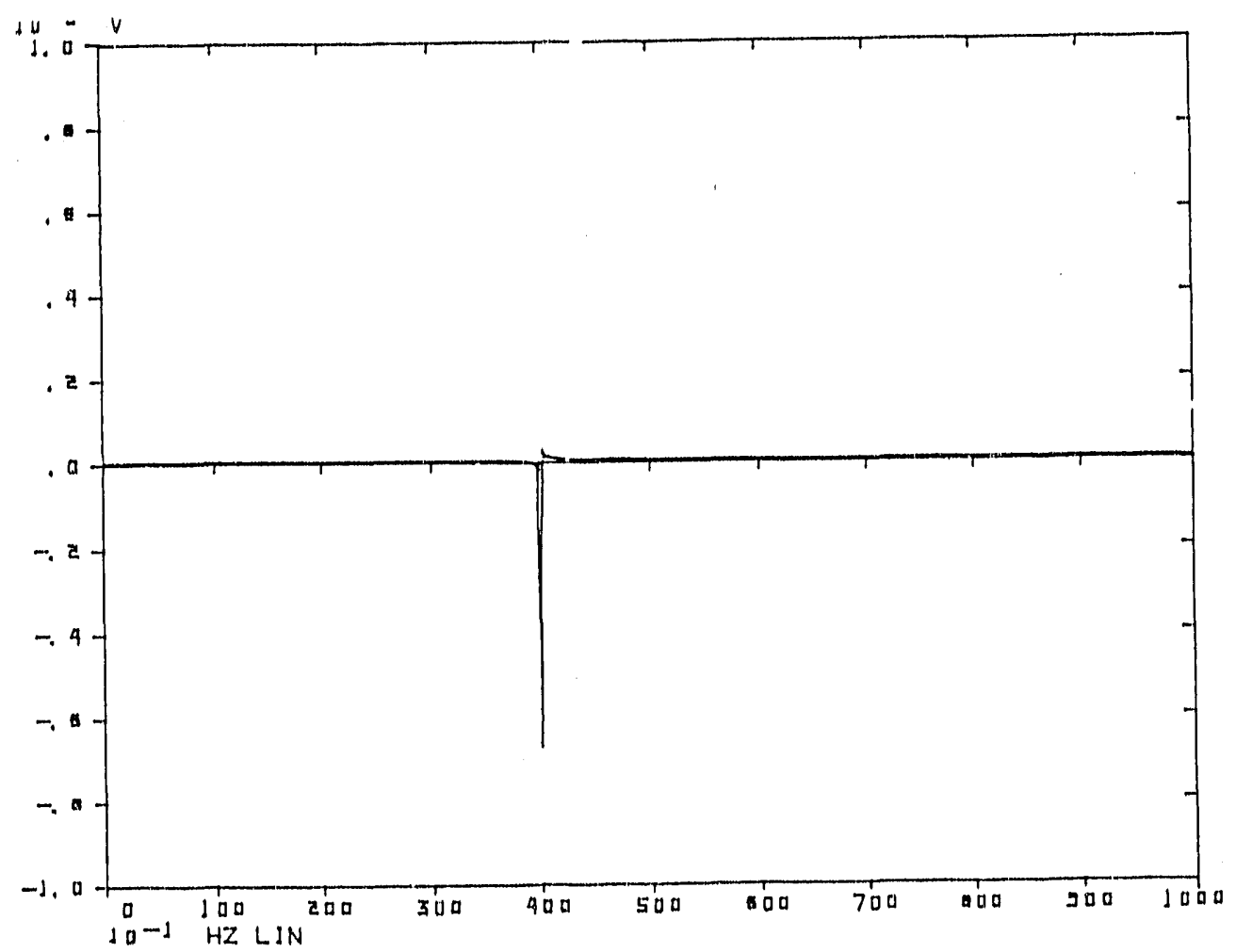

FIGURE A3. Imaginary part of Fourier Transform 


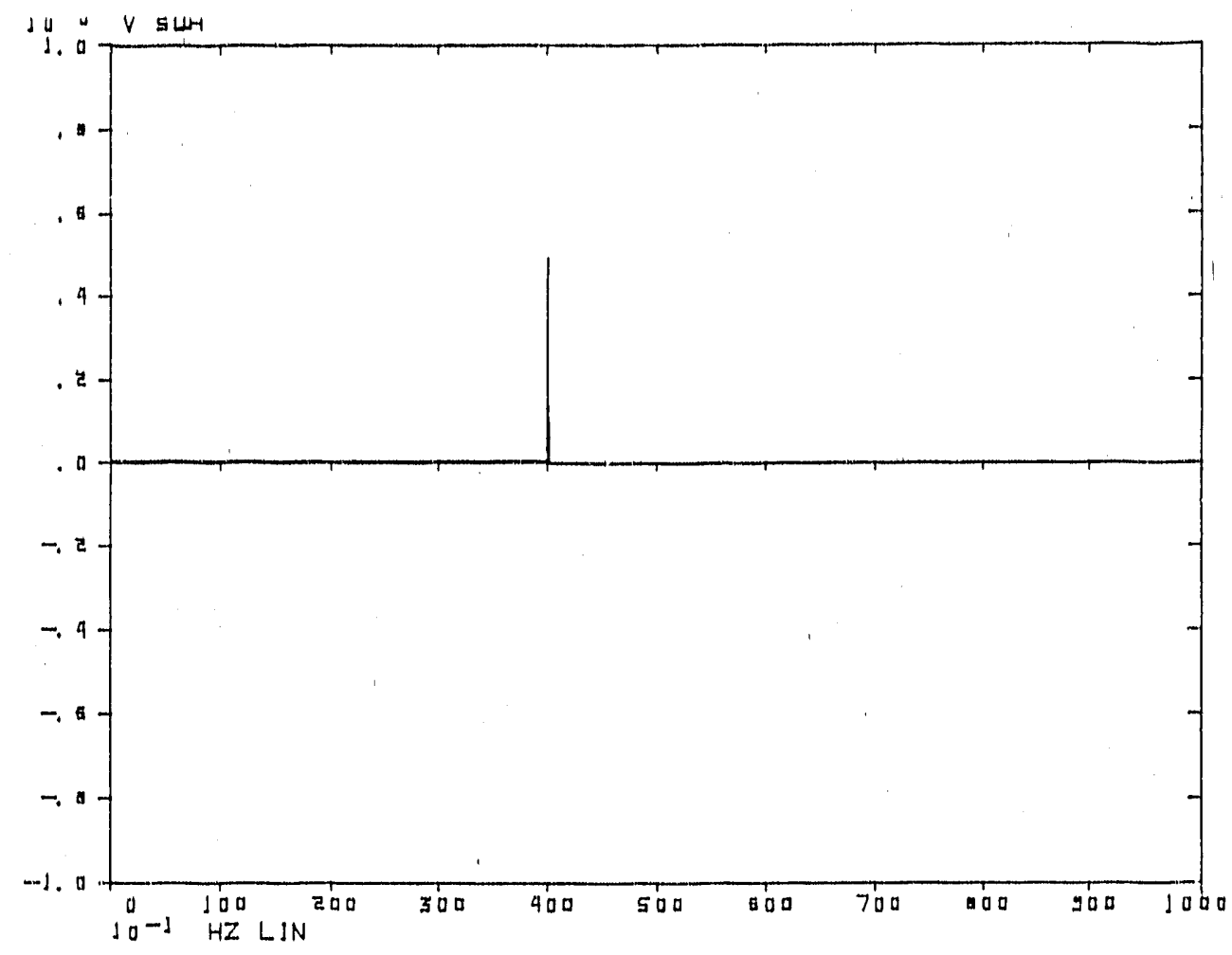

FIGURE A4. Power Spectral Density

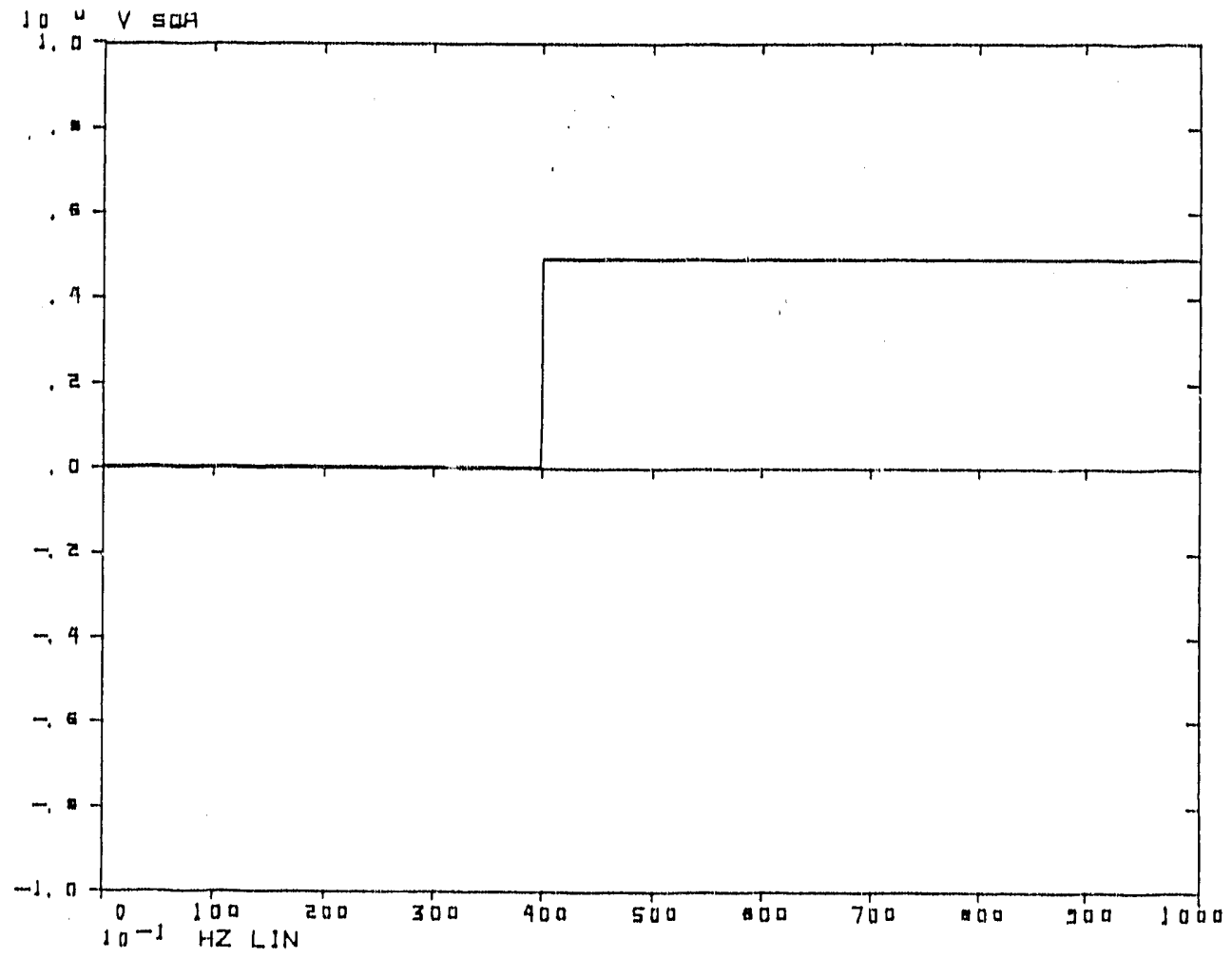

FIGURE A5. Integral of Power Spectral Density 
Appendix B

Power Spectral Density Plots for Phase I Tests 


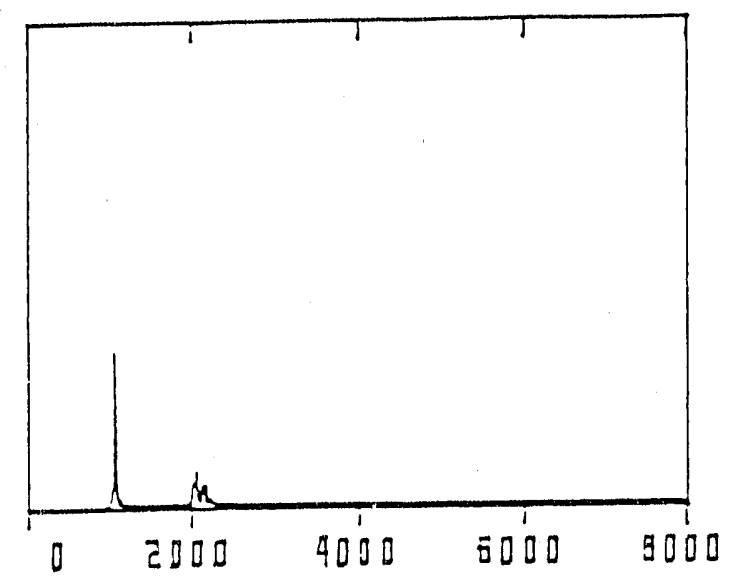

$10^{-E} H Z L I N$

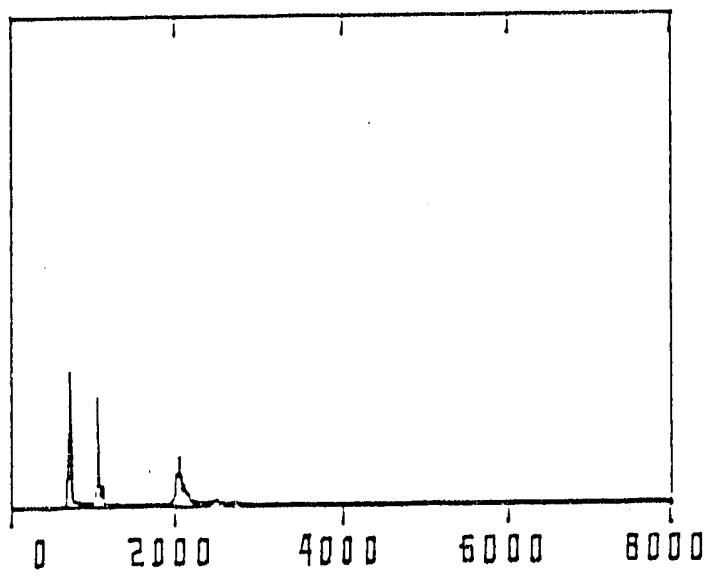

$10^{-2} \quad H Z L I N$

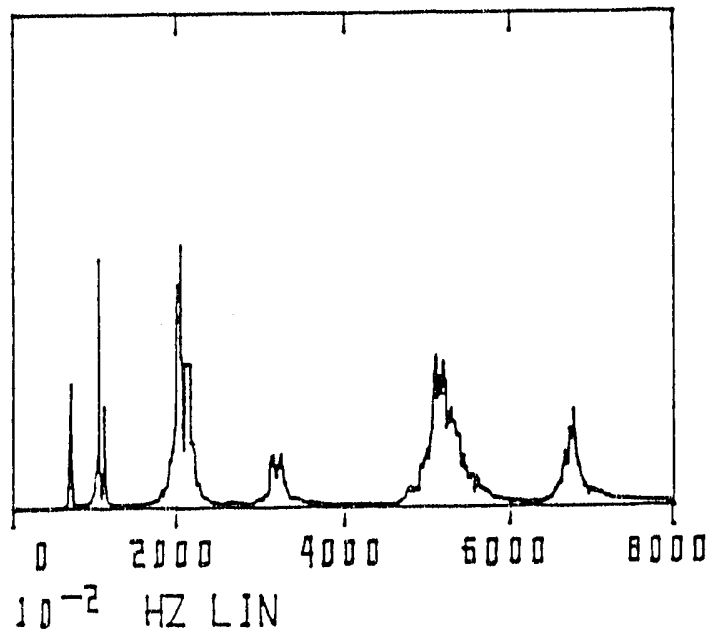

$X$ Magnet Response

Y Magnet Response

Z Magnet Response
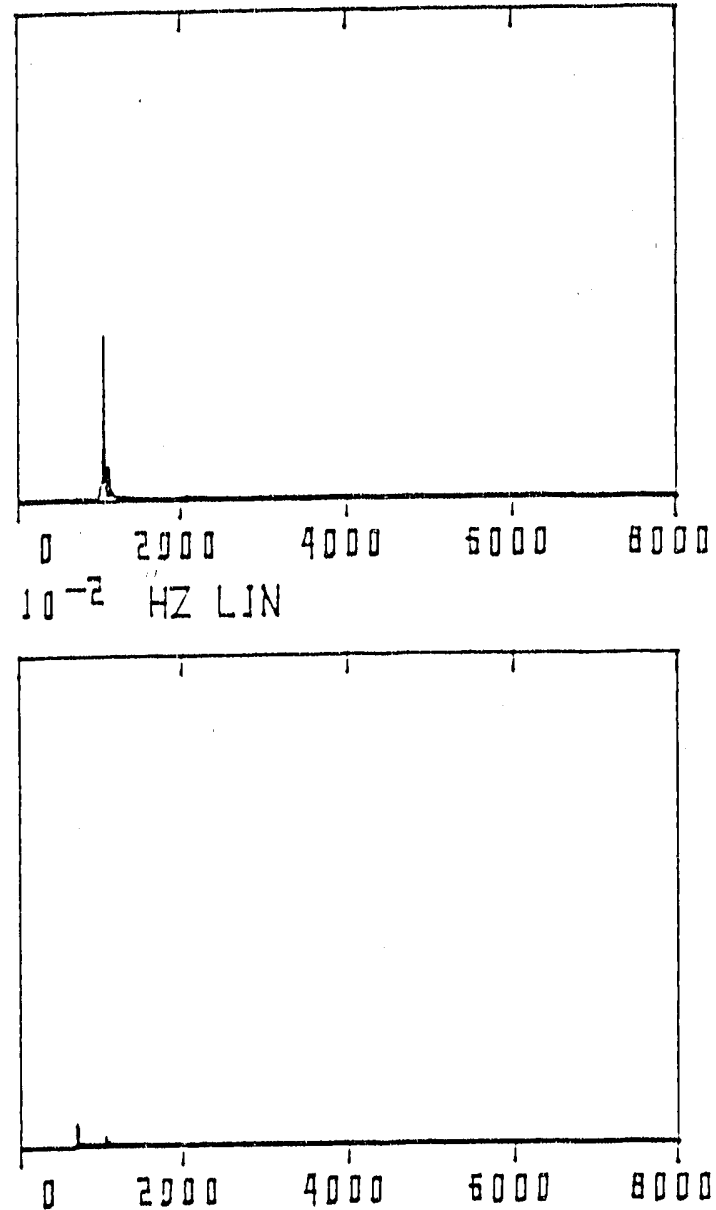
$10^{-2}$ HZ LIN

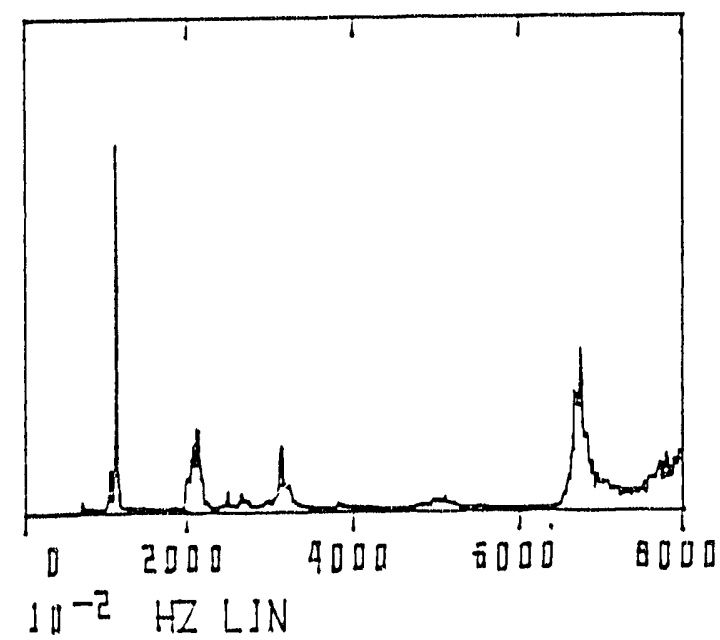
X Girder Response
Y Girder Response
Z Girder Response

TEST 1

$X$ Direction Excitation

Exutiation Levei $=0.6$ 


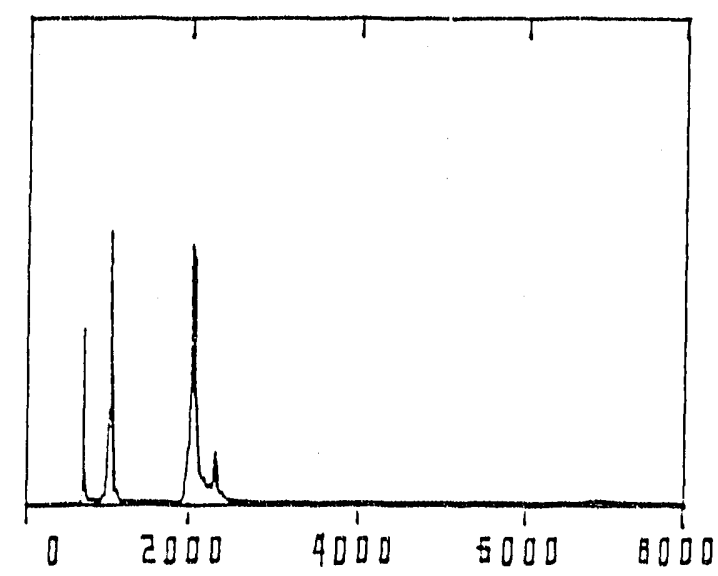

$10^{-2} H Z L I N$

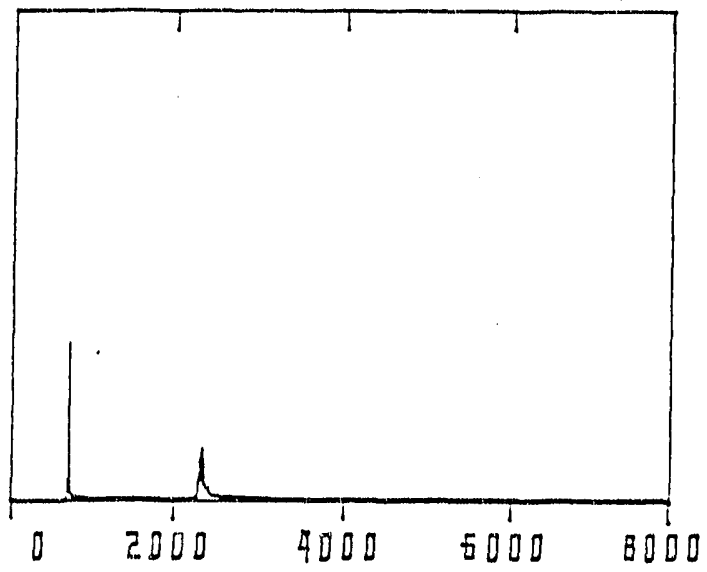

$10^{-2} \quad H Z$ LJN

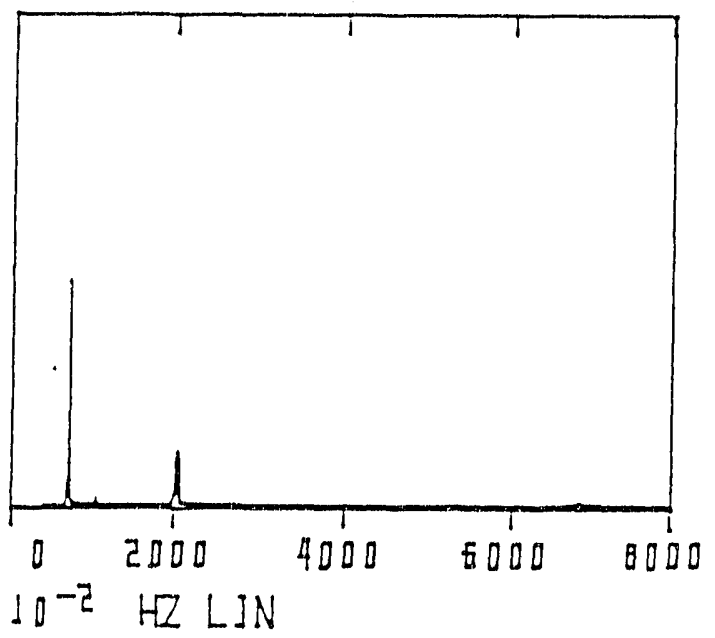

$x$ Magnet Response

X Girder Response

Y Magnet Response

Y Girder Response

2 Magnet Response

Z Girder Response

TEST 1

$Y$ Direction Excitation

Excitation Leve! = 0.6 


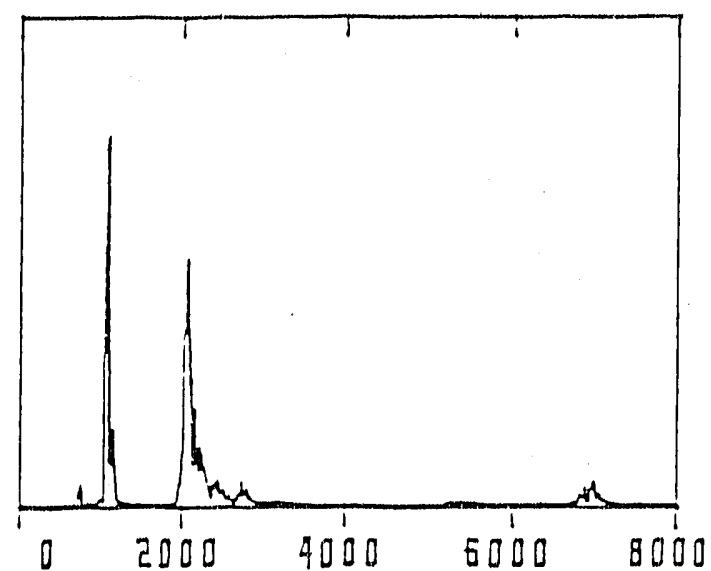

$10^{-2} \quad H Z$ LIN

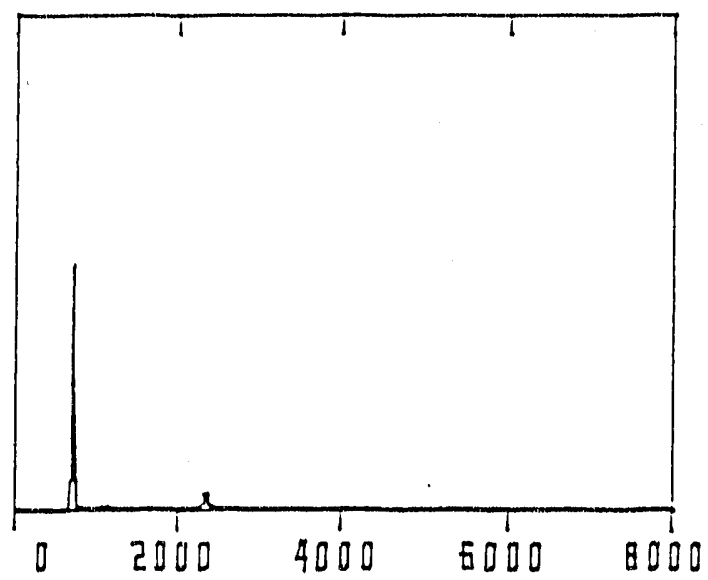

$10^{-2}$ HZ LIN

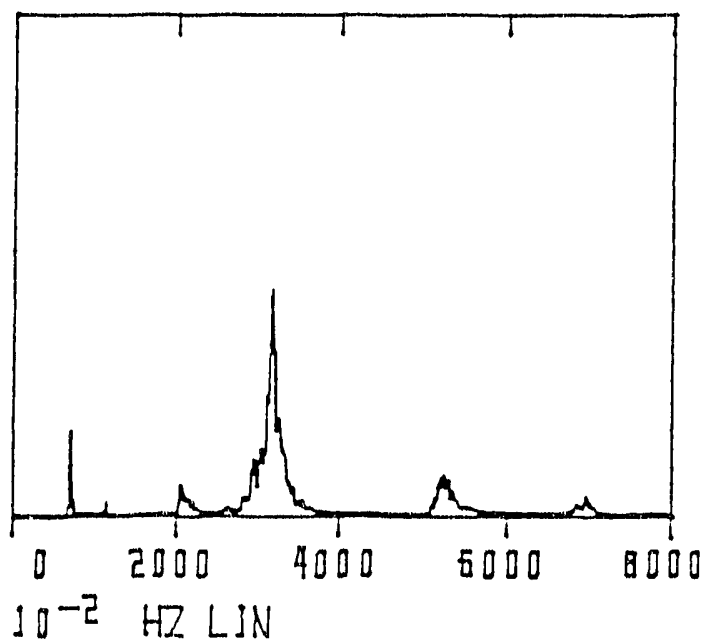

$\mathrm{X}$ Magnet Response

Y Magnet Response

Z Magnet Response

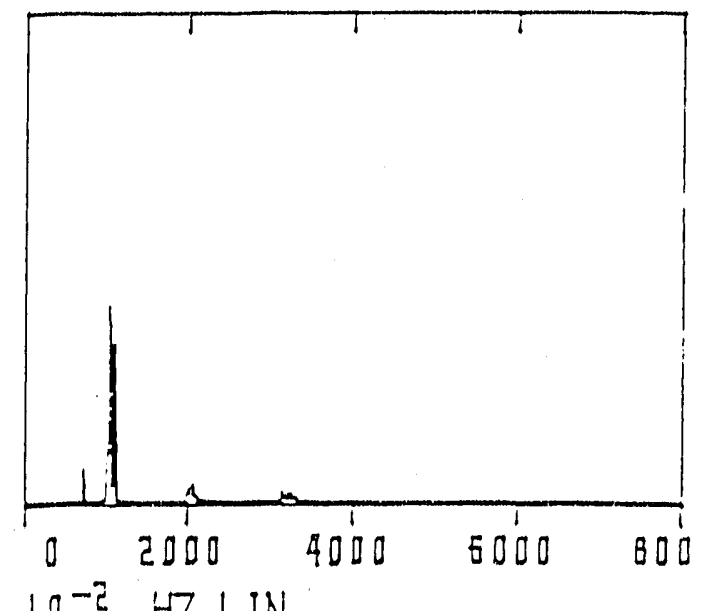

$10^{-2} H Z L I N$
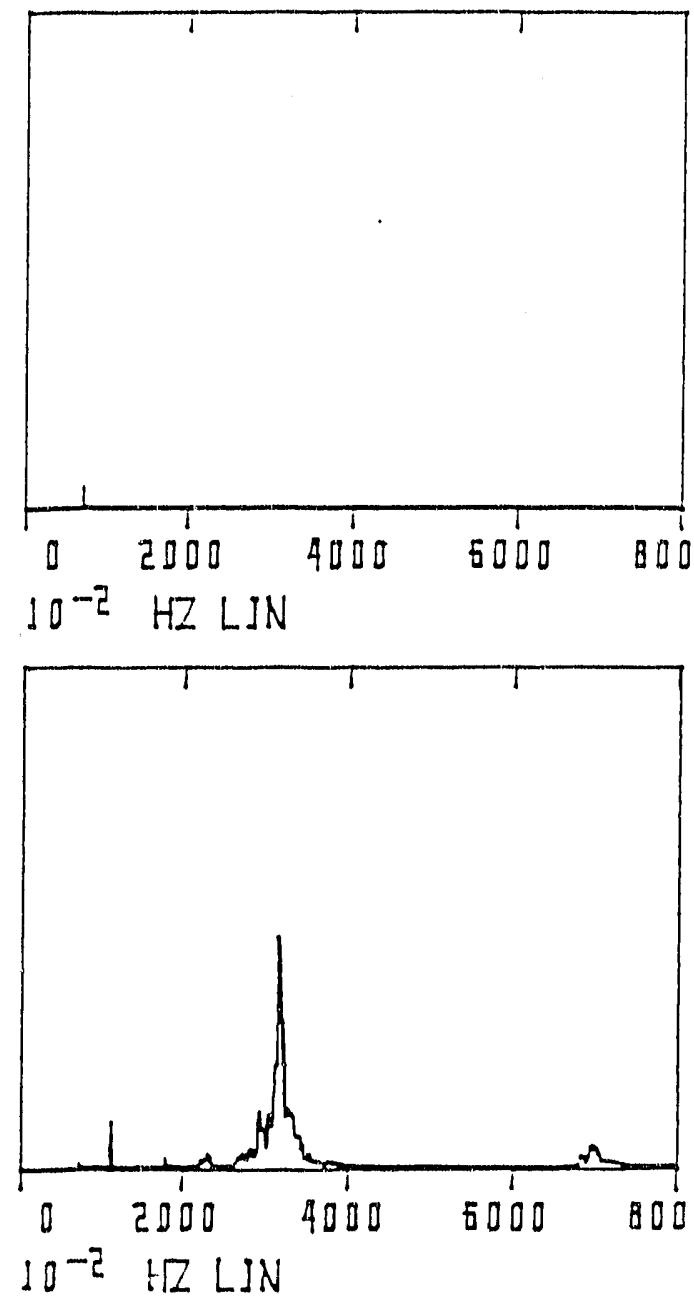

X Girder Response

Y Girder Response

Z Girder Response

Test 1

$Z$ Direction Excitation

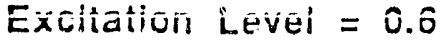



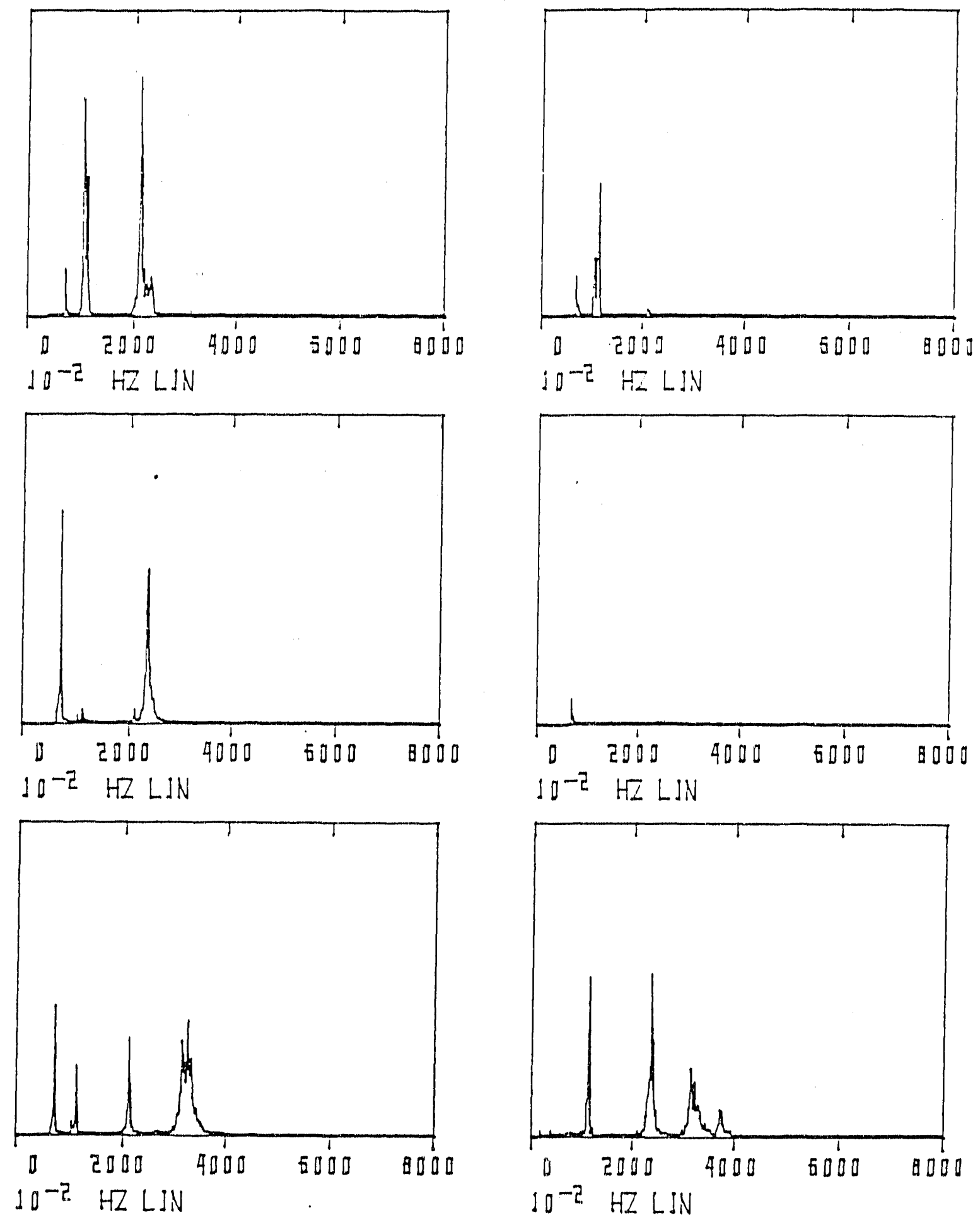

X Magnet Response

Y Magnet Response

$Z$ Magnet Response

$x$ Girder Response

Y Girder Response

2 Girder Response

TEST 2

$Y$ Direction Excitation

Excitation Level $=0.6$ 

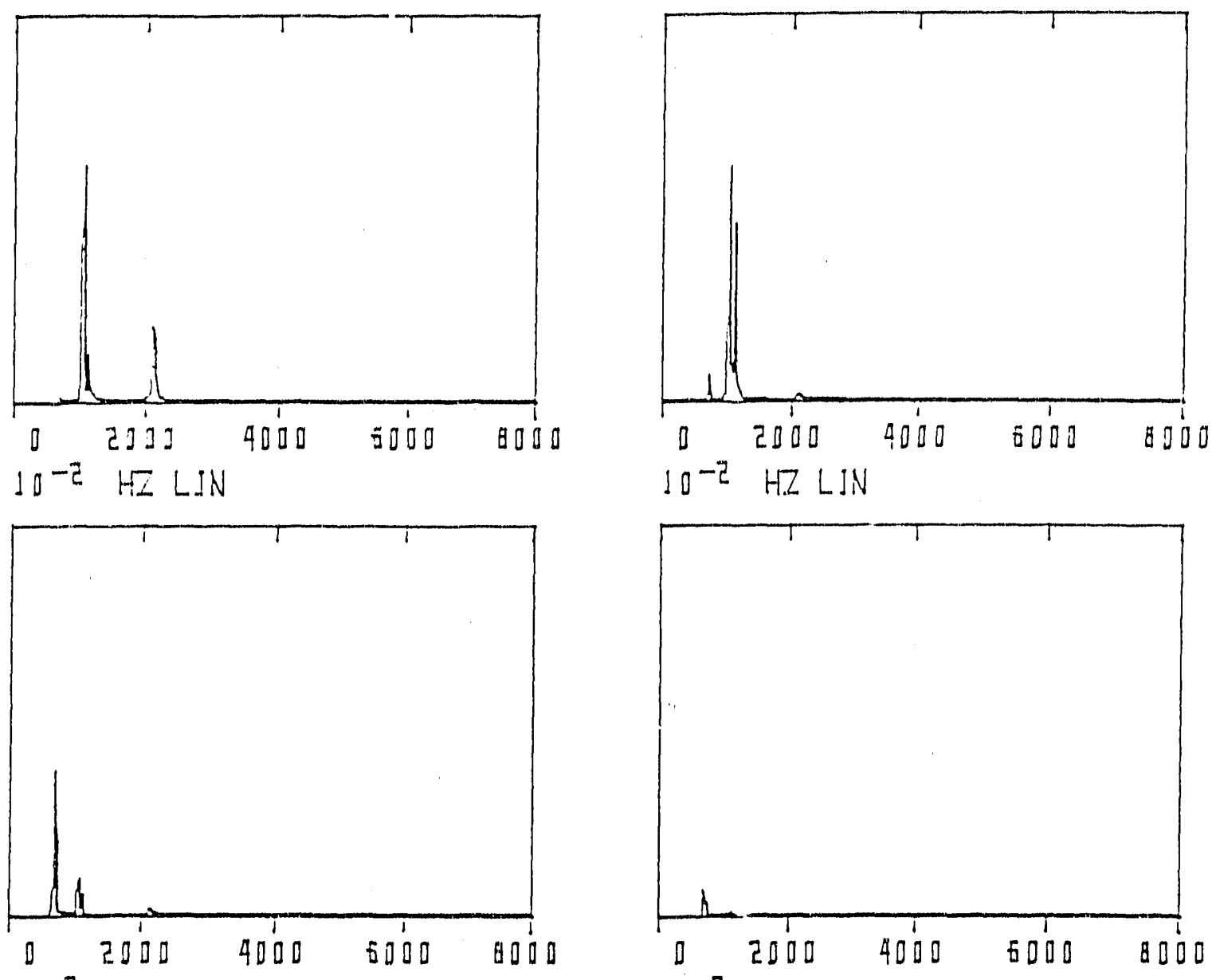

$10^{-2} H \angle L I N$
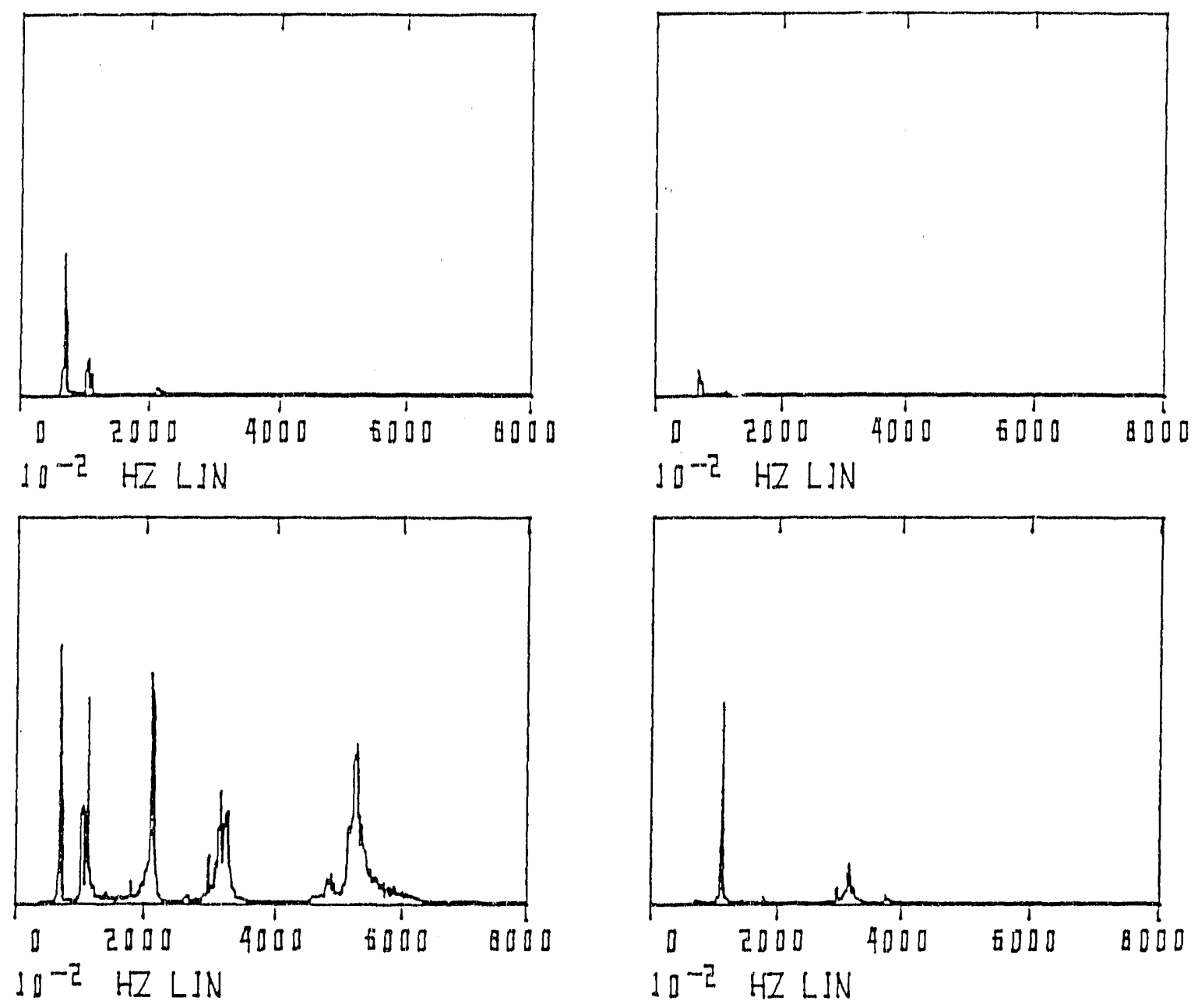

X Magnet Response

Y Magnet Response

Z. Magnet Response

X Girder Response

Y Girder Response

$Z$ Girder Response

TEST 2

$x$ Direction Exccitation

Excitation Level $=0.6$ 

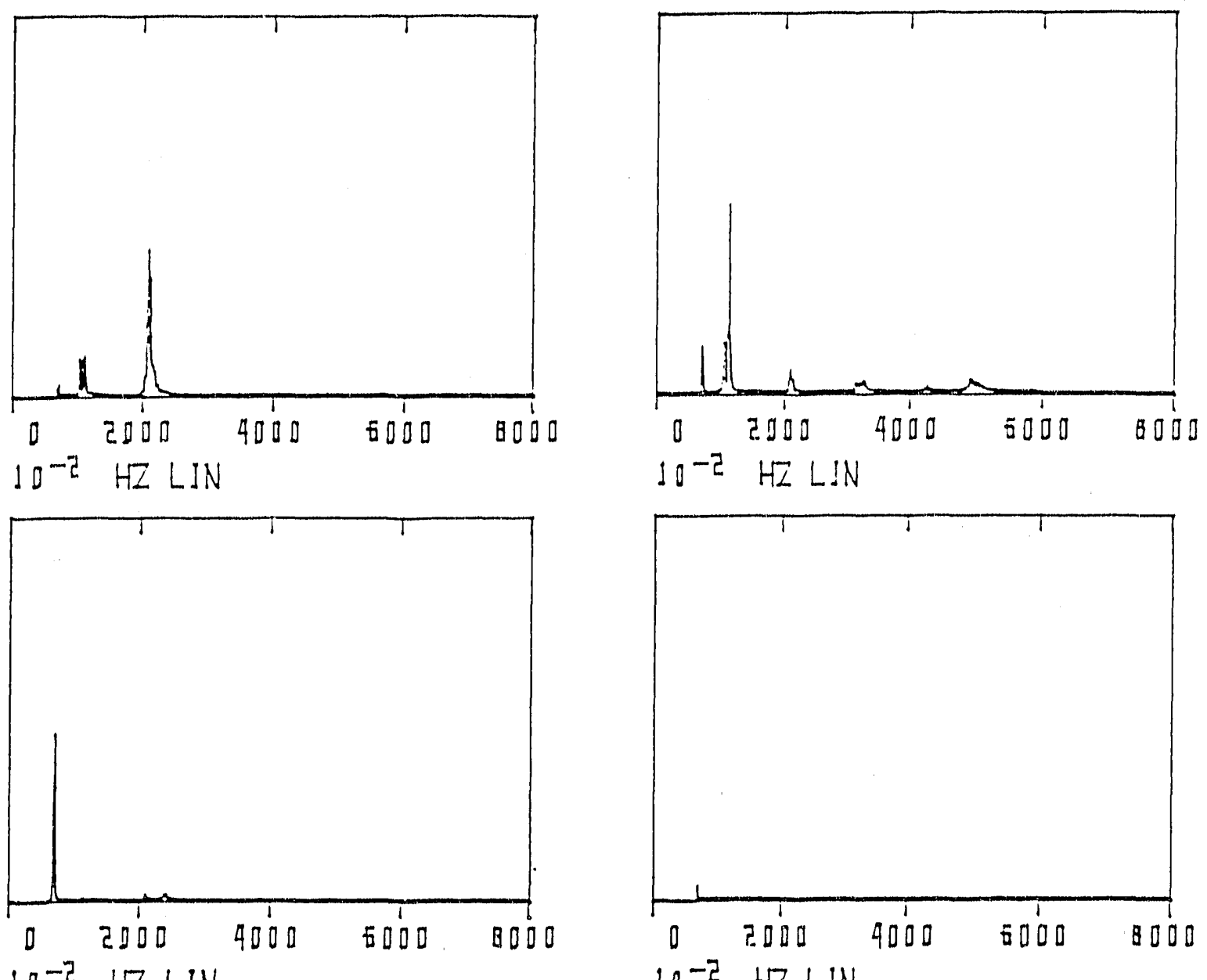
$10^{-2}$ HZ LIN
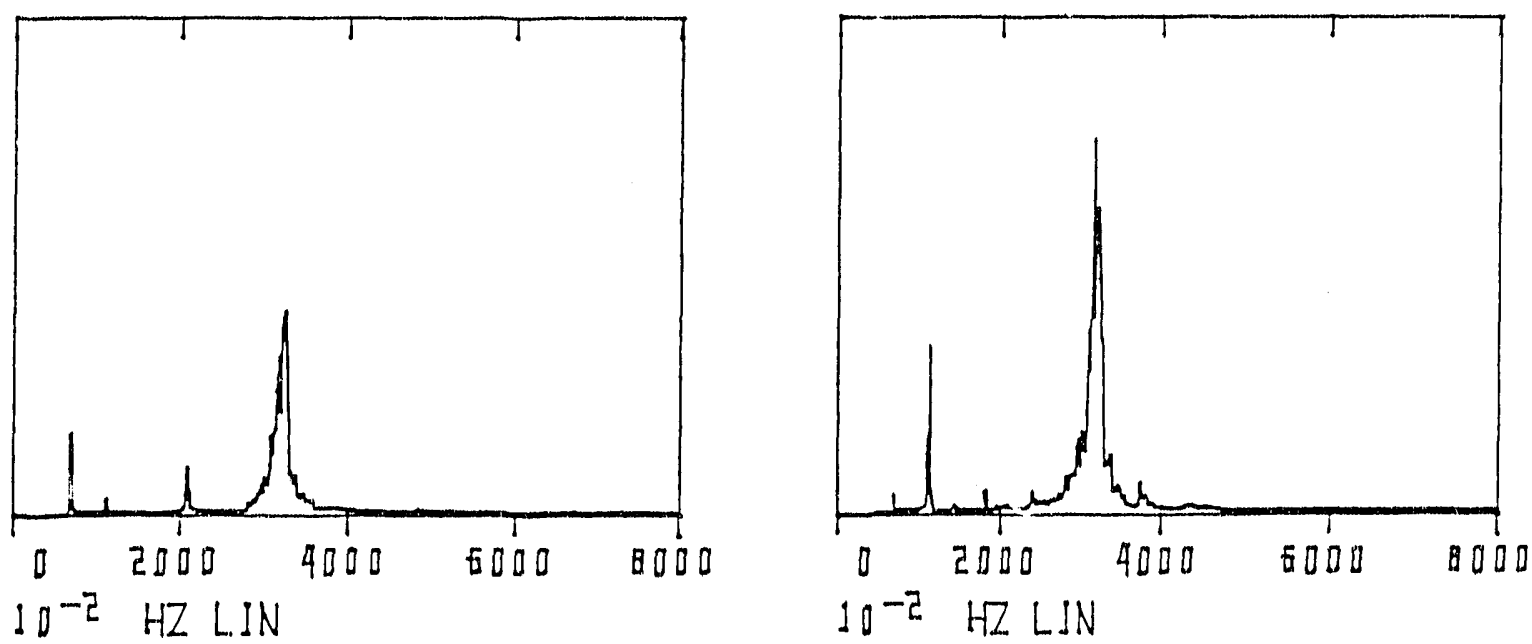

X Magnet Response

X Girder Response

Y Magnet Response

Y Girder Response

Z Magnet Response

2 Girder Response

TEST 2

$Z$ Direction Excitation

Excitation Level $=0.6$ 

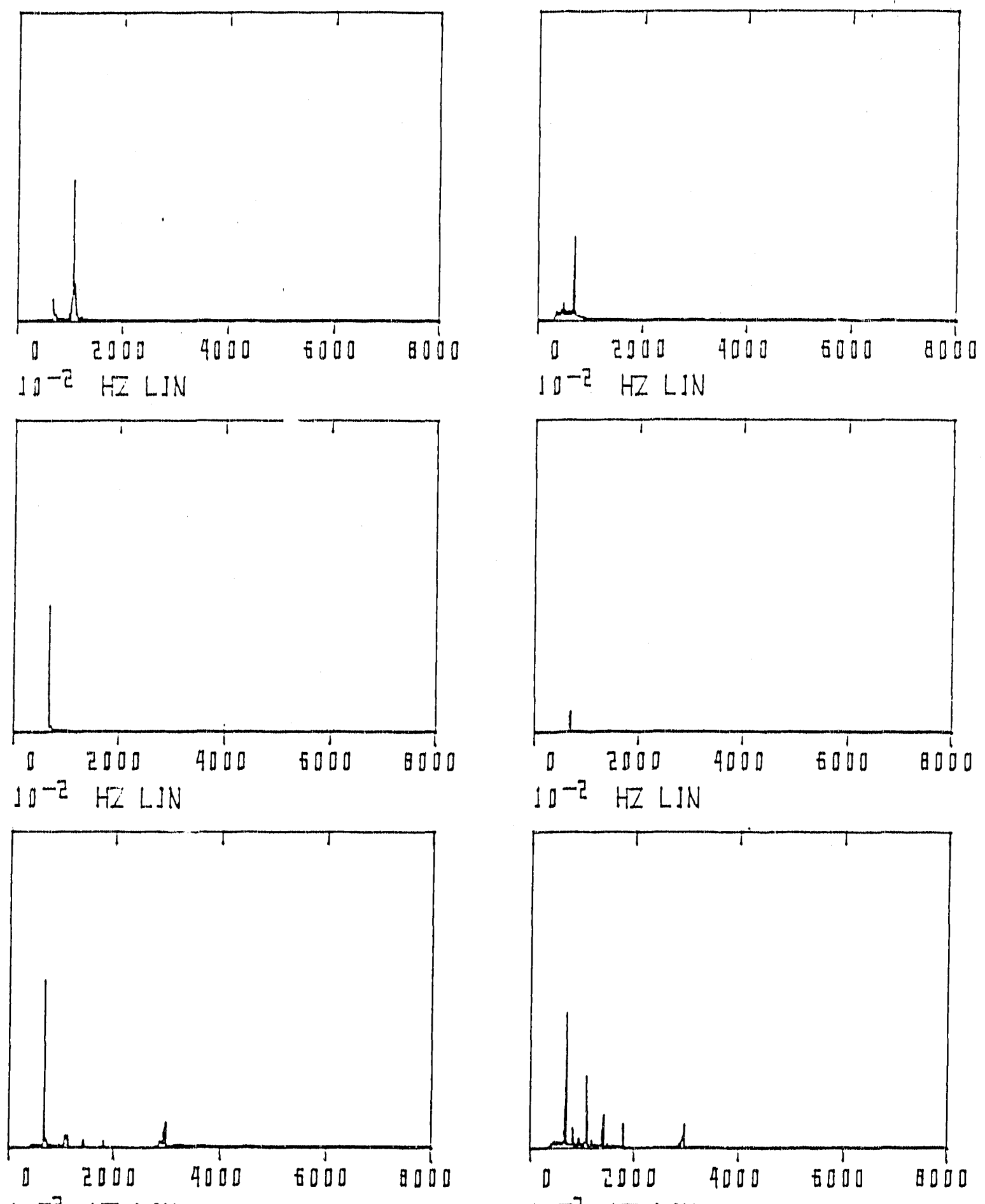

$10^{-2} \quad H Z L J N$

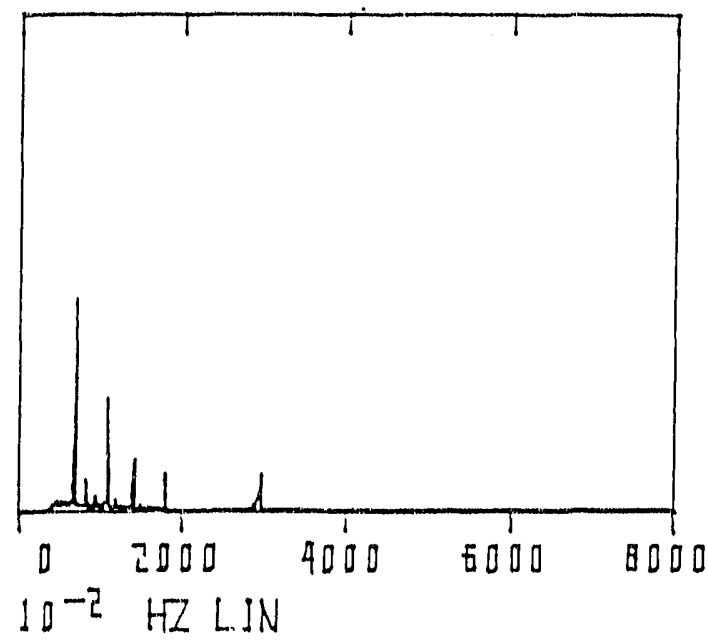

$X$ Magnet Response

$X$ Floor Response

Y Magnet Response

Y Floor Response

Z Magnet Response

Z Floor Response

TEST 3

Ambient Response

Data: 4/4/91, 3:35 PM 

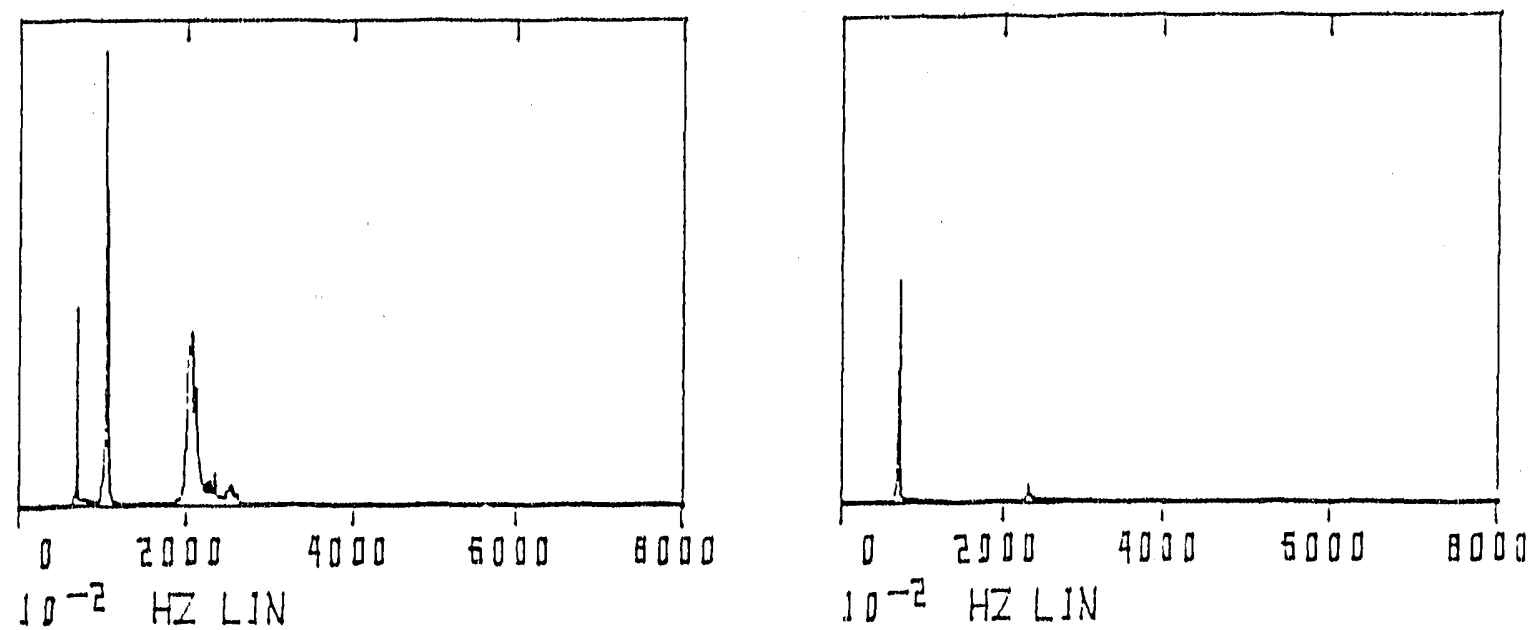

$10^{-2}$ HZ LIN

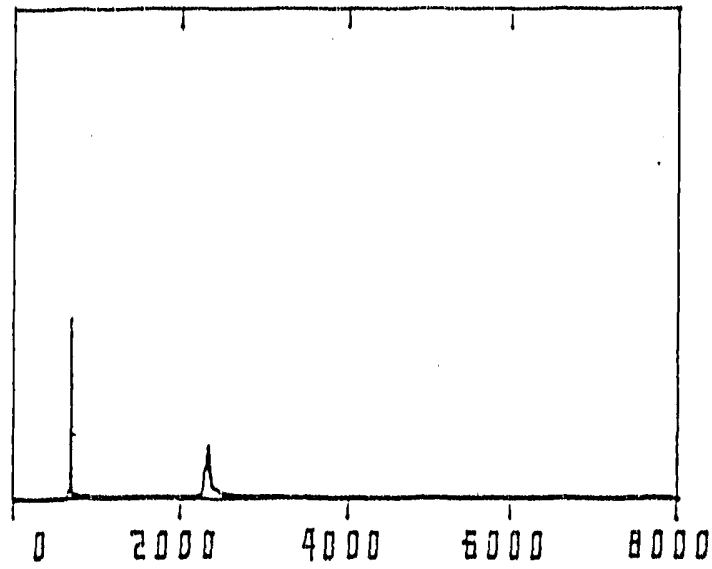

$10^{-2} \quad H Z L I N$

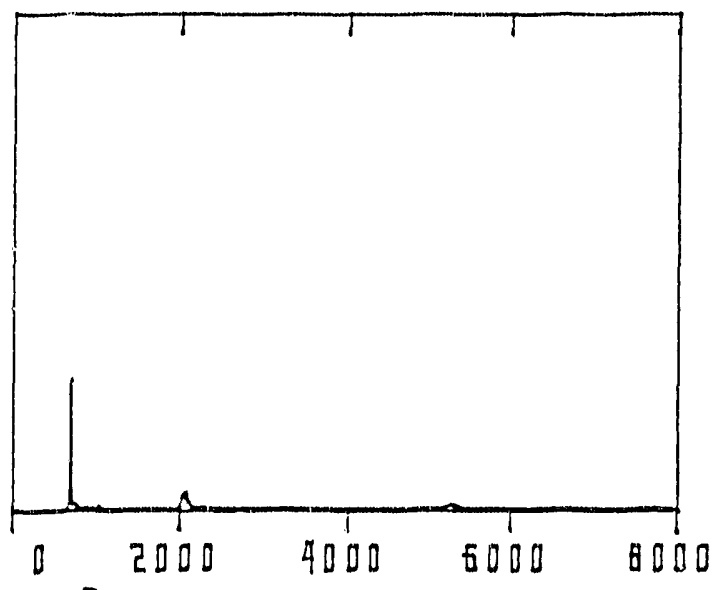

$10^{-2} H Z L I N$

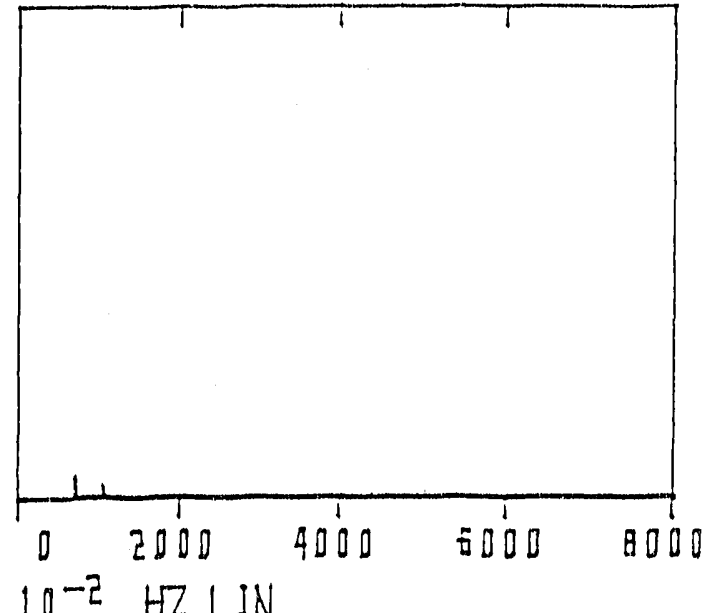

$10^{-2} H Z \operatorname{LIN}$

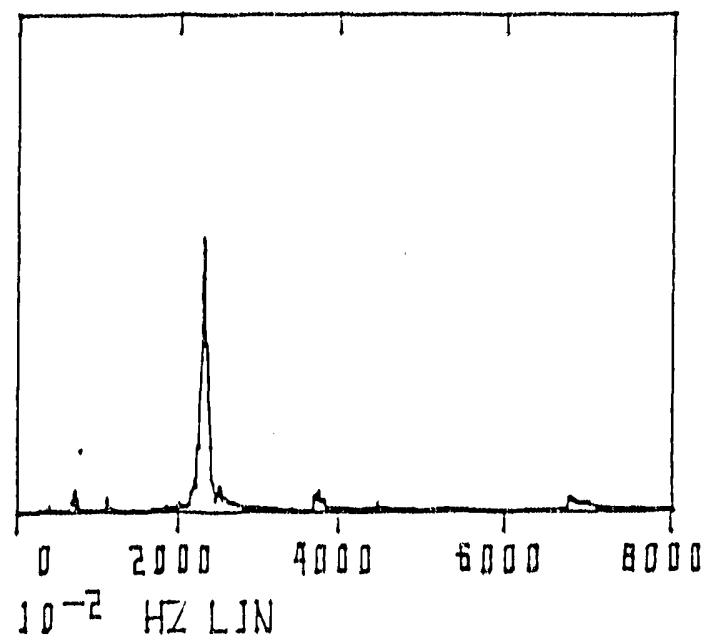

X Magnet Response

$x$ Girder Response

Y Magnet Response

Y Girder Response

$Z$ Magnet Response

Z Girder Desponse

TEST 5

$Y$ Direction Excitation

Excitation Level $=0.6$ 


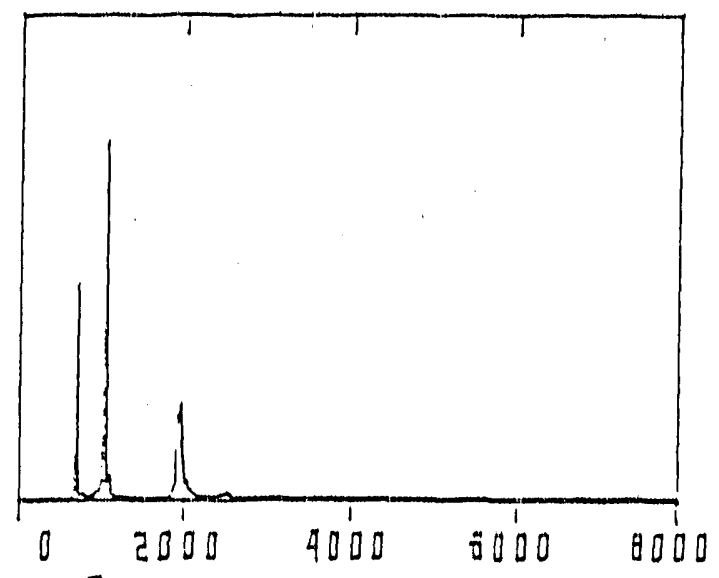

$10^{-E} H Z$ LIN
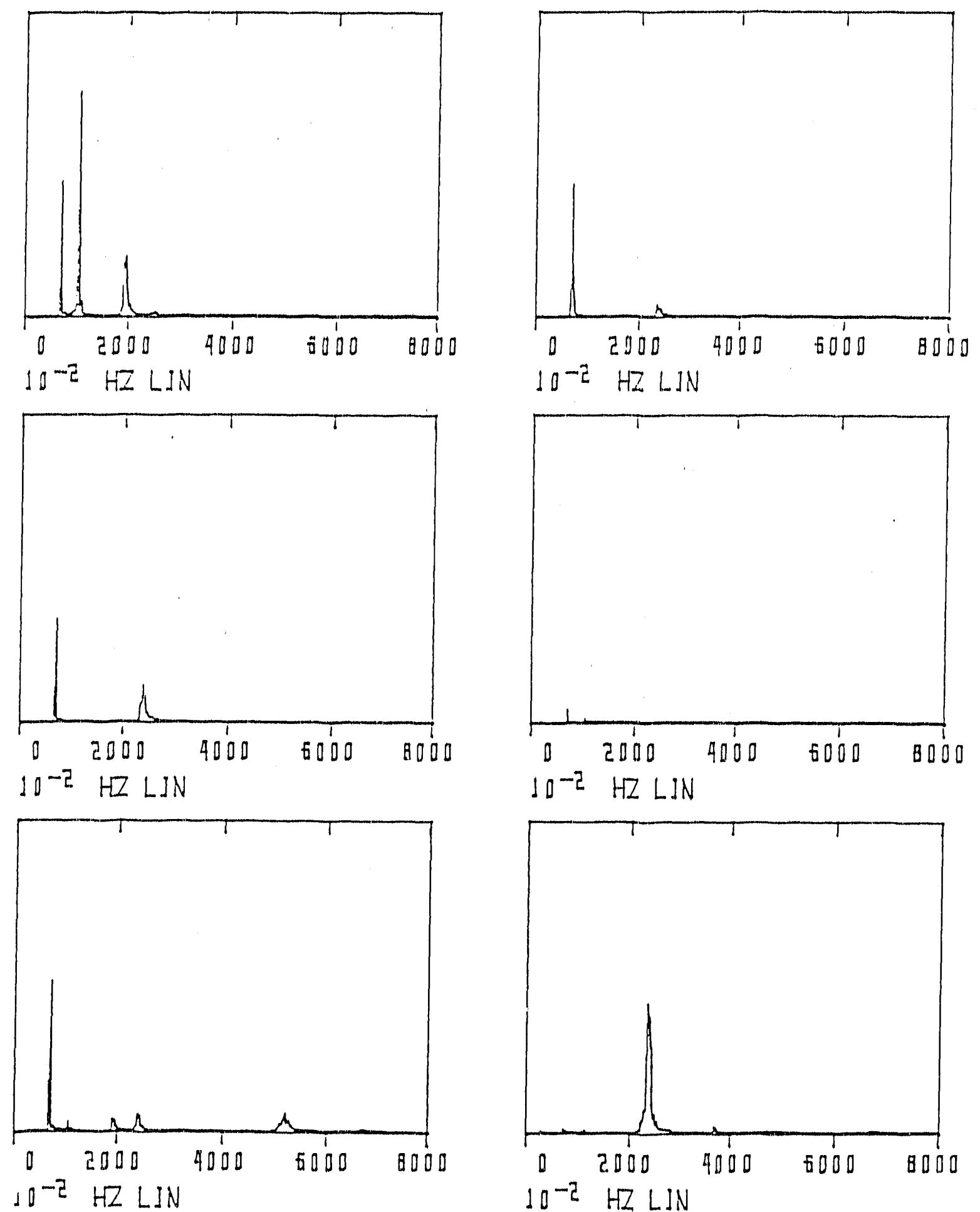

X Magnet Response

Y Magnet Response

Z Magnet Respons:
X Girder Response

Y Girder Response

2 Girder Response 
Appendix C

RMS Displacements and Transfer Functions for Phase I 'Tests 
RMS,Test1,Wldeband

\begin{tabular}{|c|c|c|c|c|c|c|c|}
\hline ExDIr & ExLeV & $\# 1,(X, M)$ & $\# 2,(Y, M)$ & $\# 3,(Z, M)$ & $\# 4,(x, Q)$ & $\# 5,(Y, G)$ & $\# G,(Z, G)$ \\
\hline & & Imic. RMS & mlc. PMS & mlc. RMS & mic. RMS & Imlc. RMS & mic. RMS \\
\hline$x$ & 0.6 & 1.4611 & 1.4166 & 0.5855 & 0.6887 & 0.6366 & $\quad 0.2520$ \\
\hline$y$ & 0.4 & $1.0505 i$ & 0.9796 & 0.3602 & 0.4458 & $0.4772 !$ & 0.1555 \\
\hline$x$ & 0.1 & $0.2372 !$ & 0.6339 & 0.11881 & 0.1162 & 0.3527 & 0.0585 \\
\hline$y$ & 0.6 & 1.0256 & 3.2986 & 0.4301 & 0.4480 & 1.3510 & 0.3384 \\
\hline$y$ & 0.4 & 0.69291 & 2.1281 & 0.2922 & 0.31681 & 0.8881 & 0.2436 \\
\hline$y$ & 0.1 & 0.2267 & 0.7984 & 0.12261 & 0.1209 & 0.39641 & 0.0721 \\
\hline$z$ & 0.6 & 0.40361 & 0.7599 & 0.4058 & 0.1597 & 0.37101 & 0.1863 \\
\hline$z$ & 0.4 & 0.2677 & 0.6924 & 0.27881 & 0.0984 & 0.35501 & 0.1295 \\
\hline$\underline{z}$ & 0.1 & 0.1391 & 0.6034 & 0.11101 & 0.082 .61 & 0.3354 & 0.0521 \\
\hline & & & & & & & \\
\hline & & & & & & & \\
\hline & & & & & & & \\
\hline & & & & & & & \\
\hline & & & & & & & \\
\hline & & & & & & & \\
\hline & & & & & & & \\
\hline & & & & & & & \\
\hline & & & & & & & \\
\hline & & & & & & & \\
\hline & & & & & & & \\
\hline & & & & & & & \\
\hline & & & & 1 & & & \\
\hline
\end{tabular}


RMS, Test2, Wideband

\begin{tabular}{|c|c|c|c|c|c|c|c|}
\hline EXD/r & ExLeV & $\$ 1,(X, M)$ & $\# 2,(Y, M)$ & $\# 3,(Z, M)$ & $\# 4,(X, G)$ & $\# 5,(Y, G)$ & $(\bar{z}, \mathbf{G})$ \\
\hline & & mic. RMS & mic. RMS & m/c. FMS & mic. RMS & mic. RMS & mic. RMS \\
\hline$z$ & 0.61 & 0.3598 & 0.7175 & 0.3495 & 0.1773 & 0.3668 & 0.1547 \\
\hline$z$ & 0.4 & 0.2622 & 0.6203 & 0.2357 & 0.1263 & 0.32431 & 0.1065 \\
\hline$z$ & 0.1 & 0.1978 & 0.5723 & 0.11671 & 0.1158 & 0.3202 & 0.0653 \\
\hline$x$ & 0.6 & 0.78261 & 0.8717 & 0.2938 & 0.3921 & 0.4627 & 0.1109 \\
\hline$x$ & 0.4 & 0.5559 & 0.7537 & 0.1950 & 0.2897 & 0.41121 & 0.0790 \\
\hline$x$ & 0.1 & 0.19111 & 0.5822 & 0.0978 & 0.10901 & 0.32891 & 0.0451 \\
\hline$y$ & 0.61 & 1.19621 & 2.8558 & 0.5795 & 1.8688 & 1.06611 & 0.3854 \\
\hline$y$ & 0.4 & 0.71201 & 1.7436 & 0.3683 & 0.3514 & 0.7116 & 0.2279 \\
\hline$y$ & 0.1 & 0.19501 & 0.6659 & 0.1555 & 0.1069 & $0.3452 !$ & 0.0743 \\
\hline & & & & & & & \\
\hline & & & & & & & \\
\hline & & & & & & & \\
\hline & & & & & & & \\
\hline & & & & & & & \\
\hline & & & & & & & \\
\hline & & & & & & & \\
\hline & & & & & & & \\
\hline & & & & & & & \\
\hline & & 1 & & & & & \\
\hline & & & & & & & \\
\hline & & & & & & & \\
\hline & & & & & & & \\
\hline
\end{tabular}


Test 3, AMBIENT, Wideband

\begin{tabular}{|c|l|r|r|r|}
\hline & \multicolumn{1}{|c|}{ A } & \multicolumn{1}{|c|}{ B } & \multicolumn{1}{c|}{ C } & \multicolumn{1}{c|}{ D } \\
\hline 1 & Location & Amp Galn & MS Volts & RMS microns \\
\hline 2 & $x \mathrm{~m}$ & 10 & 0.001251 & 0.11360676 \\
\hline 3 & $y \mathrm{~m}$ & 10 & 0.02686 & 0.52641535 \\
\hline 4 & $2 \mathrm{~m}$ & 10 & 0.0007353 & 0.08709793 \\
\hline 5 & $x f$ & 100 & 0.04901 & 0.07110791 \\
\hline 6 & $y f$ & 100 & 0.02712 & 0.0528957 \\
\hline 7 & $z f$ & 100 & 0.00987 & 0.03191054 \\
\hline 8 & & & & \\
\hline 9 & & & & \\
\hline 10 & TFx & 1.59766714 & & \\
\hline 11 & TFy & 9.95194945 & & \\
\hline 12 & TFz & 2.72944097 & & \\
\hline
\end{tabular}




\begin{tabular}{|c|c|c|c|c|c|c|c|}
\hline EXDir & ExLev & $\# 1,(X, M)$ & $\# 2,(Y, M)$ & $\# 3,(Z, M)$ & $\# 4,(X, G)$ & $\# 5,(Y, G)$ & \# $G,(Z, G)$ \\
\hline & & mic. RMS & mic. RMS & Imic. RMS & mic. RMS & mic. RMS & Imic. RMS \\
\hline$y$ & 0.6 & 1.1364 & 5.2658 & 0.5002 & 7.1130 & 5.2912 & 0.3571 \\
\hline & & & & & I & & \\
\hline & & & & & 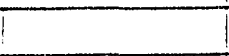 & & \\
\hline & & & & & I & & \\
\hline & & & & & $\vdots$ & & \\
\hline & & & & & I & & \\
\hline & & & & & 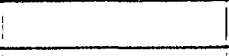 & & \\
\hline & & & & & 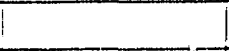 & & \\
\hline & & & & & I & & \\
\hline & & & & & $!$ & 1. & \\
\hline & & & & & I & & \\
\hline & & & & & I & & \\
\hline & & & & & I & & \\
\hline & & & & & I & & \\
\hline & & & & & 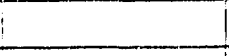 & & \\
\hline & & & & & 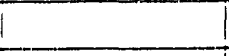 & & \\
\hline & & & & & $i$ & & \\
\hline & & & & & I & & \\
\hline & & & & & 1 & & \\
\hline & & & & & & & \\
\hline & & & & & 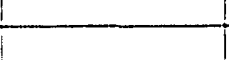 & & \\
\hline
\end{tabular}


RMS, Test6, Wideband

\begin{tabular}{|c|c|c|c|c|c|c|c|}
\hline EXDir & ExLeV & $\# y,(X, M)$ & $\# 2,(Y, M)$ & $\# 3,(Z, M)$ & $\# 4,(X, G)$ & $\# 5,(Y, G)$ & $\# 6,(Z, G)$ \\
\hline & & mic. RMS & imic. RMS & mic. RMS & mic. RMS & mic. RMS & mic. RMS \\
\hline y & 0.6 & 0.8935 & 3.8382 & 0.5176 & 1.4639 & 0.5173 & 0.4331 \\
\hline$y$ & 0.4 & 0.6408 & 2.8231 & 0.3800 & 1.0896 & 0.3784 & 0.2974 \\
\hline$y$ & 0.1 & 0.2685 & 0.9059 & 0.1944 & 0.4177 & 0.1850 & 0.1455 \\
\hline & & 1 & 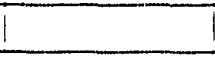 & $I$ & & & \\
\hline & & 1 & 1 & $i$ & & & \\
\hline & & 1 & 1 & 1 & & & \\
\hline & 1 & 1 & I & 1 & & & \\
\hline & & & & & & & \\
\hline & & I & I & & & & \\
\hline & & & & & & & \\
\hline & & & & & & & \\
\hline & & & & & & & \\
\hline & & & & & & & \\
\hline & & 1 & & & & & \\
\hline & & & & & & & \\
\hline & & & & & & & \\
\hline & & T & i & & & & \\
\hline & & $T$ & r & & & & \\
\hline & & & & & & & \\
\hline & & & & & & & \\
\hline & & & & & & & \\
\hline & $A \vee G$ & \#DIV/OI & $\# 01 \bar{V} / 0 !$ & \#DIV/O! & & & \\
\hline
\end{tabular}


RMS, TF, Test1, $7.62 \mathrm{~Hz}$

\begin{tabular}{|c|c|c|c|c|c|c|c|}
\hline EXDir & ExLev & $\# 1,(X, M)$ & $\# 2,(Y, M)$ & $\# 3,(Z, M)$ & $\# 4,(X, G)$ & $\# 5,(Y, G)$ & $\# 6,(Z, G)$ \\
\hline & & mic. RMS & mic. RMS & mic. RMS & mic. RMS & mic. RMS & mic. RMS \\
\hline$x$ & 0.6 & 0.1090 & 0.8000 & 0.0973 & 0.0820 & 0.4362 & 0.0152 \\
\hline $\bar{x}$ & $0 . \overline{4}$ & 0.0729 & 0.6788 & $0.0786 i$ & 0.0560 & 0.3787 & 0.0125 \\
\hline$x$ & 0.1 & 0.0406 & 0.5973 & 0.06701 & 0.0322 & 0.3354 & 0.0108 \\
\hline$y$ & 0.6 & 0.3108 & 2.0956 & 0.2553 & 0.2816 & 1.1126 & 0.0381 \\
\hline$y$ & 0.4 & 0.1897 & 1.3894 & 0.1605 & 0.1731 & 0.7391 & 0.0255 \\
\hline$y$ & 0.1 & 0.0577 & 0.6535 & 0.08091 & 0.0485 & 0.3641 & 0.0122 \\
\hline$z$ & 0.6 & 0.0459 & 0.5907 & 0.0691 & 0.0383 & 0.33191 & 0.0109 \\
\hline$z$ & 0.4 & 0.0387 & 0.5998 & 0.0647 & 0.0336 & 0.3336 & 0.0104 \\
\hline$z$ & 0.1 & 0.0395 & $0 . \overline{5702}$ & 0.0655 & 0.0335 & 0.32001 & 0.0102 \\
\hline & & & & & 2 & 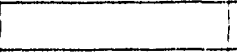 & \\
\hline & & & & & & & \\
\hline & & & & & 7 & 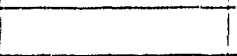 & \\
\hline & & & & & 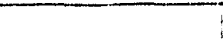 & 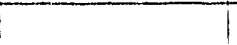 & \\
\hline & & & & & T & 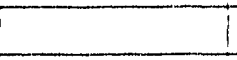 & \\
\hline & & & & & & 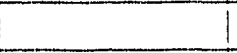 & \\
\hline & & & & & 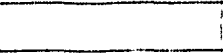 & 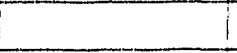 & \\
\hline & & & & & 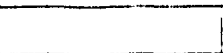 & $T$ & \\
\hline & & & & & 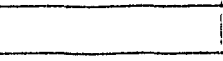 & 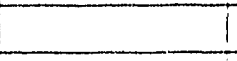 & \\
\hline & & & & & & 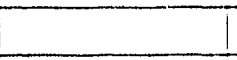 & \\
\hline & & & & & & & \\
\hline & & & & & & & \\
\hline & & & & & & & \\
\hline
\end{tabular}


RMS, TF,Test2,7.62. Hz

\begin{tabular}{|c|c|c|c|c|c|c|c|}
\hline EXDir & ExLeV & $\# 1,(X, M)$ & $\# 2,(Y, M)$ & $\# 3,(Z, M)$ & $\# 4,(X, G)$ & $\# 5,(Y, G)$ & $\# 6,(Z, G)$ \\
\hline & & mic. RMS & mic. RMS & mic. RMS & mic. FMS & mic. RMS & mic. RMS \\
\hline$z$ & 0.6 & 0.0564 & 0.5859 & $0.0716 i$ & 0.0491 & 0.3288 & 0.0117 \\
\hline$z$ & 0.4 & 0.0378 & 0.5336 & 0.0651 & 0.02901 & 0.3007 & 0.0105 \\
\hline$z$ & 0.1 & 0.0441 & 0.5211 & 0.0625 & 0.0348 & 0.2969 & 0.0111 \\
\hline$x$ & $0.6 i$ & 0.08601 & 0.67501 & 0.08751 & 0.0751 & 0.3743 & 0.0119 \\
\hline$x$ & 0.4 & 0.04581 & 0.6507 & 0.06861 & $0.0442 !$ & 0.3644 & 0.0101 \\
\hline$x$ & 0.1 & 0.03901 & 0.5545 & 0.06431 & 0.0321 & 0.3145 & 0.0110 \\
\hline$y$ & 0.6 & $0.2142 !$ & 1.4741 & 0.18301 & 0.19311 & 0.7858 & 0.0281 \\
\hline$y$ & 0.4 & $0.1416 i$ & 1.0287 & 0.1217 & 0.12581 & 0.5610 & 0.0149 \\
\hline \multirow[t]{3}{*}{ y } & 0.1 & 0.0617 & 0.55531 & 0.06581 & 0.05151 & 0.3115 & 0.0099 \\
\hline & & & & & & & \\
\hline & & $T F, x$ & $T F, y$ & $T F, z$ & & & \\
\hline$z$ & 0.6 & 1.15 & 1.781 & 6.14 & & & \\
\hline$z$ & 0.4 & 1.30 & 1.77 & $6.21 i$ & & & \\
\hline$z$ & 0.1 & $1.27 !$ & 1.751 & 5.61 & & & \\
\hline$\underline{x}$ & 0.6 & $1.15 i$ & 1.801 & $7.34 i$ & & & \\
\hline$\underline{x}$ & 0.4 & 1.04 & 1.79 & 6.79 & & & \\
\hline$x$ & 0.1 & 1.211 & 1.76 & 5.831 & & & \\
\hline y & 0.6 & 1.11 & 1.88 & 6.51 & & & \\
\hline$y$ & 0.4 & 1.13 & 1.831 & 8.191 & & & \\
\hline y & 0.1 & 1.20 & 1.78 & 6.631 & & & \\
\hline & & & & & & & \\
\hline & $A \vee G$ & 1.17 & 1.80 & 6.58 & & & \\
\hline
\end{tabular}


Test 3, AMBIENT,7.62 Hz

\begin{tabular}{|c|l|r|r|r|}
\hline & \multicolumn{1}{|c|}{ A } & \multicolumn{1}{c|}{ B } & \multicolumn{1}{c|}{ C } & \multicolumn{1}{c|}{ D } \\
\hline 1 & Location & Amp Gain & MS Volts & RMS microns \\
\hline 2 & $x \mathrm{~m}$ & 10 & 0.0001313 & 0.03680509 \\
\hline 3 & $y \mathrm{~m}$ & 10 & 0.02422 & 0.49987637 \\
\hline 4 & $\mathrm{zm}$ & 10 & 0.00007722 & 0.02822542 \\
\hline 5 & $x f$ & 100 & 0.01777 & 0.0428173 \\
\hline 6 & $y f$ & 100 & 0.01496 & 0.03928632 \\
\hline 7 & $z f$ & 100 & $1.776 E-06$ & 0.00042805 \\
\hline 8 & & & & \\
\hline 9 & & & & \\
\hline 10 & TFx & & & \\
\hline 11 & TFy & 0.85958465 & & \\
\hline 12 & TFz & 12.72393 & & \\
\hline
\end{tabular}


RIMS, TF, Test5,7.62 Hz

\begin{tabular}{|c|c|c|c|c|c|c|c|}
\hline EXDir & ExLev & $\# 1,(X, M)$ & $\# 2,(Y, M)$ & $\# 3,(Z, M)$ & $\# 4,(X, G)$ & $\# 5,(Y, G)$ & $\# 6,(Z, G)$ \\
\hline & & mic. RMS & mic. RMS & mic. RMS & mic. RMS & mic. RMS & mic. RMS \\
\hline$y$ & 0.6 & $0.3322 !$ & $\quad 2.2226$ & 0.5863 & 1.1144 & 0.3414 & 1.6279 \\
\hline & & & & & & & \\
\hline & & T & & & & & \\
\hline & & $T$ & & & & 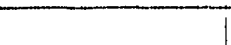 & 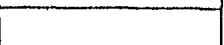 \\
\hline & & 1 & & & & 1 & \\
\hline & & I & & & & 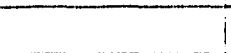 & \\
\hline & & 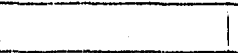 & & & & & \\
\hline & & & & & & & \\
\hline & & & & & & $\bar{i}$ & \\
\hline & & & & & & & \\
\hline & & $T F, x$ & TF,y & $T F, z$ & & & \\
\hline$y$ & 0.6 & 0.301 & 6.51 & 0.36 & & & \\
\hline & & 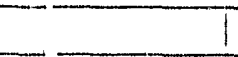 & & 1 & & & \\
\hline & & 1 & & & & & \\
\hline & & 1 & & & & i & \\
\hline & & & & & & & \\
\hline & & & & & & & \\
\hline & & & & & & & \\
\hline & & & & & & & \\
\hline & & & & & & & \\
\hline & & & & & & & \\
\hline & & & & & & & \\
\hline
\end{tabular}


RMS, TF, Test6, $7.62 \mathrm{~Hz}$

\begin{tabular}{|c|c|c|c|c|c|c|c|}
\hline EXDir & ExLEV & $\# 1,(X, M)$ & $\# 2,(Y, M)$ & $\# 3,(Z, M)$ & $\# 4,(X, G)$ & $\# 5,(Y, G)$ & $\# 6,(Z, G)$ \\
\hline & & mic. RMS & mic. RMS & mic. RMS & mic. RMS & mic. RMS & mic. AMS \\
\hline$y$ & 0.6 & 0.3555 & 2.2923 & 0.2768 & 1.1669 & 0.3435 & 0.0387 \\
\hline$y$ & 0.4 & 0.2555 & 1.7282 & 0.1902 & 0.88631 & 0.2442 & 0.0282 \\
\hline$y$ & 0.1 & 0.0646 & 0.65961 & 0.0801 & 0.3493 & 0.0579 & 0.0167 \\
\hline & & & & & & & \\
\hline & & & & & & & \\
\hline & & & & & & & \\
\hline & & & & & & & \\
\hline & & & & & & & \\
\hline & & & & & & & \\
\hline & & & & & & & \\
\hline & & $T F, x$ & $T F, y$ & $T F, z$ & & & \\
\hline$y$ & 0.6 & 0.30 & 6.67 & 7.14 & & & \\
\hline$y$ & 0.4 & 0.29 & 7.08 & 6.74 & & & \\
\hline$y$ & 0.1 & 0.19 & 11.39 & 4.79 & & & \\
\hline & & & & & & & \\
\hline & & & & & & & \\
\hline & & & & & & & \\
\hline & & & & & & & \\
\hline & & & & & & & \\
\hline & & & & & & & \\
\hline & & & & & & & \\
\hline & $A \vee G$ & 0.26 & 8.38 & 6.22 & & & \\
\hline
\end{tabular}




\begin{tabular}{|c|c|c|c|c|c|c|c|}
\hline ExDir & ExLeV & $\# 1,(X, M)$ & $\# 2,(Y, M)$ & $\# 3,(Z, M)$ & $\# 4,(X, G)$ & $\# 5,(Y, G)$ & $\# 6,(Z, G)$ \\
\hline & & mic. RMS & mic. RMS & mic. RMS & mic. RMS & mic. FMS & mic. RMS \\
\hline$x$ & 0.6 & 1.0910 & 0.6486 & 0.1305 & 0.5608 & 0.2849 & 0.0300 \\
\hline$x$ & 0.4 & 0.7096 & 0.4184 & 0.0821 & 0.3635 & 0.1835 & 0.0193 \\
\hline$x$ & 0.1 & 0.1516 & 0.0927 & 0.02331 & 0.0773 & 0.0423 & 0.0109 \\
\hline$y$ & 0.6 & 0.4812 & 0.28931 & 0.06181 & 0.2461 & 0.1365 & 0.0190 \\
\hline$y$ & 0.4 & 0.3799 & 0.2220 & 0.0497 & 0.1951 & 0.1038 & 0.0176 \\
\hline$y$ & 0.1 & 0.1331 & 0.08111 & 0.0177 & 0.0689 & 0.0377 & 0.0099 \\
\hline$z$ & 0.6 & 0.1913 & 0.1145 & 0.0216 & 0.0977 & 0.0515 & 0.0137 \\
\hline$z$ & 0.4 & 0.0780 & 0.05031 & 0.0174 & 0.0409 & 0.0227 & 0.0110 \\
\hline$z$ & 0.1 & 0.0818 & 0.0502 & 0.01251 & 0.0429 & 0.0227 & 0.0071 \\
\hline & + & 1 & 1 & 1 & & & \\
\hline & & & I & & & & \\
\hline & & & $\bar{i}$ & & & & \\
\hline & & 1 & 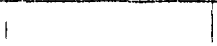 & & & & \\
\hline & 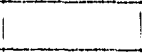 & $!$ & & & & & \\
\hline & & T & 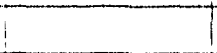 & 1 & & & \\
\hline & & I & 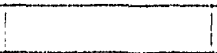 & i & & & \\
\hline & 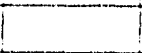 & 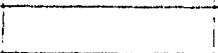 & $\mathrm{i}$ & & & & \\
\hline & & & 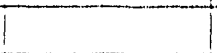 & & & & \\
\hline & & & 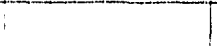 & & & & \\
\hline & & 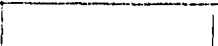 & 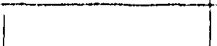 & & & & \\
\hline & & 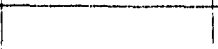 & i & & & & \\
\hline & 1 & 1 & $i$ & & & & \\
\hline
\end{tabular}


RMS, TF, Test $2,10.94 \mathrm{~Hz}$

\begin{tabular}{|c|c|c|c|c|c|c|c|}
\hline EXDir & ExLeV & $\# 1,(X, M)$ & $\# 2,(Y, M)$ & $\# 3,(Z, M)$ & $\# 4,(X, G)$ & $\# 5,(Y, Q)$ & $\# 6,(Z, G)$ \\
\hline & & mic. RMS & mic. RMS & mic. RMS & mic. RMS & mic. RMS & mic. RMS \\
\hline$z$ & 0.6 & 0.1127 & 0.0703 & 0.0221 & 0.0605 & 0.0347 & 0.0138 \\
\hline$z$ & 0.4 & 0.09921 & 0.0668 & 0.0172 & 0.0510 & 0.0347 & 0.0100 \\
\hline$E$ & 0.1 & 0.15081 & 0.0955 & 0.0291 & 0.0773 & 0.0453 & 0.0169 \\
\hline $\bar{x}$ & 0.6 & 0.5375 & 0.3264 & 0.0572 & 0.2812 & 0.1500 & 0.0187 \\
\hline$x$ & 0.4 & $0.4137 !$ & 0.2546 & 0.0416 & 0.2150 & 0.1181 & 0.0137 \\
\hline$x$ & 0.1 & 0.14581 & 0.0916 & 0.0177 & 0.0750 & 0.0426 & 0.0082 \\
\hline$y$ & 0.6 & 0.6033 & 0.3586 & 0.0701 & 0.3153 & 0.1652 & 0.0417 \\
\hline$y$ & 0.4 & 0.3227 & 0.1905 & 0.0417 & 0.1711 & 0.0913 & 0.0223 \\
\hline$y$ & 0.1 & $0.1^{\circ} 012 !$ & 0.0665 & 0.0162 & 0.0522 & 0.0324 & 0.0083 \\
\hline & 1 & 1 & 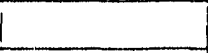 & & & & \\
\hline & 1 & $T F, x$ & $T F, y$ & $T F, z$ & & & \\
\hline$z$ & 0.6 & $1.86 i$ & 2.03 & 1.60 & & & \\
\hline$z$ & 0.4 & 1.951 & 1.93 & 1.72 & & & \\
\hline$z$ & 0.11 & 1.951 & 2.11 & 1.73 & & & \\
\hline $\bar{x}$ & $0.6 i$ & 1.91 & 2.18 & 3.05 & & & \\
\hline$x$ & 0.4 & 1.92 & 2.16 & 3.03 & & & \\
\hline$x$ & 0.1 & 1.94 & 2.15 & 2.17 & & & \\
\hline$y$ & 0.6 & 1.91 & 2.17 & 1.68 & & & \\
\hline$y$ & 0.4 & $1.89 !$ & 2.09 & 1.87 & & & \\
\hline$y$ & 0.1 & 1.941 & 2.05 & 1.96 & & & \\
\hline & 1 & & & & & & \\
\hline & AVG & $1.92 !$ & 2.09 & 2.09 & & & \\
\hline
\end{tabular}


Test 3, AMBIENT, $10.94 \mathrm{~Hz}$

\begin{tabular}{|c|l|r|r|r|}
\hline & \multicolumn{1}{|c|}{ A } & \multicolumn{1}{|c|}{ B } & \multicolumn{1}{c|}{ C } & \multicolumn{1}{c|}{ D } \\
\hline 1 & Location & Amp Gain & MS Volts & RMS microns \\
\hline 2 & $x \mathrm{~m}$ & 10 & 0.0006886 & 0.0842867 \\
\hline 3 & $y \mathrm{~m}$ & 10 & 0.0003246 & 0.05786951 \\
\hline 4 & $z \mathrm{~m}$ & 10 & 0.00001681 & 0.0131692 \\
\hline 5 & $x f$ & 100 & 0.0007143 & 0.00858452 \\
\hline 6 & $y f$ & 100 & 0.0003664 & 0.00614827 \\
\hline 7 & $z f$ & 100 & 0.0002949 & 0.00551586 \\
\hline 8 & & & & \\
\hline 9 & & & & \\
\hline 10 & TFx & 9.81845568 & & \\
\hline 11 & TFy & 9.41231656 & & \\
\hline 12 & TFz & 2.38751699 & & \\
\hline
\end{tabular}


RMS, TF, Test5, 10.94Hz

\begin{tabular}{|c|c|c|c|c|c|c|c|}
\hline EXDir & LEXLov & $\# 1,(X, M)$ & $\# 2,(Y, M)$ & $\# 3,(Z, M)$ & $\# 4_{1}(X, Q)$ & $\# 5,(Y, G)$ & $\# 6,(Z, G)$ \\
\hline & & mlo. RMS & milc. RMS & mlc. RMS & mic. RMS & mic. RMS & mlc. FMS \\
\hline$y$ & 0.6 & 0.6370 & 0.3846 & 0.0754 & 0.1887 & 0.3342 & 0.0219 \\
\hline & & & & & & & 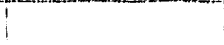 \\
\hline & & & & & & & \\
\hline & & & & & & & $i$ \\
\hline & $!$ & & & & & & $\vdots$ \\
\hline & & & & & & & 1 \\
\hline & & & & & & & T \\
\hline & & & & & & & \\
\hline & & & & & & & 1 \\
\hline & & & & & & & I \\
\hline & & $T F, x$ & TF,Y & $T F, z$ & & & \\
\hline y & 0.6 & 3.38 & 1.15 & 3.44 & & & \\
\hline & & & & & & & I \\
\hline & & & & & & & \\
\hline & & & & & & & I \\
\hline & & & & & & & T \\
\hline & & & & & & & I \\
\hline & & & & & & & $I$ \\
\hline & 1 & & & & & & \\
\hline & 1 & & & & & & \\
\hline & & & & & & & \\
\hline & & & & & & & \\
\hline
\end{tabular}




\begin{tabular}{|c|c|c|c|c|c|c|c|}
\hline EXDir & EXLeV & $\# 1,(X, M)$ & $\# 2,(\overline{Y, M})$ & $\# 3,(Z, M)$ & $\# 4,(X, G)$ & $\# 5,(Y, Q)$ & $\# 6,(2, a)$ \\
\hline & & mlc. FMS & mlo. FMS & mic. RMS & $\mathrm{m} / \mathrm{c}, \mathrm{RMS}$ & Imlc. RMS & mlc. RMS \\
\hline$y$ & 0.6 & 0.5465 & 0.2879 & 0.0777 & 0.14931 & $1 \quad 0.2677$ & 0.0238 \\
\hline$y$ & 0,4 & 0.3938 & 0.2064 & $0.0547 i$ & 0.11001 & 0.1911 & 0.0199 \\
\hline$y$ & 0.1 & 0.1635 & 0.0859 & 0.02861 & 0.0421 & 0.0768 & 0.0194 \\
\hline & & & & 1 & -1 & 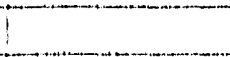 & \\
\hline & & & & 1 & I & & \\
\hline & & & & & & & \\
\hline & & & & & & & \\
\hline & & & & & & & \\
\hline & & & & & & & \\
\hline & & & & & & & \\
\hline & & $T F, x$ & $T F, y$ & $T F, z$ & & | & 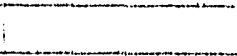 \\
\hline y & 0.6 & 3.66 & 1.08 & 3.26 & & - & $i$ \\
\hline$y$ & 0.4 & 3.58 & 1.08 & 2.74 & & 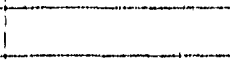 & 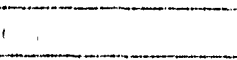 \\
\hline$y$ & 0.1 & 3.89 & 1.12 & 1.48 & & & \\
\hline & & & & & & & \\
\hline & & & & & & & \\
\hline & & & & & & & \\
\hline & & & & & & & \\
\hline & & & & & & & \\
\hline & & & & & & 7 & i \\
\hline & & & & & & & 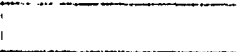 \\
\hline & $\overline{A V G}$ & 3.71 & 1.09 & 2.491 & & 7 & 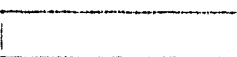 \\
\hline
\end{tabular}


RMS, TF, Test1, $11,72 \mathrm{~Hz}$

\begin{tabular}{|c|c|c|c|c|c|c|c|}
\hline EXDIr & ExLeV & $\# 1,(X, M)$ & $\# 2,(Y, M)$ & $\# 3,(Z, M)$ & $\# 4,(X, G)$ & $\# 5,(Y, G)$ & $\# 6,(Z, Q)$ \\
\hline & & mic. RMS & mic. RMS & mlc. $9 M S$ & mic. RMS & mio. RMS & Imlc. AMS \\
\hline$x$ & 0.6 & 0.2442 & 0.1743 & 0.0222 & 0.1272 & 0.0847 & 0.0111 \\
\hline$x$ & 0.4 & 0.1807 & 0.1326 & 0.0176 & 0.0925 & 0.0646 & 0.0074 \\
\hline$x$ & 0.1 & 0.0431 & 0.0335 & 0.0093 & 0.02101 & 0.0171 & 0.0059 \\
\hline$y$ & 0.6 & 0.1193 & 0.0893 & 0.0133 & 0.0607 & 0.0524 & 0.0068 \\
\hline$y$ & 0.4 & 0.1051 & 0.0765 & 0.0111 & 0.0525 & 0.04391 & 0.0066 \\
\hline$y$ & 0.1 & 0.0382 & 0.0306 & 0.0061 & $0.0176 i$ & 0.0156 & 0.0057 \\
\hline$z$ & 0.6 & 0.0455 & 0.0343 & 0.00761 & 0.02111 & 0.01781 & 0.0069 \\
\hline$z$ & 0.41 & 0.0266 & 0.0169 & 0.01001 & 0.0137 & 0.00751 & 0.0073 \\
\hline$z$ & 0.1 & 0.0260 & 0.0197 & 0.0045 & 0.0129 & 0.0095 & 0.0030 \\
\hline & & & 5 & & & & \\
\hline & & & 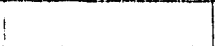 & & & T & \\
\hline & & 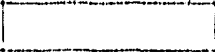 & 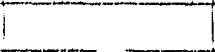 & & & $T$ & \\
\hline & & & 1 & & & 1 & \\
\hline & & & 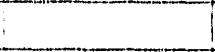 & & & 1 & \\
\hline & & & 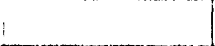 & & & 1 & \\
\hline & & 7 & 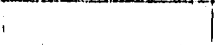 & & & $T$ & \\
\hline & & 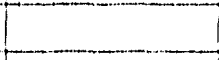 & 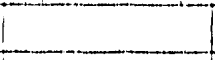 & & & 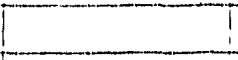 & \\
\hline & & - & I & & & 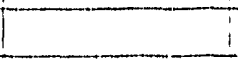 & \\
\hline & & - & I & & & 1 & \\
\hline & & - & I & & & & \\
\hline & & & 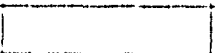 & & & & \\
\hline & & 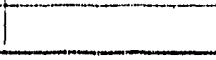 & 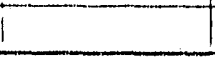 & & & 1 & \\
\hline
\end{tabular}




\begin{tabular}{|c|c|c|c|c|c|c|c|}
\hline EXD/r & r'ExLeV & $\# 1,(X, M)$ & $\# 2,(Y, M)$ & $\# 3,(Z, M)$ & $\# 4,(X, Q)$ & $\# 5,(Y, G)$ & $\# \sigma,(Z, G)$ \\
\hline & 1 & mic. RMS & Imic. RMS & mlc. RMS & mlo. RMS & Imic. RMS & Imlc. RMS \\
\hline$z$ & 0.6 & 0.0357 & 0.0234 & 0.0092 & 0.0205 & 0.0140 & 0.0077 \\
\hline$z$ & 0.4 & $0.0322 !$ & 0.02641 & $0.0066 i$ & 0.0160 & 0.01581 & 0.0058 \\
\hline$\Sigma$ & 0.1 & 0.06981 & 0.0472 & 0.01441 & 0.0357 & 0.0230 & 0.0098 \\
\hline$x$ & 0.6 & $0.1522 !$ & 0.1126 & 0.01681 & 0.0722 & 0.0557 & 0.0098 \\
\hline$x$ & 0.41 & 0.09791 & 0.06591 & 0.01081 & 0.0490 & 0.0327 & 0.0078 \\
\hline$x$ & 0.1 & 0.03791 & 0.03101 & 0.0061 & 0.0174 & $0.0156 i$ & 0.0039 \\
\hline$y$ & 0.61 & 0.1991 & 0.12531 & $0.0317^{\prime}$ & 0.1190 & 0.0588 & 0.0240 \\
\hline$y$ & 0.41 & 0.1261 & 0.07101 & $0.0232 !$ & 0.0770 & 0.0316 & 0.0164 \\
\hline$y$ & 0.1 & 0.03631 & 0.02311 & 0.00711 & 0.0213 & 0.01081 & 0.0047 \\
\hline & $I$ & & & & & & \\
\hline & 1 & $T F, x$ & $T F, Y$ & $T F, z$ & & & \\
\hline$z$ & 0.61 & 1.741 & 1.671 & 1.201 & & & \\
\hline$z$ & 0.4 & $2.02 !$ & 1.681 & 1.15 & & & \\
\hline$z$ & 0.1 & $1.96 i$ & 2.061 & $1.48 i$ & & & \\
\hline$x$ & 0.61 & 2.11 & $2.02 !$ & 1.71 & & & \\
\hline$x$ & 0.4 & 2.001 & 2.02 & $1.38 !$ & & & \\
\hline$x$ & 0.11 & 2.181 & 1.98 & 1.55 & & & \\
\hline$y$ & 0.6 & $1.67 !$ & 2.13 & $1.32 !$ & & & \\
\hline$y$ & 0.4 & 1.64 & $2.25 i$ & 1.41 & & & \\
\hline$y$ & 0.1 & 1.701 & 2.14 & 1.51 & & & \\
\hline & & & & & & & \\
\hline & IAVG & $1.89 !$ & 1.99 & 1.41 & & & \\
\hline
\end{tabular}


Test 3, AMBIENT,11.72 Hz

\begin{tabular}{|c|c|c|c|c|c|}
\hline & A & B & & $\mathrm{C}$ & $D$ \\
\hline 1 & Location & Amp Gain & & MS Volts & RMS miciuns \\
\hline 2 & $x \mathrm{~m}$ & $!$ & 10 & 0.000121 & 0.035332 \\
\hline 3 & $y m$ & ! & $10:$ & 0.00004754 & 0.0221465 \\
\hline 4 & $2 m$ & $!$ & 10 & 0.00000412 & 0.00651965 \\
\hline 5 & $x f$ & 1 & $100:$ & 0.0001451 & 0.00386909 \\
\hline 6 & $y f$ & 1 & 100 & 0.0001019 & 0.00324237 \\
\hline 7 & $2 f$ & $i$ & $100 i$ & 0.0001069 & 0.00332097 \\
\hline 8 & & 1 & 1 & & \\
\hline 9 & & 1 & 1 & & \\
\hline 10 & $T F x$ & 9.131854 & 441 & & \\
\hline 11 & TFy & 6.830342 & $274 !$ & & \\
\hline 12 & $i \mathrm{Fz}$ & 1.963178 & 835 & & \\
\hline
\end{tabular}


RMS, TF,Test5, $11.72 \mathrm{~Hz}$

\begin{tabular}{|c|c|c|c|c|c|c|c|}
\hline EXDir & ExLeV & $\# 1,(X, M)$ & $\# 2,(Y, M)$ & $\# 3,(Z, M)$ & $\# 4,(X, G)$ & $\# 5,(Y, G)$ & $\# 6,(Z, G)$ \\
\hline & & mic. RMS & mic. RMS & Imic. RMS & mic. RMS & mic. RMS & Imic. RMS \\
\hline$y$ & 0.6 & 0.1652 & 0.1057 & 0.0148 & 0.0667 & 0.0902 & 0.0068 \\
\hline & & & & & & & \\
\hline & & & & & & & 1 \\
\hline & & & & 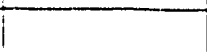 & & & ! \\
\hline & & & 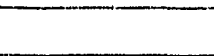 & 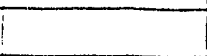 & & & 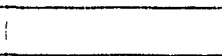 \\
\hline & 1 & & 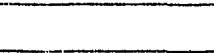 & 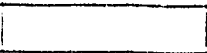 & & & 1 \\
\hline & & & 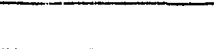 & 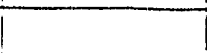 & & & T \\
\hline & & & & & & & I \\
\hline & & & & & & & \\
\hline & & & & & & & \\
\hline & & $T F, x$ & $T F, y$ & $T F, z$ & & & $i$ \\
\hline$y$ & 0.6 & 2.48 & 1.17 & 2.18 & & & I \\
\hline & 1 & & & & & 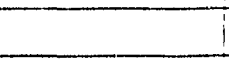 & \\
\hline & 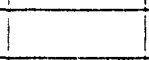 & & & & & & \\
\hline & & & & & & & \\
\hline & 1 & & & & & & \\
\hline & & & & & & & \\
\hline & & & & & & & \\
\hline & & & & & & & \\
\hline & T & & & & & & \\
\hline & & & & & &. & \\
\hline & T & & & & & & \\
\hline
\end{tabular}


RMS, TF, Test6,11.72Hz

\begin{tabular}{|c|c|c|c|c|c|c|c|}
\hline EXDir & ExLeV & $\# 1,(X, M)$ & $\# 2,(Y, M)$ & $\# 3,(Z, M)$ & $\# 4,(X, G)$ & $\# 5,(Y, G)$ & $\# 6,(Z, G)$ \\
\hline & & mic. RMS & mic. RMS & mic. RMS & mic. RMS & mic. RMS & mic. RMS \\
\hline$y$ & 0.6 & 0.0938 & 0.0707 & 0.0168 & 0.0448 & 0.0483 & 0.0104 \\
\hline$y$ & 0.4 & 0.0918 & 0.0521 & 0.0132 & 0.0396 & 0.0448 & 0.0077 \\
\hline \multirow[t]{9}{*}{$y$} & 0.1 & 0.0250 & 0.0192 & 0.0105 & 0.0109 & 0.0132 & 0.0071 \\
\hline & & 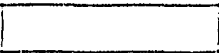 & 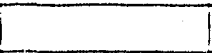 & & & & \\
\hline & & 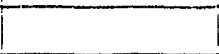 & 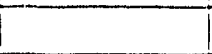 & & & & \\
\hline & & 1 & 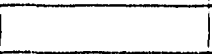 & & & & \\
\hline & & 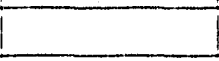 & 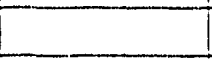 & & & & \\
\hline & & T & 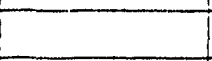 & & & & \\
\hline & & & & & & & \\
\hline & & & & & & & \\
\hline & & $T F, x$ & $T F, y$ & $T F, z$ & & & \\
\hline y & 0.6 & 2.10 & 1.46 & 1.611 & & & \\
\hline$y$ & 0.4 & 2.32 & 1.16 & 1.71 & & & \\
\hline$y$ & 0.1 & 2.29 & 1.46 & 1.47 & & & \\
\hline & & & & & & & \\
\hline & & & & & & & \\
\hline & & & & & & & \\
\hline & & & & & & & \\
\hline & & & & & & & \\
\hline & & & & & & & \\
\hline & & & & & & & \\
\hline & $A \vee G$ & 2.24 & 1.36 & 1.601 & & & \\
\hline
\end{tabular}


RMS, TF, Test1, 21.09Hz

\begin{tabular}{|c|c|c|c|c|c|c|c|}
\hline EXDir & ExLeV & $\# 1,(X, M)$ & $\# 2,(Y, M)$ & $\# 3,(Z, M)$ & $\# 4,(X, G)$ & $\# 5,(Y, G)$ & $\# 6,(Z, G)$ \\
\hline & & mic. RMS & mic. RMS & mic. RMS & mic. RMS & mic. RMS & mic. RMS \\
\hline$x$ & 0.6 & 0.8374 & 0.6878 & 0.2484 & 0.1691 & 0.2191 & 0.0689 \\
\hline$x$ & 0.4 & 0.5009 & 0.4131 & 0.1464 & 0.0957 & 0.1304 & 0.0408 \\
\hline$x$ & 0.1 & 0.09731 & 0.08581 & 0.03101 & 0.0190 & 0.0263 & 0.0091 \\
\hline$y$ & 0.6 & 0.6537 & 0.4321 & 0.1852 & 0.1264 & 0.1342 & 0.0449 \\
\hline$y$ & 0.4 & 0.4228 & 0.28781 & 0.1210 & 0.0806 & 0.0883 & 0.0335 \\
\hline$y$ & 0.1 & 0.1089 & 0.0732 & 0.0341 & 0.0211 & 0.0236 & 0.0088 \\
\hline$z$ & 0.6 & 0.2278 & 0.13861 & 0.0805 & 0.0456 & 0.0367 & 0.0187 \\
\hline$z$ & 0.4 & 0.1623 & 0.0976 & 0.0591 & 0.0325 & 0.0260 & 0.0141 \\
\hline$z$ & 0.1 & 0.0453 & 0.0286 & 0.0149 & 0.0091 & 0.0084 & 0.0054 \\
\hline & & & & & & & \\
\hline & & & & & & & \\
\hline & & & & & & & \\
\hline & & & & & & & \\
\hline & i & L & & & & & \\
\hline & & & & & & & \\
\hline & & & & & & & \\
\hline & & & & & & & \\
\hline & & & & & & & \\
\hline & & & & & & & \\
\hline & & & & & & & \\
\hline & & & & & & & \\
\hline & & & & & & & \\
\hline
\end{tabular}


RMS, TF, Test2, $20.09 \mathrm{~Hz}$

\begin{tabular}{|c|c|c|c|c|c|c|c|}
\hline ExDir & ExLev & $\# 1,(X, M)$ & $\# 2,(Y, M)$ & $\# 3,(Z, M)$ & $\# 4,(X, G)$ & $\# 5,(Y, G)$ & $\# 6,(Z, G)$ \\
\hline & & mic. RMS & mic. RMS & mic. RMS & mic. RMS & mic. RMS & mic. RMS \\
\hline$z$ & 0.6 & 0.27711 & 0.1951 & 0.0795 & 0.0478 & 0.0396 & 0.0164 \\
\hline$z$ & 0.4 & 0.18081 & 0.1258 & 0.0571 & 0.0315 & 0.02601 & 0.0128 \\
\hline$z$ & 0.11 & 0.04891 & 0.0325 & 0.01561 & 0.0088 & 0.00781 & 0.0066 \\
\hline$x$ & 0.61 & 0.3974 & 0.2105 & $0.1112 !$ & 0.0640 & 0.05291 & 0.0087 \\
\hline$x$ & 0.4 & 0.2474 & 0.1341 & 0.07391 & 0.0396 & 0.03281 & 0.0074 \\
\hline$x$ & 0.1 & 0.05991 & 0.0338 & 0.0198 & 0.0097 & 0.0089 & 0.0052 \\
\hline$y$ & 0.6 & 0.7167 & 0.5646 & 0.21011 & 0.1275 & 0.11381 & 0.0445 \\
\hline$y$ & 0.41 & $0.4445 i$ & 0.3499 & 0.12601 & 0.0791 & 0.07161 & 0.0227 \\
\hline \multirow[t]{3}{*}{ y } & 0.1 & 0.0996 & 0.0805 & 0.02931 & 0.0179 & 0.01681 & 0.0062 \\
\hline & & & & & & & \\
\hline & & TF, $x$ & $T F, y$ & $T F, z$ & & & \\
\hline$z$ & 0.6 & 5.80 & 4.93 & 4.85 & & & \\
\hline 2 & 0.4 & $5.74 !$ & 4.84 & 4.461 & & & \\
\hline$z$ & 0.1 & 5.581 & 4.151 & 2.36 & & & \\
\hline$x$ & 0.6 & 6.21 & 3.98 & $12.75 i$ & & & \\
\hline$x$ & 0.4 & 6.24 & 4.09 & 10.011 & & & \\
\hline$x$ & 0.1 & 6.15 & 3.81 & 3.801 & & & \\
\hline$y$ & 0.61 & $5.62 !$ & 4.96 & $4.72 !$ & & & \\
\hline$y$ & 0.41 & $5.62 !$ & 4.88 & 5.54 & & & \\
\hline \multirow[t]{3}{*}{ y } & $0.1 !$ & 5.56 & 4.80 & 4.731 & & & \\
\hline & & & & & & & \\
\hline & AVG & 5.84 & 4.49 & 5.921 & & & \\
\hline
\end{tabular}


Test 3, AMBIENT,21.09 Hz

\begin{tabular}{|c|c|c|c|c|}
\hline & A & $B$ & $c$ & $D$ \\
\hline 1 & Location & Ainp Gain & MS Volts & RMS microns \\
\hline 2 & $x m$ & 10 & 0.00001017 & 0.01024321 \\
\hline 3 & $y m$ & 101 & 0.000008531 & 0.00938102 \\
\hline 4 & $z m$ & 10 & $4.447 E .061$ & 0.00677344 \\
\hline 5 & $x f$ & 100 & 0.00005661 & 0.0024167 \\
\hline 6 & $y f$ & 100 & 0.00002915 & 0.00173418 \\
\hline 7 & $2 f$ & 100 & 0.000074281 & 0.00276829 \\
\hline 8 & & & & \\
\hline 9 & & & & \\
\hline 10 & TFx & 4.2385167 & & \\
\hline 11 & TFy & 5.40947647 & & \\
\hline 12 & $T F z$ & 2.44679519 & & \\
\hline
\end{tabular}


RMS, TF,Test5,21.09Hz

\begin{tabular}{|c|c|c|c|c|c|c|c|}
\hline EXDir & ExLeV & $\# 1,(X, M)$ & $\# 2,(Y, M)$ & $\# 3,(Z, M)$ & $\# 4,(X, G)$ & $\# 5,(Y, G)$ & $\# 6,(Z, G)$ \\
\hline & & mic. RMS & mic. RMS & mic. RMS & mic. RMS & mic. RMS & mlc. RMS \\
\hline$y$ & 0.6 & 0.1025 & 0.0938 & 0.0678 & 0.2417 & 0.1735 & 0.2769 \\
\hline & & & & & & & \\
\hline & & & & & & & \\
\hline & & & & & & & \\
\hline & | & 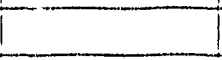 & & & & & \\
\hline & & & & & & & \\
\hline & & & & & & & \\
\hline & & & & & &. & \\
\hline & & & & & & & \\
\hline & & & & & & & \\
\hline & & $T F, x$ & TF,Y & $T F, z$ & & & \\
\hline$y$ & 0.6 & 0.42 & 0.54 & 0.24 & & & \\
\hline & & & & & & & \\
\hline & & & & & & & \\
\hline & & & & & & & \\
\hline & & & & & & & \\
\hline & & & & & & & \\
\hline & & & & & & & \\
\hline & & & & & & & \\
\hline & & & & & & & \\
\hline & & & & & & & \\
\hline & & & & & & & \\
\hline
\end{tabular}


RMS, TF, Test6, $21.09 \mathrm{~Hz}$

\begin{tabular}{|c|c|c|c|c|c|c|c|}
\hline EXDir & ExLev & $\# 1,(X, M)$ & $\# 2,(Y, M)$ & $\# 3,(Z, M)$ & $\# 4,(X, G)$ & $\# 5,(Y, G)$ & $\# G,(Z, G)$ \\
\hline & & mic. FMS & mic. RMS & mic. RMS & mic. RMS & mic. RMS & Imic. RMS \\
\hline y & 0.6 & 0.2233 & 0.3715 & 0.0591 & 0.1344 & 0.0469 & 0.0551 \\
\hline$y$ & 0.4 & 0.1762 & 0.2281 & 0.0530 & 0.0830 & 0.03791 & 0.0376 \\
\hline$y$ & 0.1 & 0.0649 & 0.0574 & 0.0234 & 0.0213 & 0.01541 & 0.0145 \\
\hline & & & & & & & \\
\hline & & & & & & & \\
\hline & & & & & & & \\
\hline & & & & & & & \\
\hline & & & & & & & \\
\hline & & & & & & & \\
\hline & & & & & & & \\
\hline & & $T F, x$ & $T F, Y$ & $T F, z$ & & & \\
\hline y & 0.6 & 1.66 & 7.92 & 1.07 & & & \\
\hline$y$ & 0.4 & 2.12 & 6.02 & 1.41 & & & \\
\hline$y$ & 0.1 & 3.05 & 3.72 & 1.61 & & & \\
\hline & & & & & & & \\
\hline & & & & & & & \\
\hline & & & & & & & \\
\hline & & & & & & & \\
\hline & & & & & & & \\
\hline & & & & & & & \\
\hline & & & & & & & \\
\hline & AVG & 2.281 & 5.88 & 1.36 & & & \\
\hline
\end{tabular}


RMS, TF, Test1,23.63Hz

\begin{tabular}{|c|c|c|c|c|c|c|c|}
\hline ExDir & ExLEV & $\# 1,(X, M)$ & $\# 2,(Y, M)$ & $\# 3,(Z, M)$ & $\# 4,(X, G)$ & $\# 5,(Y, G)$ & $\# 6,(Z, G)$ \\
\hline & & mic. RMS & mic. RMS & mic. RMS & mic. RMS & mic. RMS & mic. RMS \\
\hline$x$ & 0.6 & 0.2841 & 0.2222 & 0.0643 & 0.0347 & 0.0584 & 0.0212 \\
\hline$x$ & 0.4 & 0.1868 & 0.0858 & 0.0425 & 0.0214 & 0.0231 & 0.0116 \\
\hline$\underline{x}$ & 0.1 & 0.0468 & 0.01301 & 0.0113 & 0.0060 & 0.00641 & 0.0049 \\
\hline$y$ & 0.6 & 0.3003 & 2.05931 & 0.0578 & 0.0422 & 0.5267 & 0.1999 \\
\hline$y$ & 0.4 & 0.1791 & 1.2527 & 0.03901 & 0.0290 & 0.3169 & 0.1453 \\
\hline$y$ & 0.1 & 0.0500 & 0.3418 & 0.0119 & 0.0085 & 0.0884 & 0.0391 \\
\hline$z$ & 0.6 & 0.0938 & 0.2966 & 0.0406 & 0.0113 & 0.0766 & 0.0287 \\
\hline$z$ & 0.4 & 0.0734 & 0.2252 & 0.0315 & 0.0101 & 0.0578 & 0.0242 \\
\hline$z$ & 0.1 & 0.0204 & 0.0808 & 0.0107 & 0.0030 & 0.0209 & 0.0099 \\
\hline & - & i & 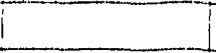 & & & & \\
\hline & & & 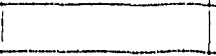 & & & & \\
\hline & & & 1 & & & & \\
\hline & & & i & & & & \\
\hline & & & & & & & \\
\hline & & & & & & & \\
\hline & & $I$ & & & & & \\
\hline & & & & & & & \\
\hline & & & & & & & \\
\hline & & & & & & & \\
\hline & & & & & & & \\
\hline & & & & & & & \\
\hline & & & & & & & \\
\hline
\end{tabular}


RMS, TF, Test2,23.63Hz

\begin{tabular}{|c|c|c|c|c|c|c|c|}
\hline EXDir & ExLeV & $\# 1,(X, M)$ & $\# 2,(Y, M)$ & $\# 3,(Z, M)$ & $\# 4,(X, G)$ & $\# 5,(Y, Q)$ & $\# 6,(Z, G)$ \\
\hline & & mlc. RMS & inlc. RMS & mic. FMS & mic. RMS & mlc. FMS & mlc. RMS \\
\hline$z$ & 0.6 & 0.0559 & 0.2267 & 0.0257 & 0.0092 & 0.0501 & 0.0197 \\
\hline$z$ & 0.4 & 0.0571 & 0.1898 & 0.0185 & 0.0087 & 0.0425 & 0.0168 \\
\hline$z$ & 0.1 & 0.0253 & 0.0861 & 0.0084 & 0.0041 & 0.0196 & 0.0111 \\
\hline$x$ & 0.6 & 0.0646 & 0.0933 & 0.0118 & 0.0165 & 0.0202 & 0.0088 \\
\hline$x$ & 0.4 & 0.0453 & 0.0546 & 0.0092 & 0.0122 & 0.0125 & 0.0058 \\
\hline$x$ & 0.1 & 0.0125 & 0.0185 & 0.0050 & 0.0032 & 0.0048 & 0.0048 \\
\hline$y$ & 0.6 & 0.3814 & 1.9203 & 0.0475 & 0.0417 & 0.4547 & 0.1952 \\
\hline$y$ & 0.4 & 0.2260 & 0.9993 & 0.0294 & 0.0265 & 0.2398 & 0.1138 \\
\hline \multirow[t]{3}{*}{$y$} & 0.1 & 0.0520 & 0.2146 & 0.0083 & 0.0056 & 0.0497 & 0.0237 \\
\hline & & & & & & & \\
\hline & & $T F, x$ & $T F, Y$ & $T F, z$ & & & \\
\hline$z$ & 0,6 & 6.08 & 4.52 & 1.30 & & & \\
\hline$z$ & 0.4 & 0.58 & 4.47 & 1.10 & & & \\
\hline$z$ & 0.1 & 6.10 & 4.39 & 0.76 & & & \\
\hline$x$ & 0.6 & 3.92 & 4.62 & 1.34 & & & \\
\hline$x$ & 0.4 & 3.73 & 4.38 & 1.58 & & & \\
\hline$x$ & 0.1 & 3.85 & 3.89 & 1.04 & & & \\
\hline$y$ & 0.6 & 9.14 & 4.22 & 0.24 & & & \\
\hline$y$ & 0.4 & 8.53 & 4.17 & 0.26 & & & \\
\hline \multirow[t]{3}{*}{$y$} & 0.1 & 9.30 & 4.32 & 0.35 & & & \\
\hline & & & & & & & \\
\hline & $A \vee G$ & 6.36 & 4.33 & 0.89 & & & \\
\hline
\end{tabular}


Test 3, AMBIENT,23.53 Hz

\begin{tabular}{|c|c|c|c|c|}
\hline & A & B & C & $D$ \\
\hline 1 & Location & Amp Gain & IMS Volts & AMS microns \\
\hline 2 & $x r n$ & 10 & $1 \quad 1.158 \mathrm{E} .06$ & 0.00345645 \\
\hline 3 & $y \mathrm{~m}$ & 10 & 0.00000846 & 0.00934245 \\
\hline 4 & $2 m$ & 10 & $1.299 \mathrm{E} .06$ & 0.00366083 \\
\hline 5 & $x f$ & 100 & 0.00003658 & 0.00194266 \\
\hline 6 & yf & 100 & 0.0000142 & 0.00121037 \\
\hline 7 & $z f$ & 100 & 0.00002892 & 0.00172733 \\
\hline 8 & & & & \\
\hline 9 & & & & \\
\hline 10 & TFx & 1.77923138 & & \\
\hline 11 & TFy & 7.71864397 & & \\
\hline 12 & $T F_{2}$ & 2.11936341 & & \\
\hline
\end{tabular}


RMS, TF, TESt5, 23.63Hz

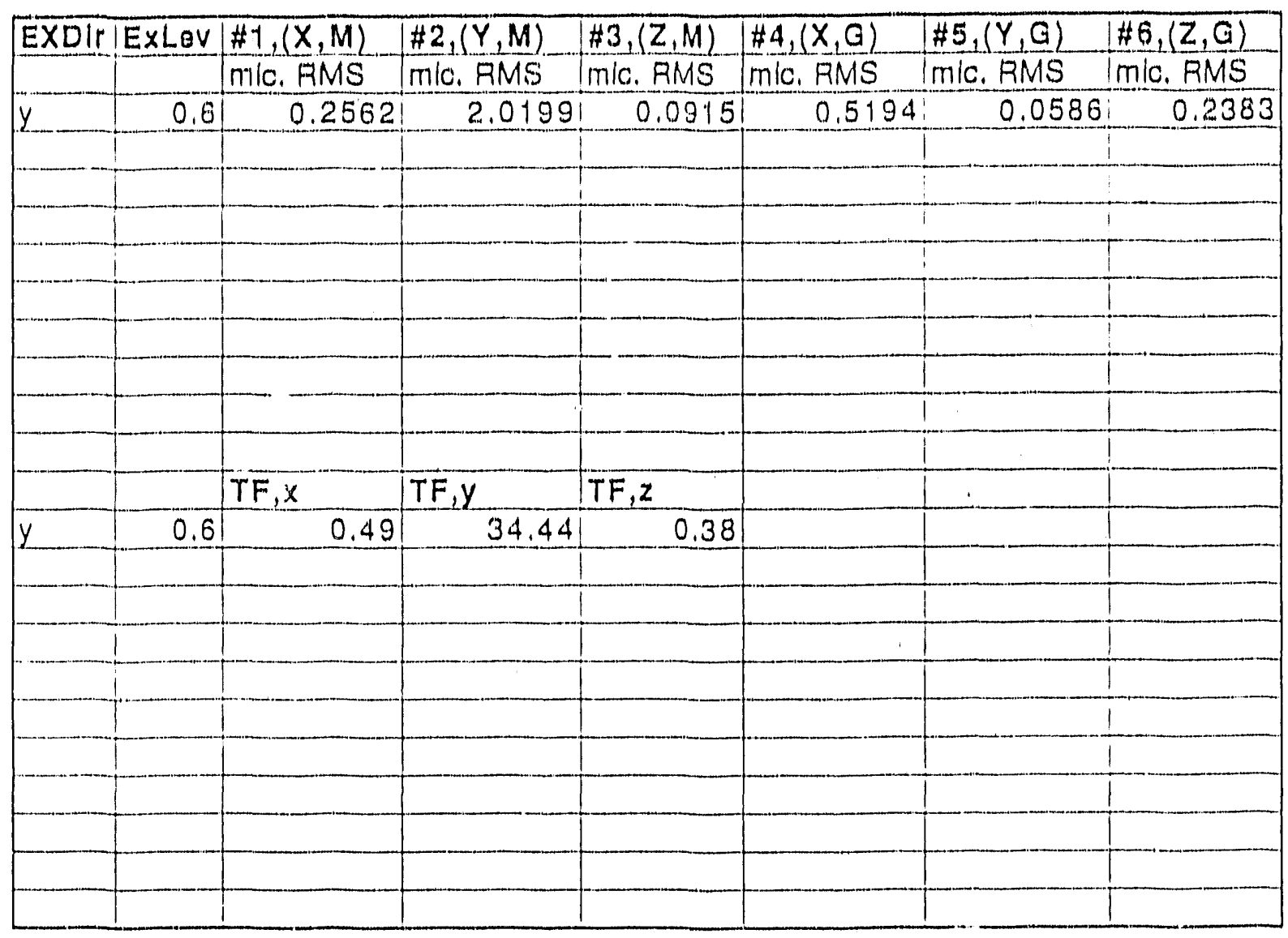


RMS, TF, Test6,23.63Hz

\begin{tabular}{|c|c|c|c|c|c|c|c|}
\hline EXD/r & ExLeV & $\# 1,(X, M)$ & $\# 2,(Y, M)$ & $\# 3,(Z, M)$ & $\# 4,(X, G)$ & $\# 5,(Y, G)$ & $\# 6,(Z, G)$ \\
\hline & & mlc. RMS & mic. RMS & mlc. RMS & mlc. FMS & mic. RMS & mic. RMS \\
\hline$y$ & 0.6 & 0.1022 & 2.1337 & 0.1476 & 0.5669 & 0.0428 & 0.2977 \\
\hline$y$ & 0.4 & 0.06801 & 1.6014 & 0.0781 & 0.42831 & 0.0235 & 0.1827 \\
\hline$y$ & 0.1 & 0.0222 & 0.3440 & 0.0246 & 0.0942 & 0.0156 & 0.0456 \\
\hline & & & & & & & \\
\hline & & & & & & & \\
\hline & & & & & & & \\
\hline & & & & & & & \\
\hline & & $\therefore$ & & & . & & \\
\hline & & & & & & & \\
\hline & & & & & & & \\
\hline & & $\mathrm{TF}_{1} \mathrm{x}$ & $T F, y$ & $T F, z$ & & & \\
\hline$y$ & 0.6 & 0.181 & 49.87 & 0.50 & & & \\
\hline$y$ & 0.4 & 0.16 & 68.06 & 0.43 & & & \\
\hline$y$ & 0.1 & 0.24 & 21.99 & 0.54 & & & \\
\hline & & & & & & & \\
\hline & & & & & & & \\
\hline & & & & & & & \\
\hline & & & & & & & \\
\hline & & & & & & & \\
\hline & & & & & & & \\
\hline & & & & & & & \\
\hline & $A \vee G$ & 0.19 & 46.64 & 0.49 & & & \\
\hline
\end{tabular}


RMS, TF, Test1, 32.42Hz

\begin{tabular}{|c|c|c|c|c|c|c|c|}
\hline EXDir & ExLeV & $\# 1,(X, M)$ & $\# 2,(Y, M)$ & $\# 3,(Z, M)$ & $\# 4,(X, G)$ & $\# 5,(Y, Q)$ & $\# 6,(2, G)$ \\
\hline & & mla. RMS & mlc. RMS & mic. RMS & mic. RMS & mic. FMS & mlo. RMS \\
\hline$x$ & 0.6 & 0.0596 & 0.0348 & 0.0939 & 0.0274 & 0.0174 & 0.0514 \\
\hline $\bar{x}$ & 0.4 & 0.0380 & 0.0219 & 0.0557 & 0.0155 & 0.0093 & 0.0304 \\
\hline $\bar{x}$ & 0.1 & 0.0070 & 0.0058 & 0.0185 & 0.0038 & 0.0031 & 0.0098 \\
\hline$y$ & 0.6 & 0.0204 & 0.1659 & 0.0259 & 0.0107 & 0.0292 & 0.0292 \\
\hline$y$ & 0.4 & 0.0131 & 0.1051 & 0.02461 & 0.0095 & 0.0222 & 0.0255 \\
\hline$y$ & 0.1 & 0.0042 & 0.0278 & 0.0155 & 0.0035 & 0.0057 & 0.0092 \\
\hline$z$ & 0.6 & 0.0349 & 0.0404 & 0.1880 & 0.0294 & 0.0103 & 0.0913 \\
\hline$z$ & 0.4 & 0.0237 & 0.0280 & 0.1133 & 0.0191 & 0.0066 & 0.0542 \\
\hline$z$ & 0.1 & 0.0067 & 0.0074 & 0.0316 & 0.0049 & 0.0037 & 0.0157 \\
\hline & & & & & & & \\
\hline & & & & & & & \\
\hline & & & & & & & \\
\hline & & & & & & & \\
\hline & & & & & & & \\
\hline & & & & & & & \\
\hline & & & & & & & \\
\hline & & & & & & & \\
\hline & & & & & & & \\
\hline & & & & & & & \\
\hline & & & & & & & \\
\hline & & & & & & & \\
\hline & & & & & & & \\
\hline
\end{tabular}


FMS, TF, Test2,32.42 Hz

\begin{tabular}{|c|c|c|c|c|c|c|c|}
\hline EXDir & ExLeV & $\# 1,(X, M)$ & $\# 2,(Y, M)$ & $\# 3,(Z, M)$ & $\# 4,(X, G)$ & $\# 5,(Y, G)$ & $\# 6,(2, G)$ \\
\hline & 1 & mic. RMS & mic. RMS & mic. RMS & mic. RMS & mic. RMS & mic. RMS \\
\hline$z$ & 0.61 & 0.0264 & 0.0258 & 0.1558 & 0.0337 & 0.0112 & 0.0762 \\
\hline 2 & 0.41 & 0.0177 & 0.0171 & 0.1002 & 0.0079 & 0.0055 & 0.0490 \\
\hline$z$ & 0.1 & 0.0071 & 0.00681 & 0.03181 & 0.0038 & 0.0219 & 0.0153 \\
\hline$x$ & 0.61 & 0.0185 & 0.0218 & 0.07211 & 0.0163 & 0.0145 & 0.0426 \\
\hline$x$ & 0.4 & 0.0122 & 0.01501 & 0.04871 & $0.0107 !$ & 0.0045 & 0.0286 \\
\hline$x$ & 0.1 & 0.0051 & 0.00431 & 0.01661 & $0.0036 i$ & 0.0064 & 0.0094 \\
\hline$y$ & 0.61 & 0.1136 & 0.06991 & 0.23881 & 0.0457 & 0.0457 & 0.1293 \\
\hline$y$ & 0.4 & 0.08301 & 0.0494 & 0.1784 & 0.0278 & 0.0166 & 0.0956 \\
\hline \multirow[t]{3}{*}{$y$} & 0.1 & 0.0289 & $0.0192 !$ & 0.0717 & 0.0094 & 0.0022 & 0.0375 \\
\hline & $!$ & 1 & & & & & \\
\hline & 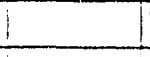 & $T F, x$ & $T F, y$ & $T F, z$ & & & \\
\hline$z$ & 0.6 & 0.781 & 2.291 & 2.04 & & & \\
\hline$z$ & 0.4 & 2.231 & 3.091 & 2.05 & & & \\
\hline$z$ & 0.11 & 1.87 & 0.31 & 2.07 & & & \\
\hline$x$ & 0.61 & 1.13 & 1.501 & 1.69 & & & \\
\hline$x$ & 0.4 & 1.14 & 3.301 & 1.701 & & & \\
\hline$x$ & 0.1 & 1.431 & 0.681 & 1.77 & & & \\
\hline$y$ & 0.6 & $2.49 !$ & 1.531 & 1.85 & & & \\
\hline$y$ & 0.4 & $2.99 !$ & 2.97 & 1.87 & & & \\
\hline$y$ & 0.1 & 3.07 & 8.68 & 1.91 & & & \\
\hline & & & & & & & \\
\hline & $A \vee G$ & 1.901 & 2.701 & 1.881 & & & \\
\hline
\end{tabular}


Test 3, AMBIENT, $32.42 \mathrm{~Hz}$

\begin{tabular}{|c|c|c|c|c|}
\hline & $A$ & $B$ & $\mathrm{C}$ & $D$ \\
\hline 1 & Location & Amp Gain & IMS Volts & IRMS microns \\
\hline 2 & $x \mathrm{~m}$ & 10 & 4.737E.07 & 10.00221069 \\
\hline 3 & $y m$ & 10 & 0.00000163 & 0.00410081 \\
\hline 4 & $2 m$ & 10 & 0.000141 & 0.03814039 \\
\hline 5 & $x f$ & 100 & 10.00001124 & 0.00107686 \\
\hline 6 & $y f$ & 100 & $3.128 E-06$ & 0.00056808 \\
\hline 7 & $z f$ & 100 & 0.00004093 & 0.00205493 \\
\hline 8 & & 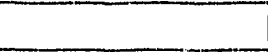 & 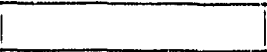 & \\
\hline 9 & & & & \\
\hline 10 & TFx & 2.05290351 & & \\
\hline 11 & $T F y$ & 7.21872388 & & \\
\hline 12 & $T F z$ & 18.5604578 & & \\
\hline
\end{tabular}




\begin{tabular}{|c|c|c|c|c|c|c|c|}
\hline EXDir & ExLeV & $\# 1,(X, M)$ & $\# 2,(Y, M)$ & $\# 3,(Z, M)$ & $\# 4,(X, G)$ & $\# 5,(Y, G)$ & $\# 6,(Z, G)$ \\
\hline & & mic. RMS & mic. RMS & mic. RMS & Imic. RMS & mic. RMS & mic. RMS \\
\hline$y$ & 0.6 & 0.0265 & 0.2180 & 0.0361 & $1 \quad 0.0402$ & 0.0179 & 0.0319 \\
\hline & & & & & & & \\
\hline & & & & & $I$ & & \\
\hline & & & & & 1 & & \\
\hline & & & & & I & & \\
\hline & & & & & & & \\
\hline & & & & & & & \\
\hline & & & & & & & \\
\hline & & & & & & & \\
\hline & & & & & & & \\
\hline & & $T F, x$ & $T F, y$ & $T F, z$ & & & \\
\hline y & 0.6 & 0.66 & 12.16 & 1.131 & & & \\
\hline & & & & & & & \\
\hline & & & & & & & \\
\hline & & & & & & & \\
\hline & & & & & & & \\
\hline & & & & & & & \\
\hline & & & & & & & \\
\hline & & & & & & & \\
\hline & & & & & & & \\
\hline & & & & & & & \\
\hline & & & & & & & \\
\hline
\end{tabular}


RMS, TF, Test6, $32.42 \mathrm{~Hz}$

\begin{tabular}{|c|c|c|c|c|c|c|c|}
\hline EXDir & ExLeV & $\# 1,(X, M)$ & $\# 2,(Y, M)$ & $\# 3,(Z, M)$ & $\# 4,(X, G)$ & $\# 5,(Y, G)$ & $\# 6,(Z, G)$ \\
\hline & & mic. RMS & mic. RMS & mic. RMS & mic. RMS & mic. RMS & mic. RMS \\
\hline$y$ & 0.61 & 0.0225 & 0.2338 & 0.0562 & 0.0394 & 0.0154 & 0.0364 \\
\hline$y$ & 0.41 & 0.0178 & 0.1519 & 0.0399 & 0.0292 & 0.0159 & 0.0274 \\
\hline$y$ & 0.1 & 0.0142 & 0.0443 & 0.0209 & 0.0142 & 0.01301 & 0.0137 \\
\hline & & & & & & & \\
\hline & & & & & & & \\
\hline & & & & & & & \\
\hline & & & & & & & \\
\hline & & & & & & & \\
\hline & & . & & & & & \\
\hline & & & & & & & \\
\hline & & $T F, x$ & $T F, y$ & $T F, z$ & & & \\
\hline$y$ & 0.61 & 0.57 & 15.17 & 1.54 & & & \\
\hline$y$ & 0.4 & 0.61 & 9.58 & 1.45 & & & \\
\hline$y$ & 0.11 & 1.001 & 3.41 & 1.53 & & & \\
\hline & & & & & & & \\
\hline & & & & & & & \\
\hline & & & & & & & \\
\hline & & & & & & & \\
\hline & & & & & & & \\
\hline & & & & & & & \\
\hline & & & & & & & \\
\hline & $A \vee G$ & 0.73 & 9.39 & 1.51 & & & \\
\hline
\end{tabular}


Appendix D

Impulse Excitation Plots for Phase I Tests (Test 7) 

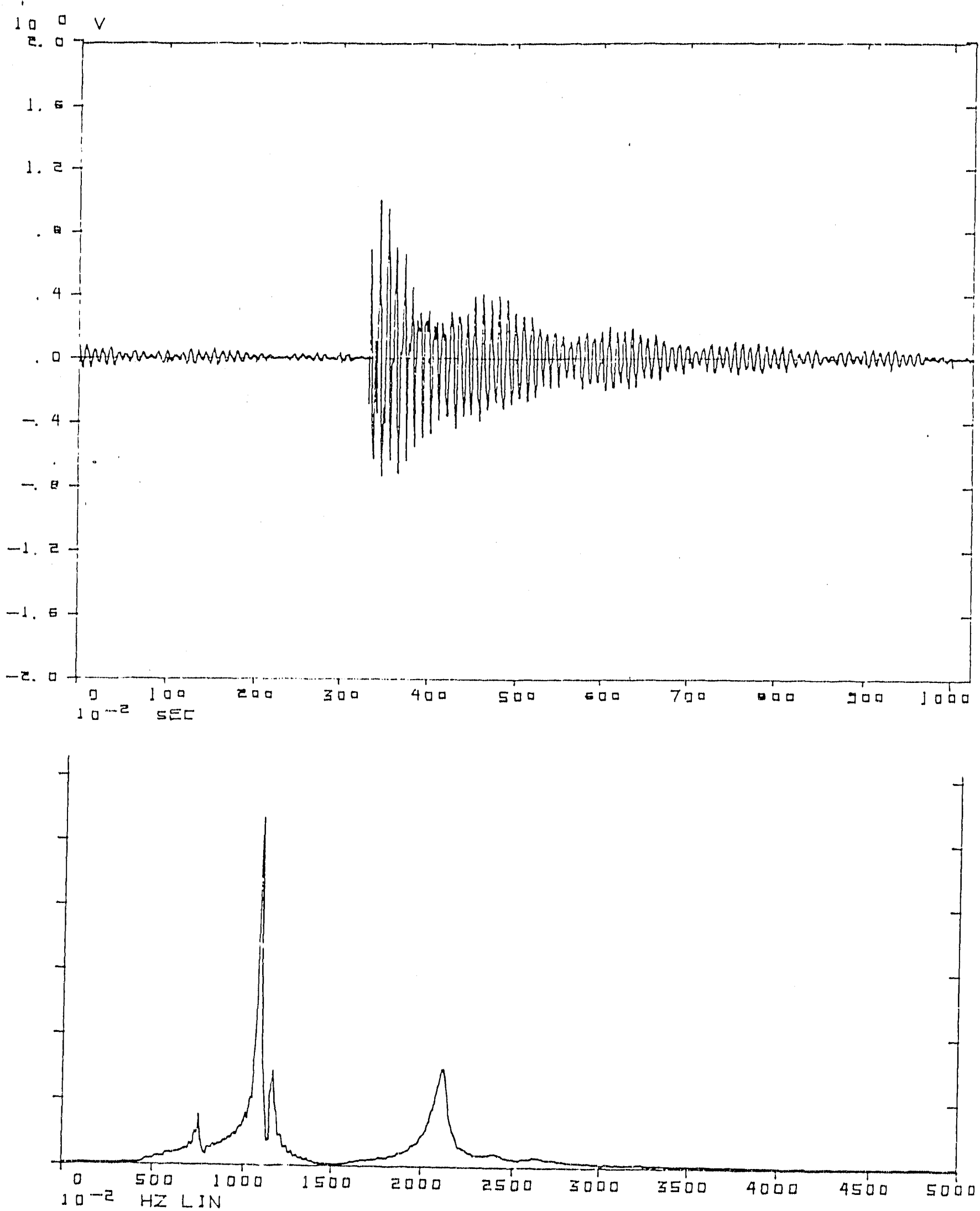

Impulse Excitation on Magnet in $X$ Direction

Magnet Response in $X$ Direction

Top-Real Time, Bottern-Frequency 

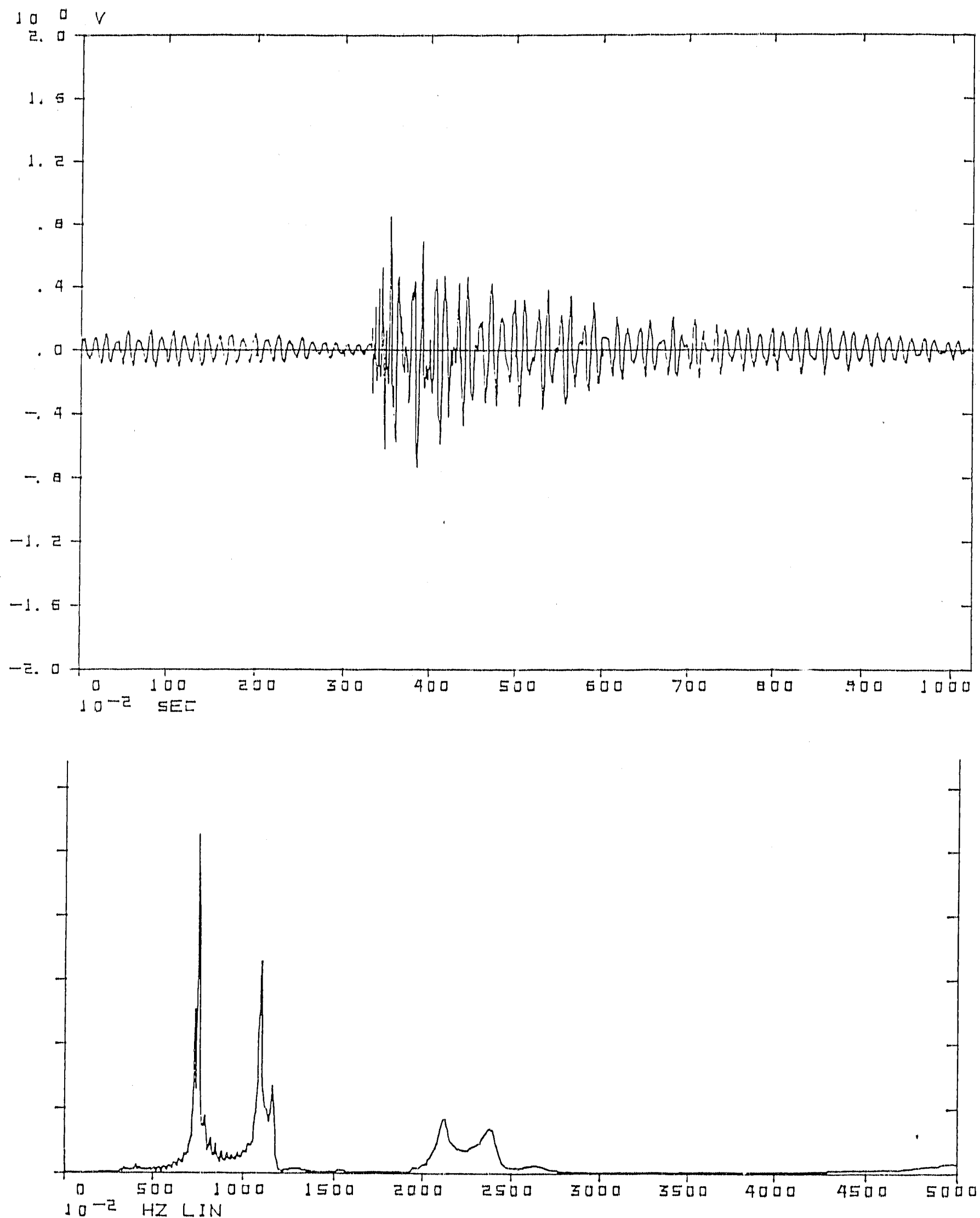

Impulse Excitation on Magnet in $X$ Direction Magnet Response in $Y$ Direction

Top-Real Time, Bottem-Frequency 


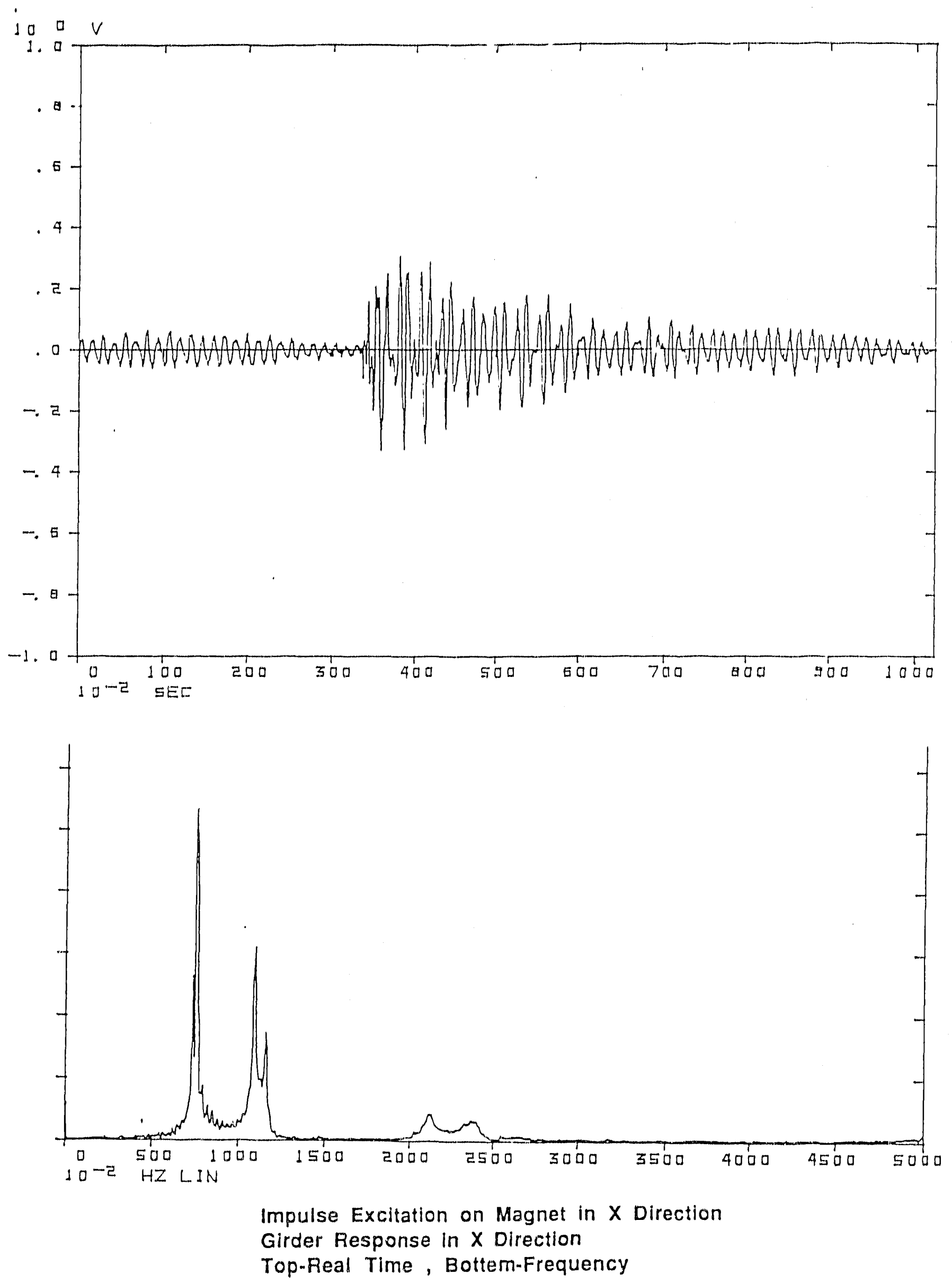



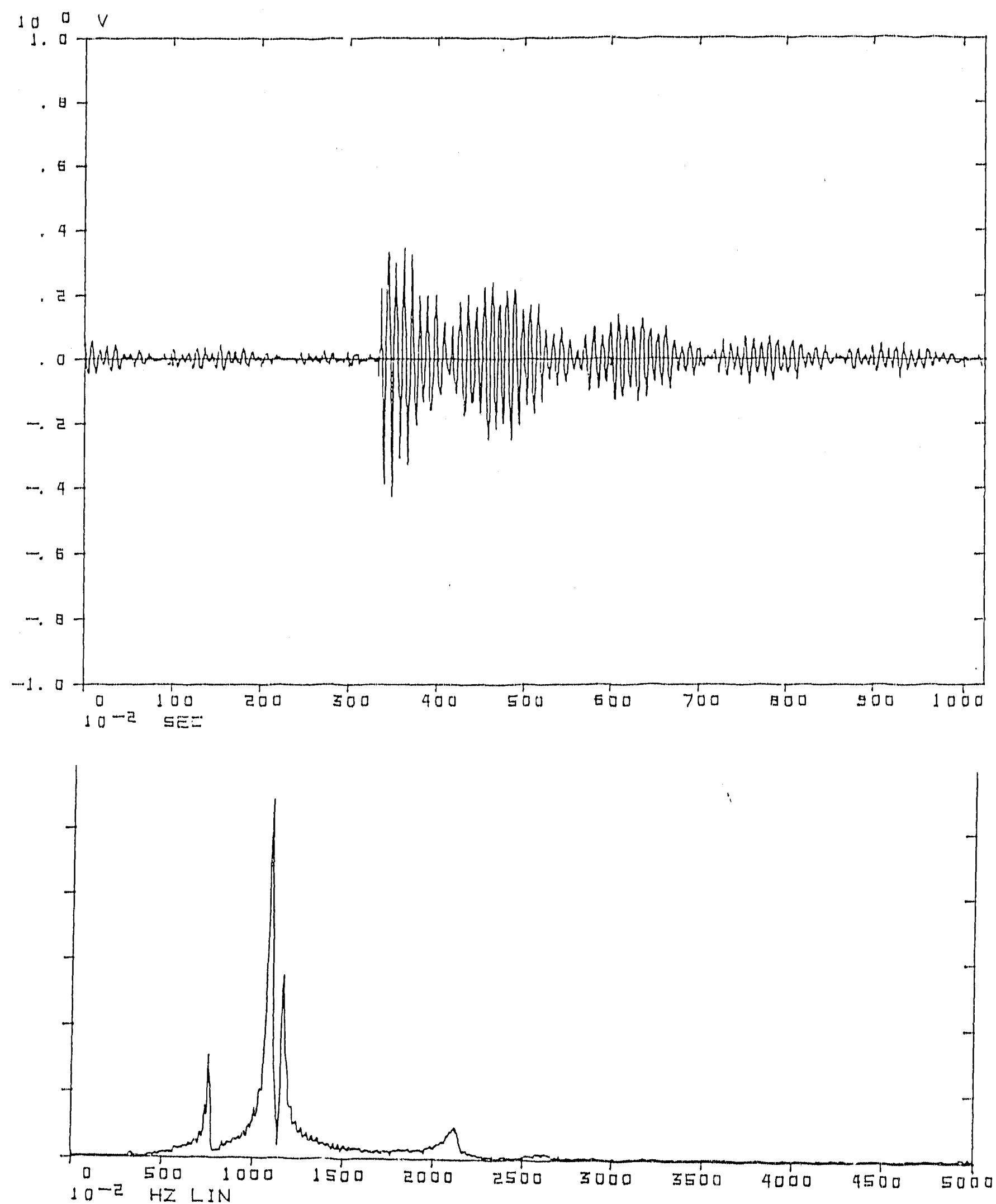

Impulse Excitation on Magnet in $X$ Direction

Girder Response in $Y$ Direction

rop-Real Time, Bottem-Frequency 

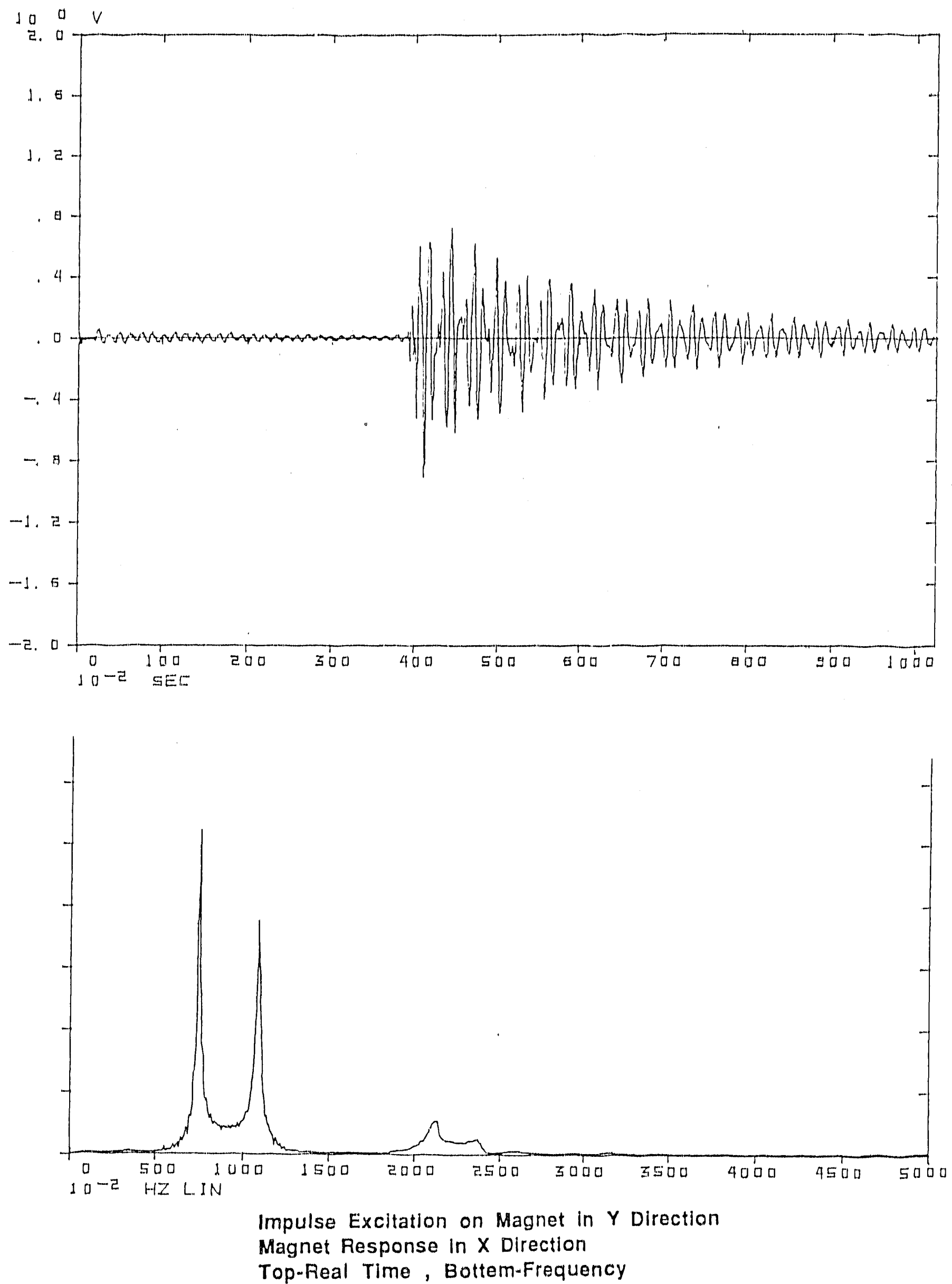

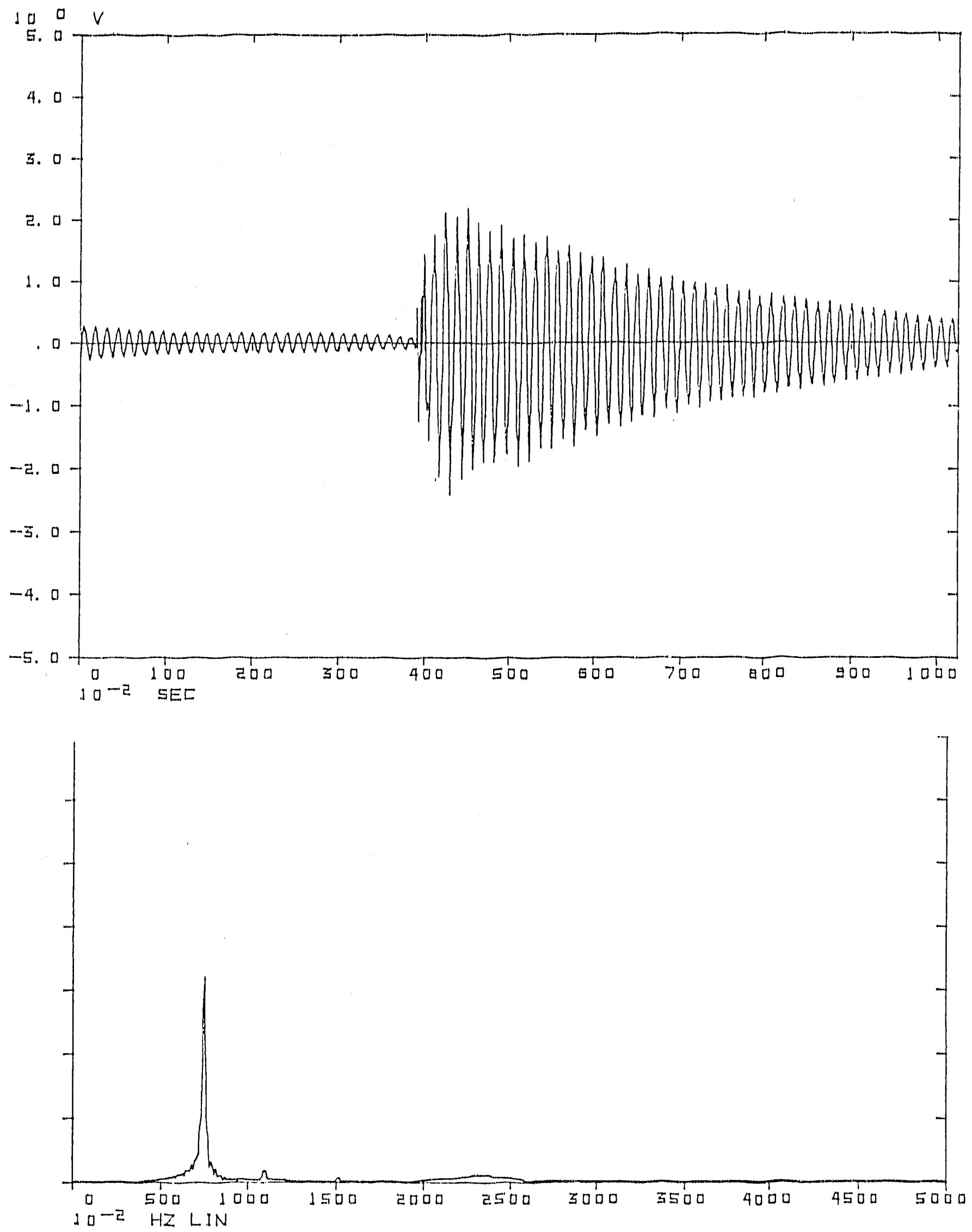

Impulse Excitation on Magnet in $Y$ Direction

Magnet Response in $Y$ Direction

Top-Real Time, Bottem-Frequency 


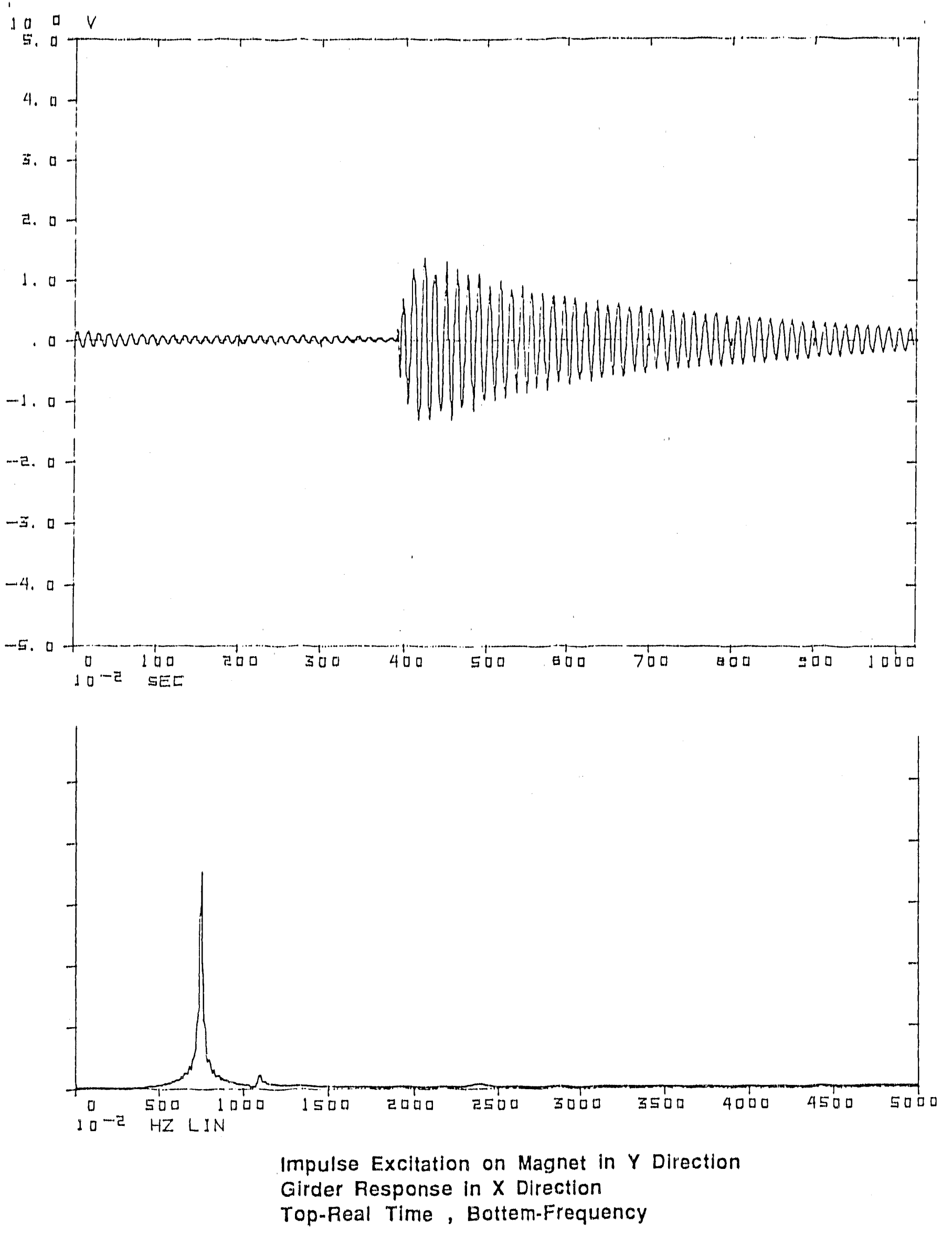




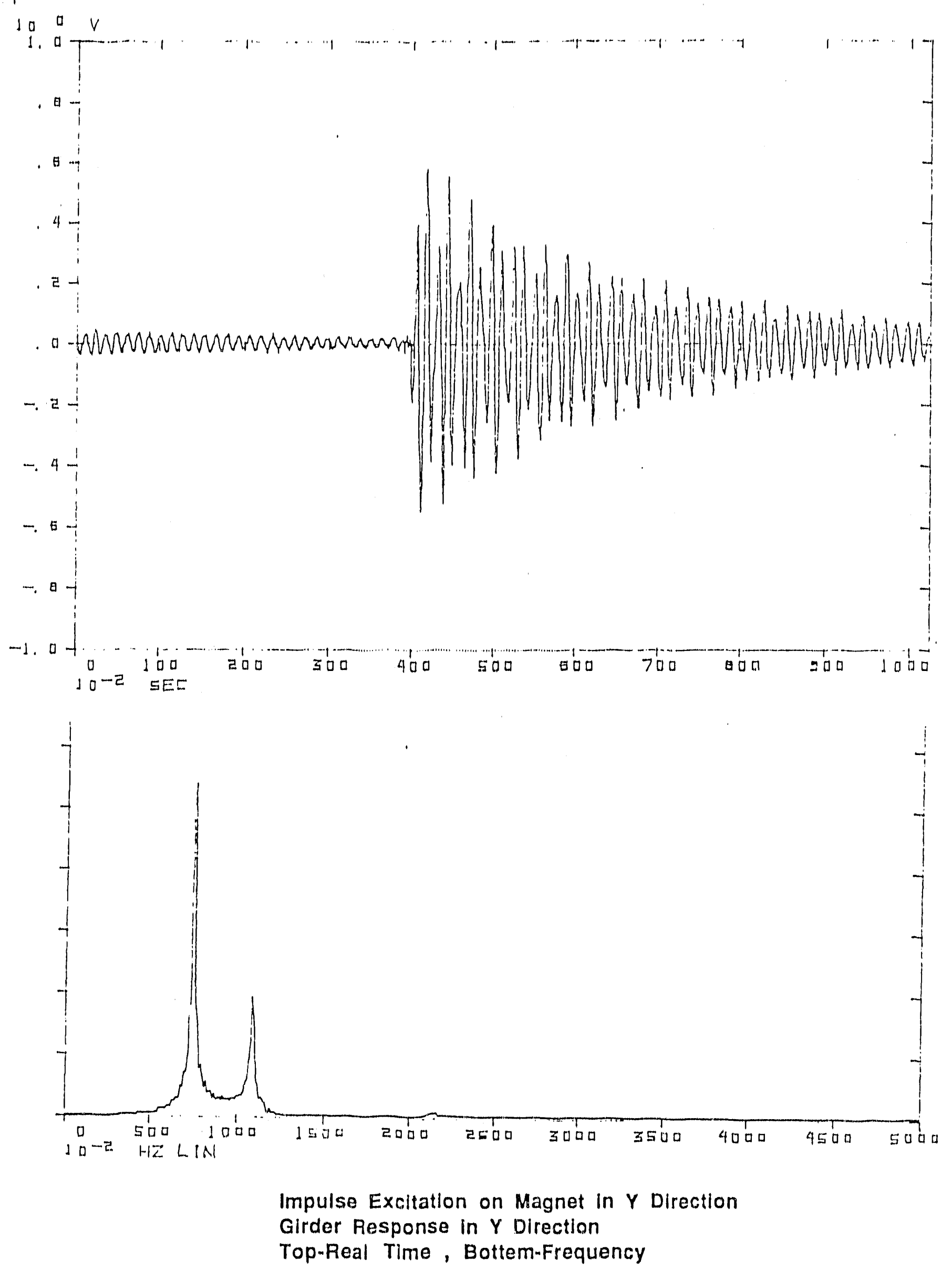




\section{Appendix E}

Ambient Excitation Response for Phase II Tests 
Ambient Disp., Tests 13.15

\begin{tabular}{|c|c|c|c|c|c|c|c|}
\hline & & A & $B$ & $C$ & $D$ & $E$ & $F$ \\
\hline 1 & Test & Location & Wideband & $9.37 \mathrm{~Hz}$. & $111.5 \mathrm{~Hz}$. & $12.9 \mathrm{~Hz}$. & $113.5 \mathrm{~Hz}$. \\
\hline 2 & $13, x$ & ( & 0.1114 & 0.0163 & 0.0175 & 0.0516 & 0.0516 \\
\hline 3 & $13, Y$ & 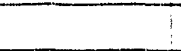 & 0.1318 & 0.0895 & 0.0184 & 0.0597 & 0.0597 \\
\hline 4 & 13.2 & & 0.0637 & 0.01331 & 0.0211 & 0.0139 & 0.0139 \\
\hline 5 & $14, x$ & & 0.1309 & 0.1171 & 0.0081 & 0.0047 & 0.0047 \\
\hline 6 & $14, Y$ & & 0.0455 & 0.0206 & 0.0057 & 0.0035 & 0.0035 \\
\hline 7 & 14.2 & & 0.1764 & 0.0167 & 0.0218 & 0.0049 & 0.0049 \\
\hline 8 & $15, x$ & & 0.2537 & 0.01861 & 0.0323 & 0.2371 & 0.2371 \\
\hline 9 & $15 . Y$ & & 0.1153 & 0.0678 & 0.0128 & 0.0618 & 0.0618 \\
\hline 10 & 15.2 & 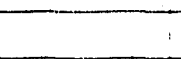 & 0.0708 & 0.0092 & 0.0209 & 0.0326 & 0.0326 \\
\hline 14 & & & & & 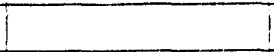 & & \\
\hline 12 & NOTE: & All displa & acements are & in microns, $R M$ & & & 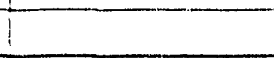 \\
\hline
\end{tabular}

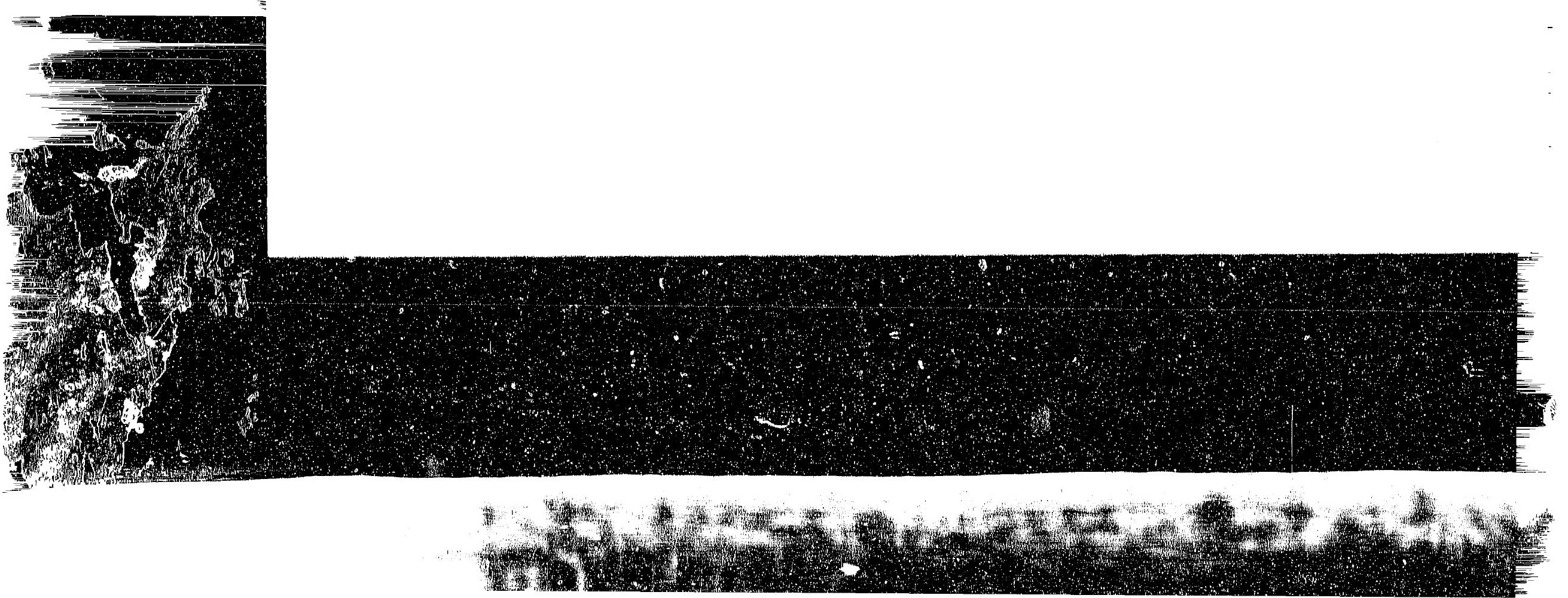


Appendix F

Displacement and Frequency Plots for Phase II Tests 

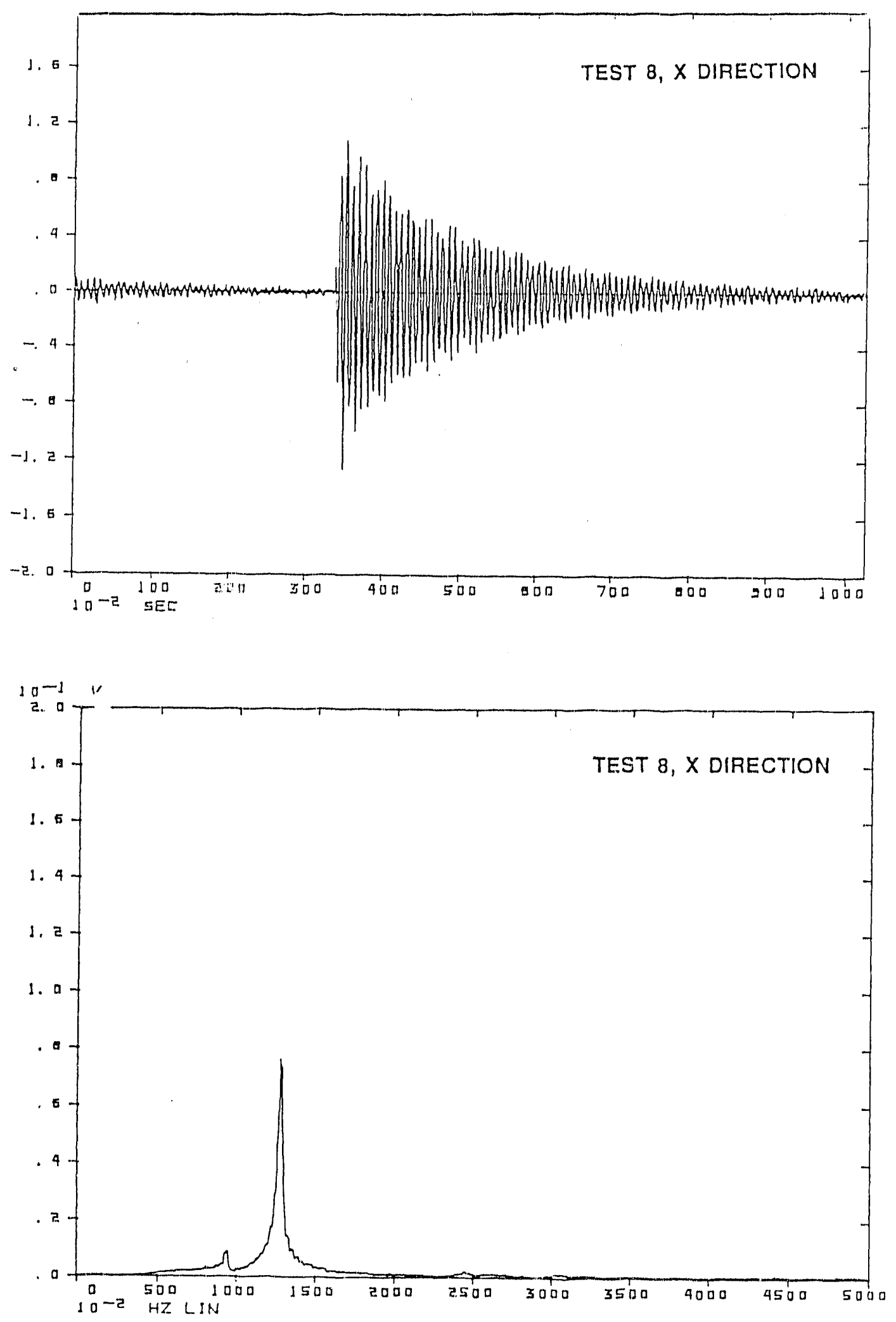

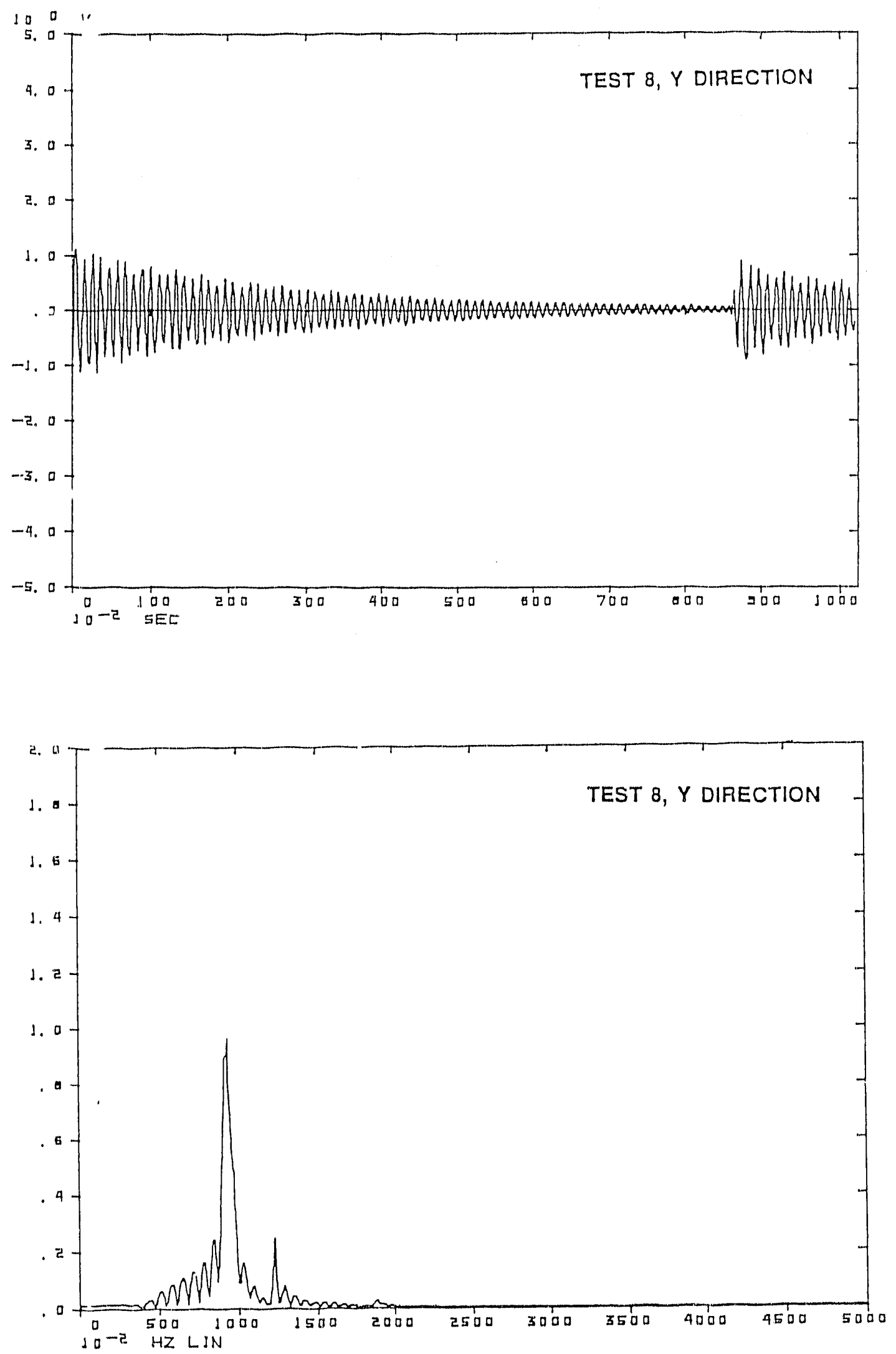

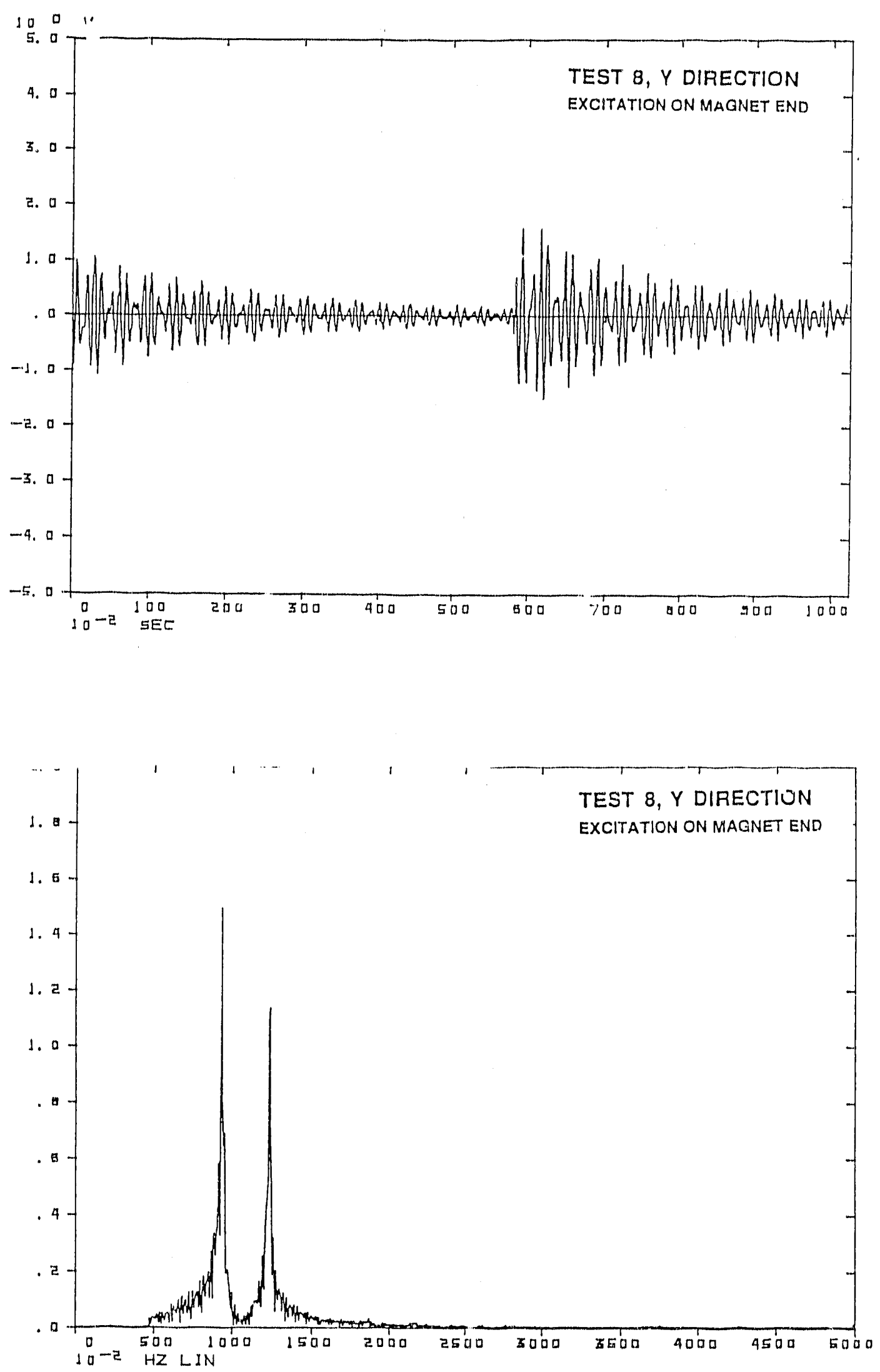

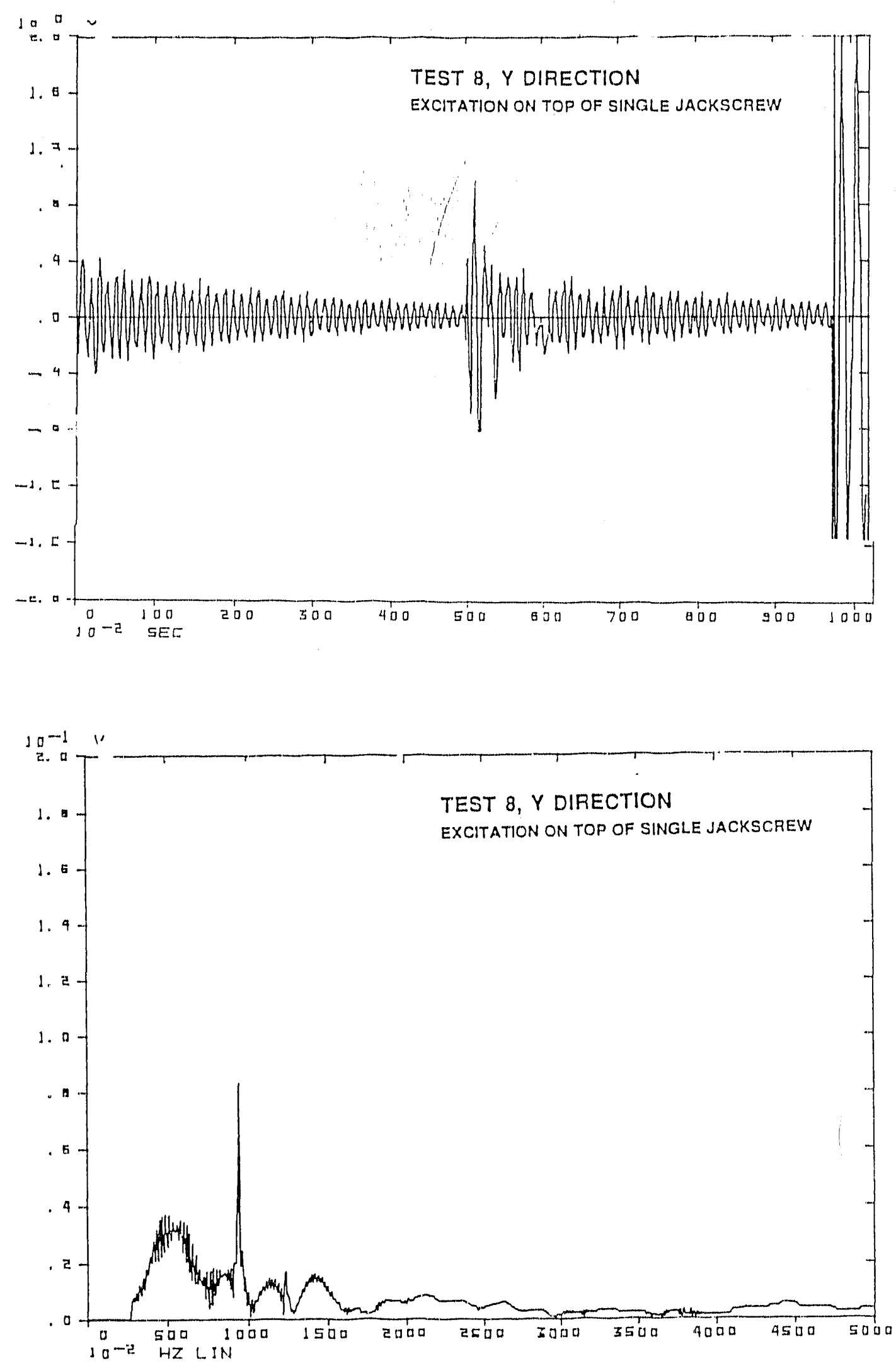

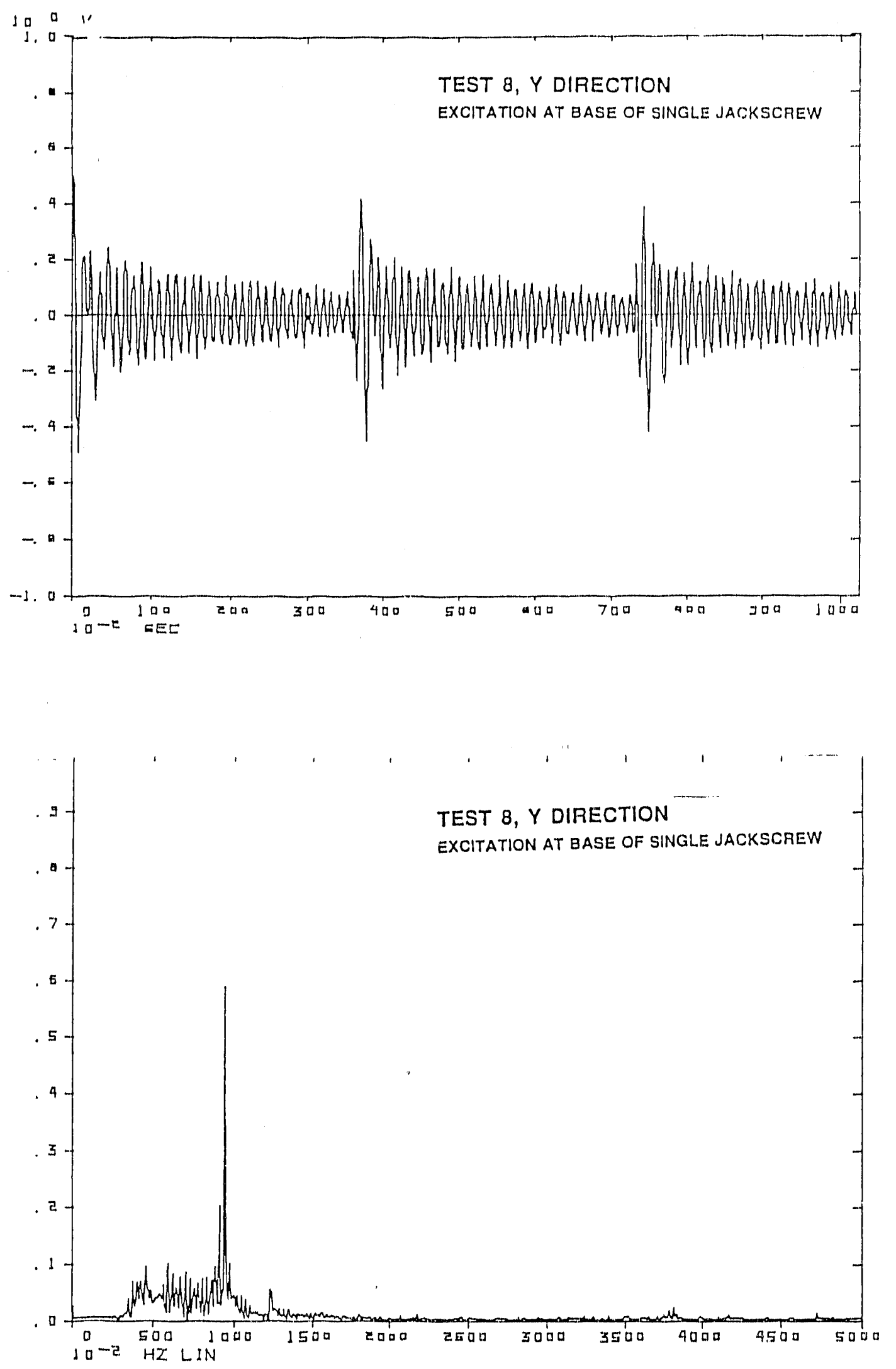

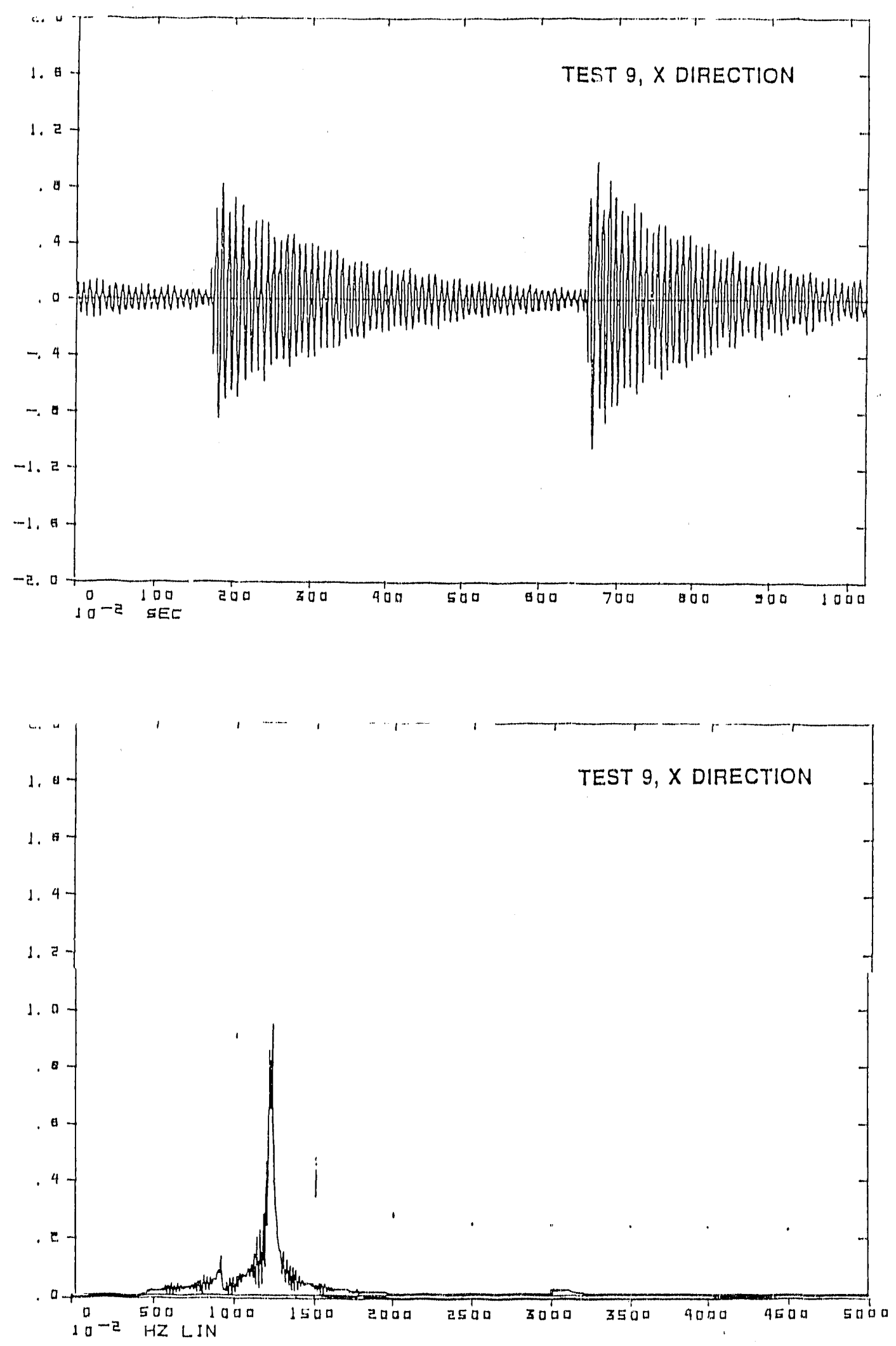

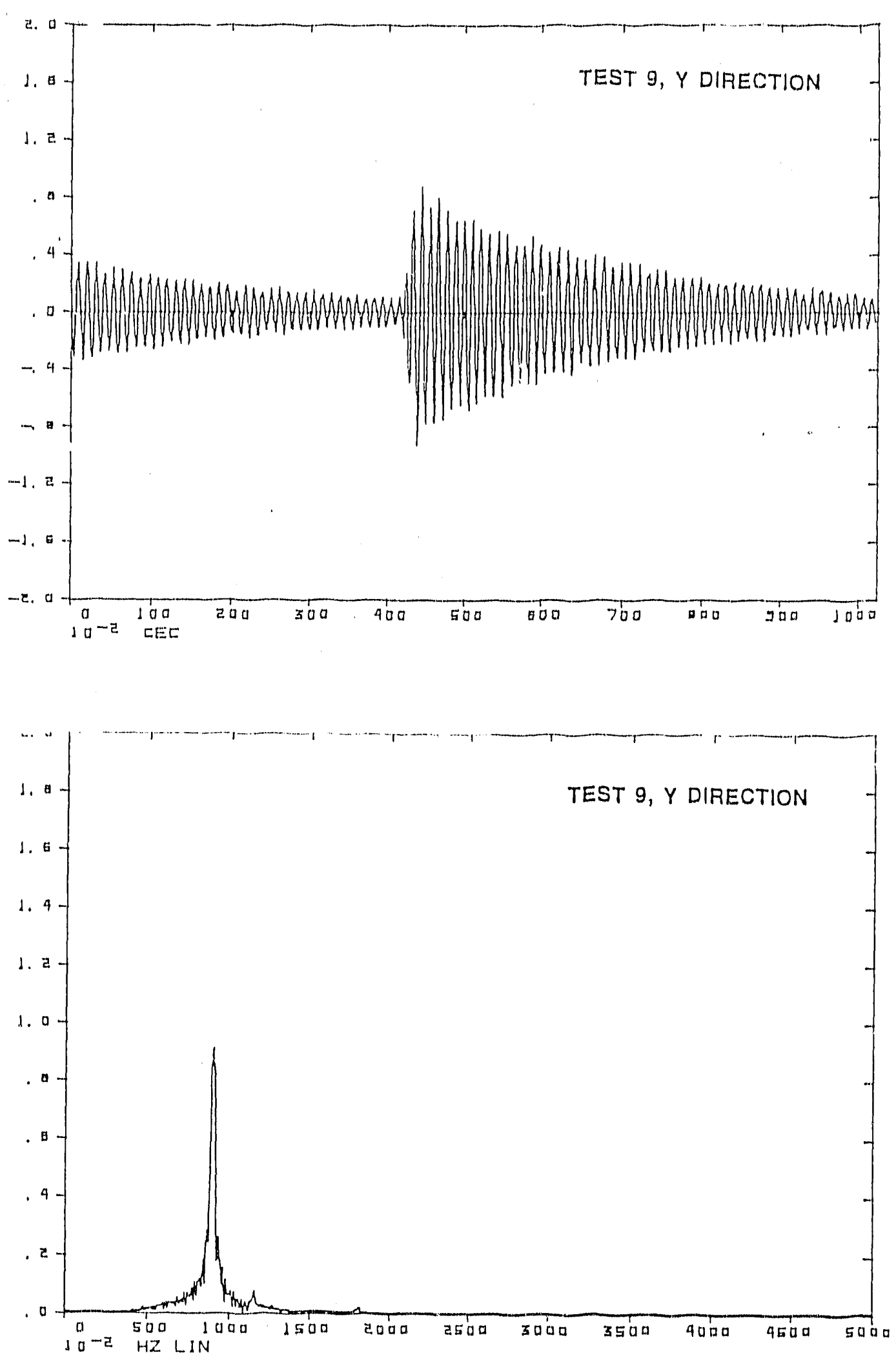

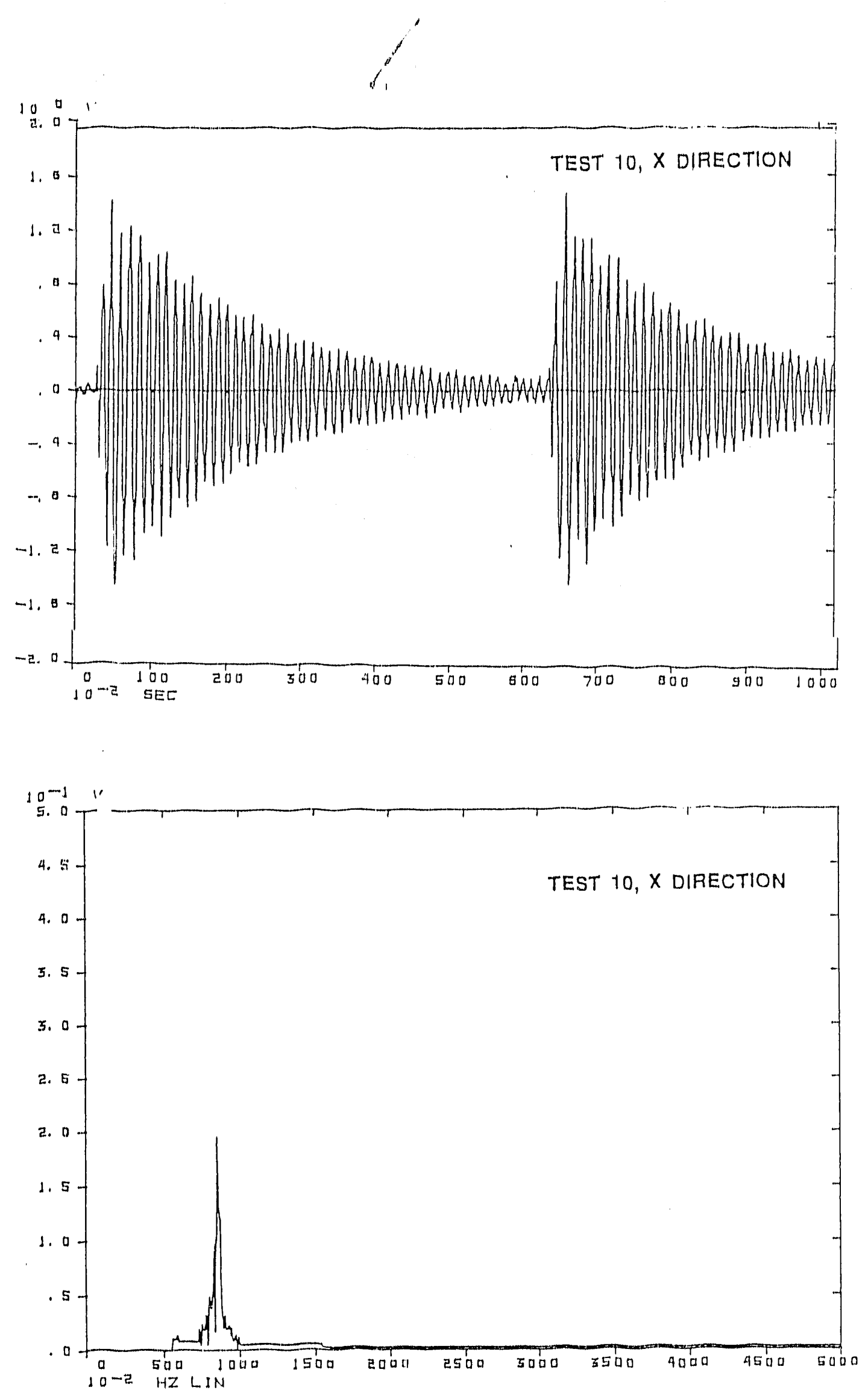

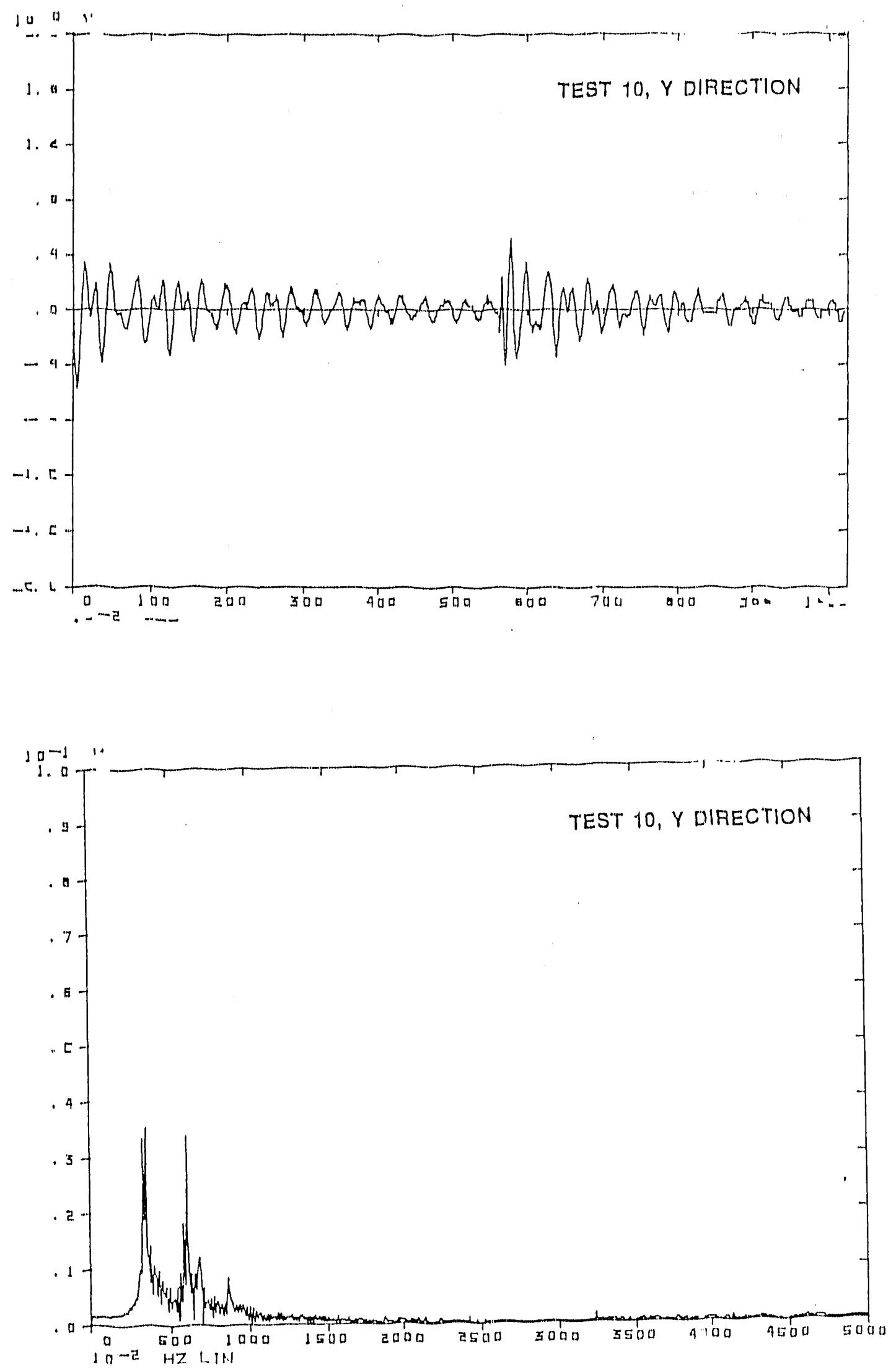

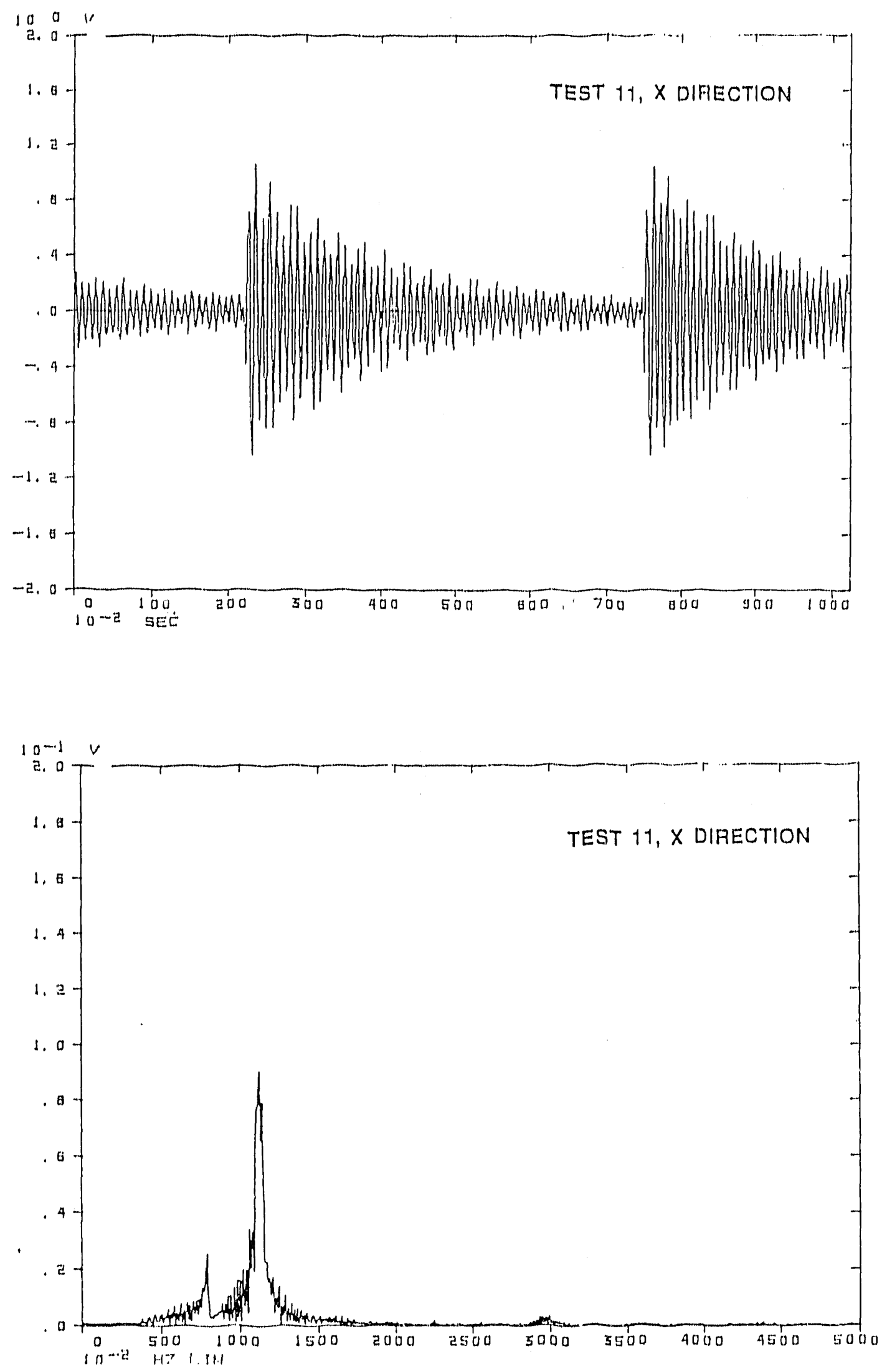

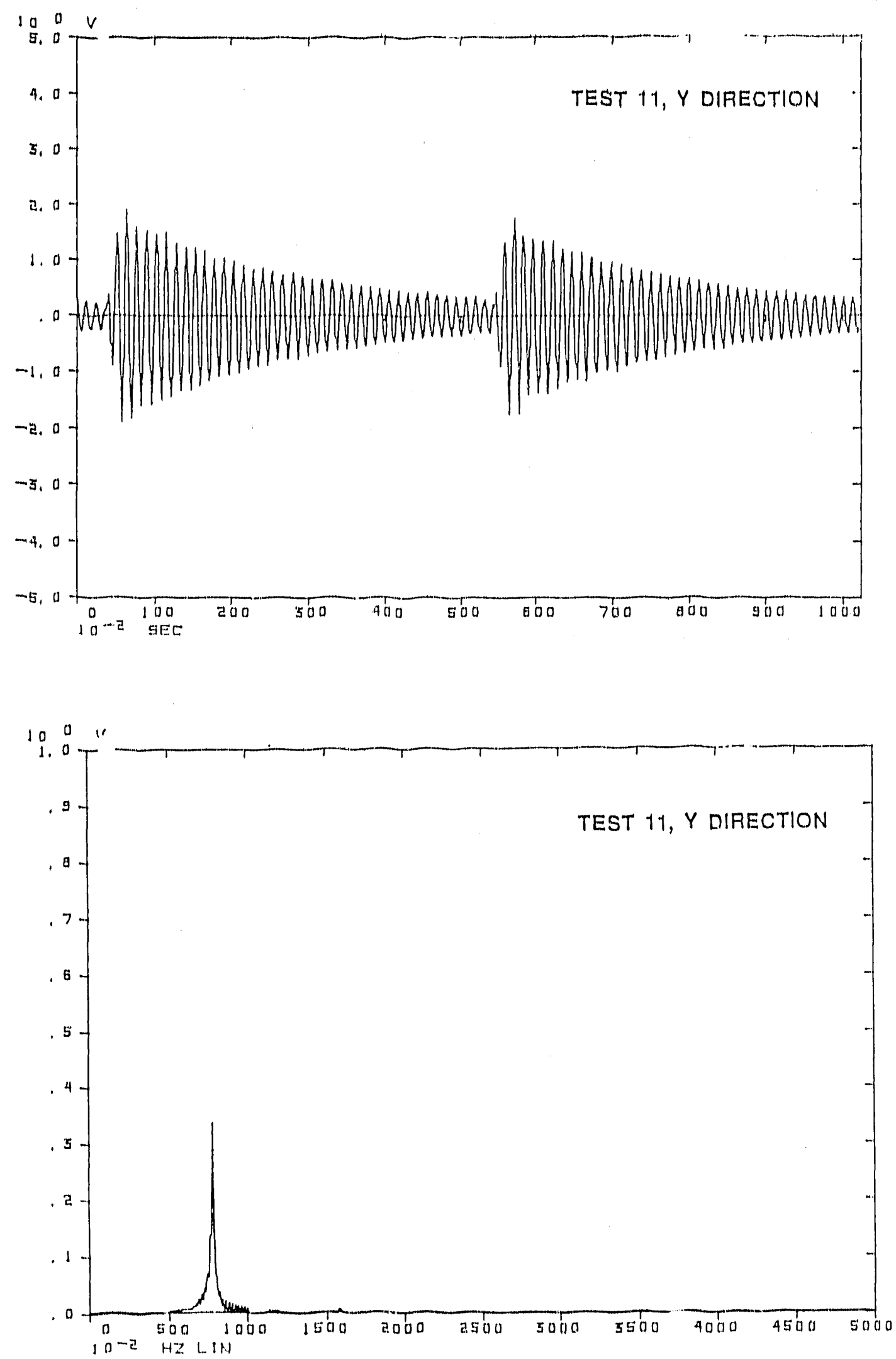

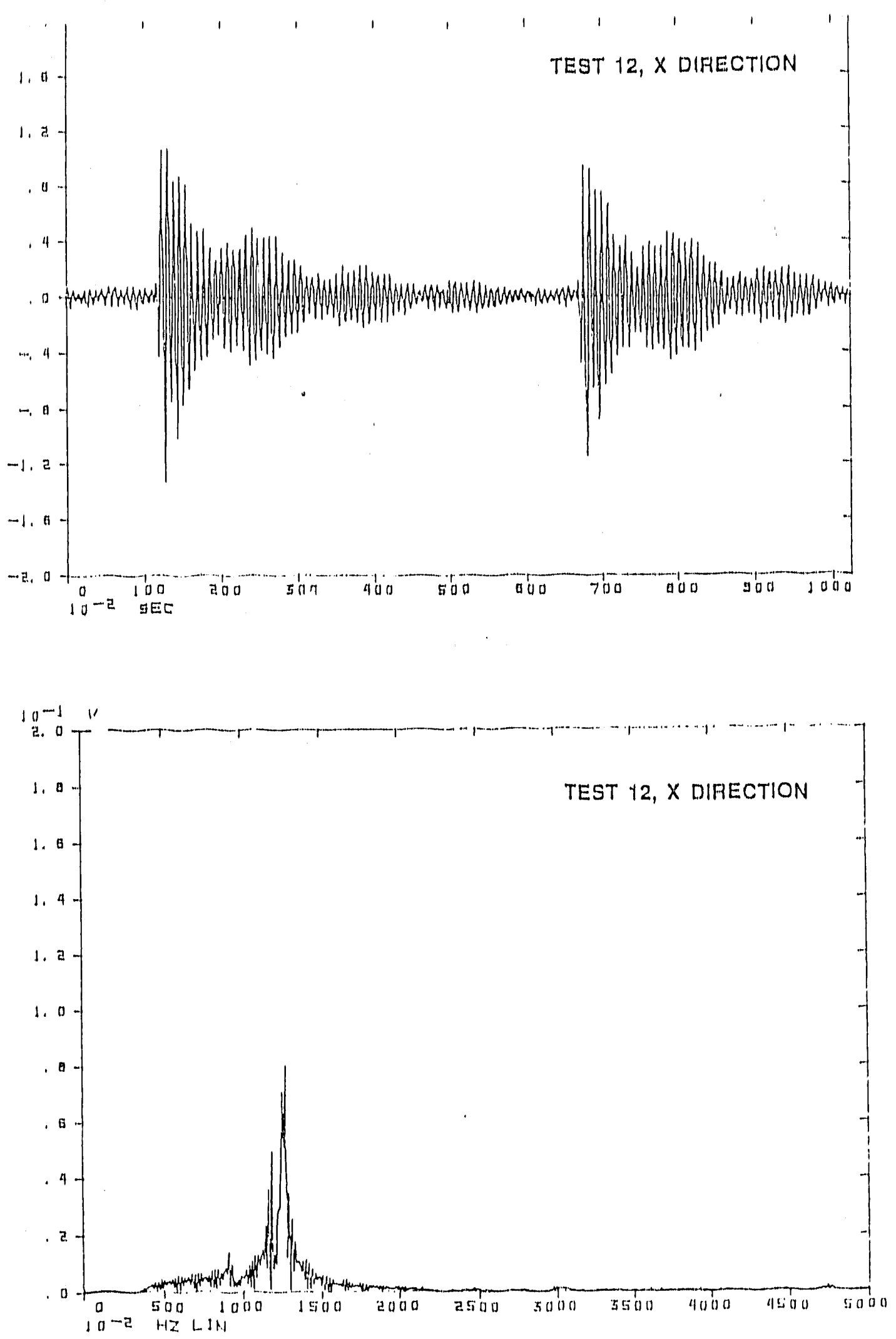

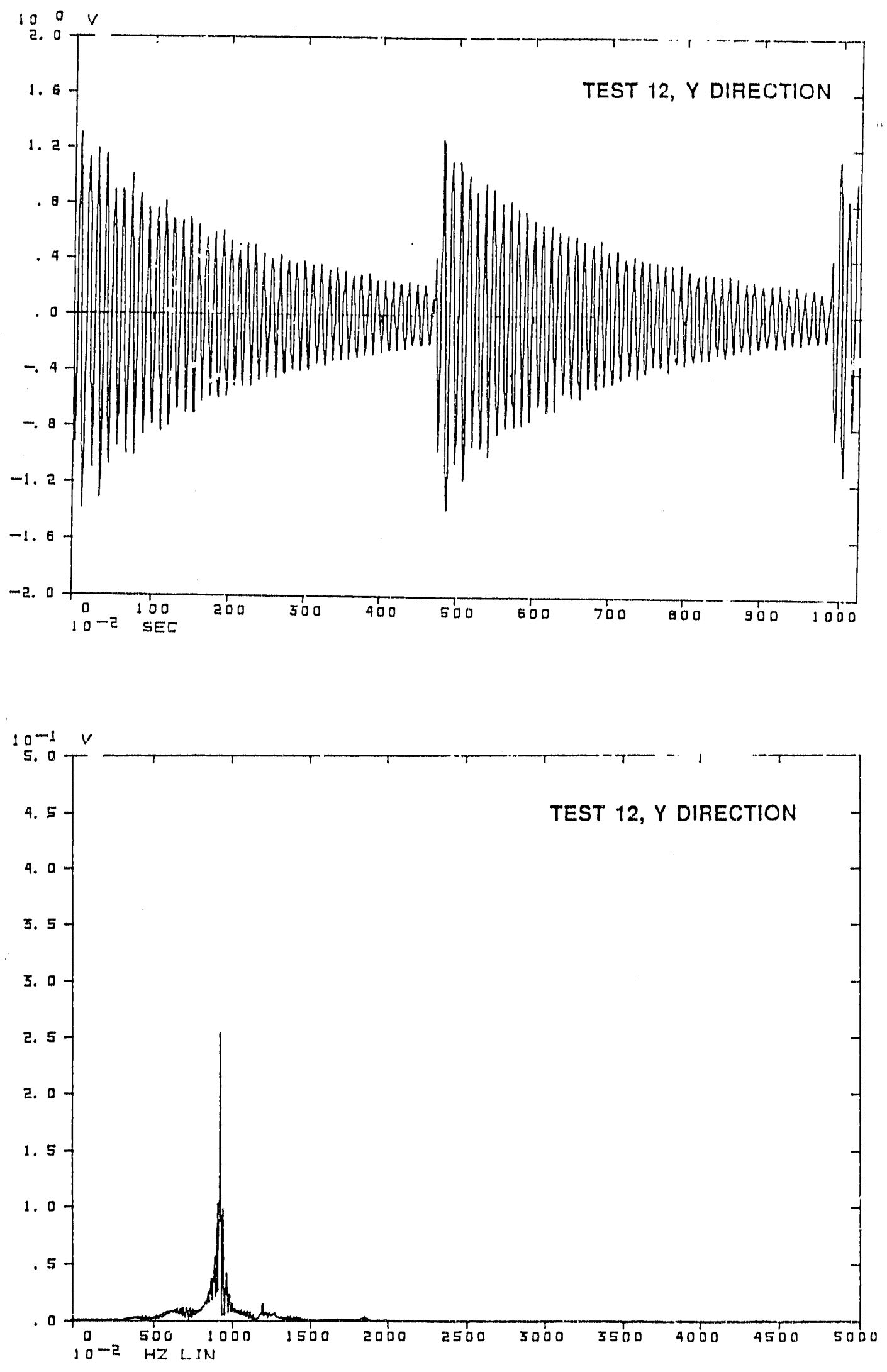

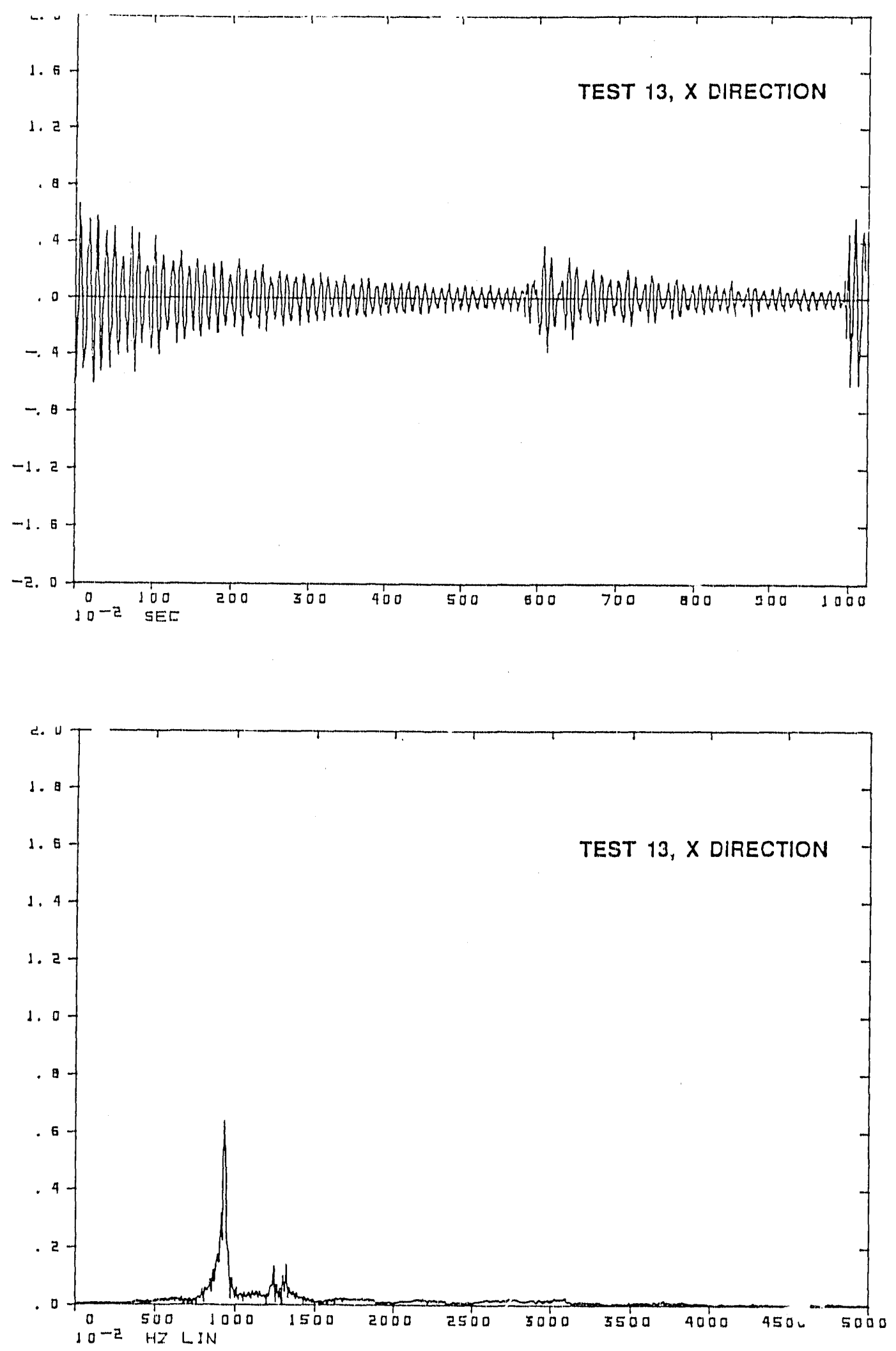

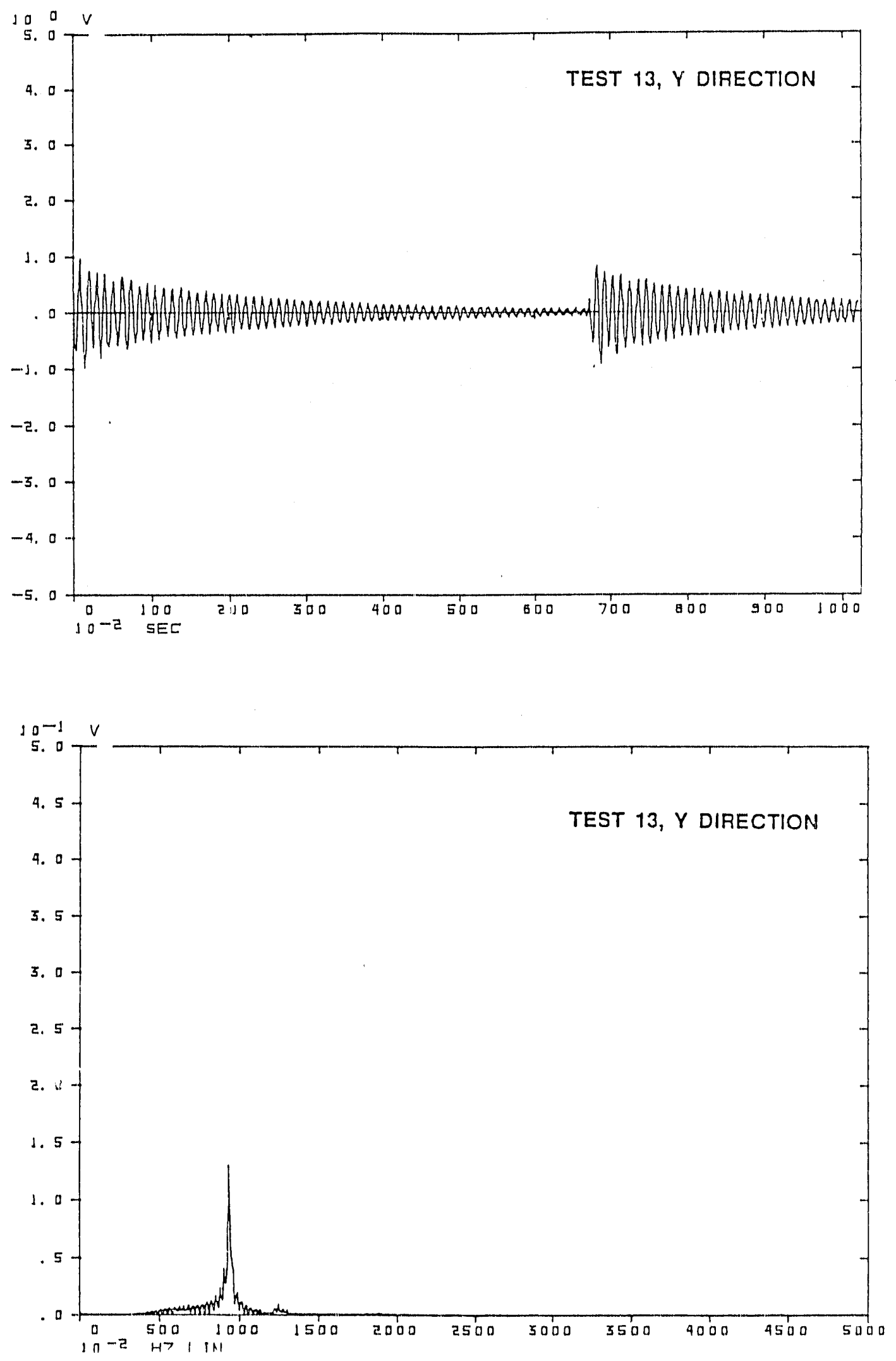

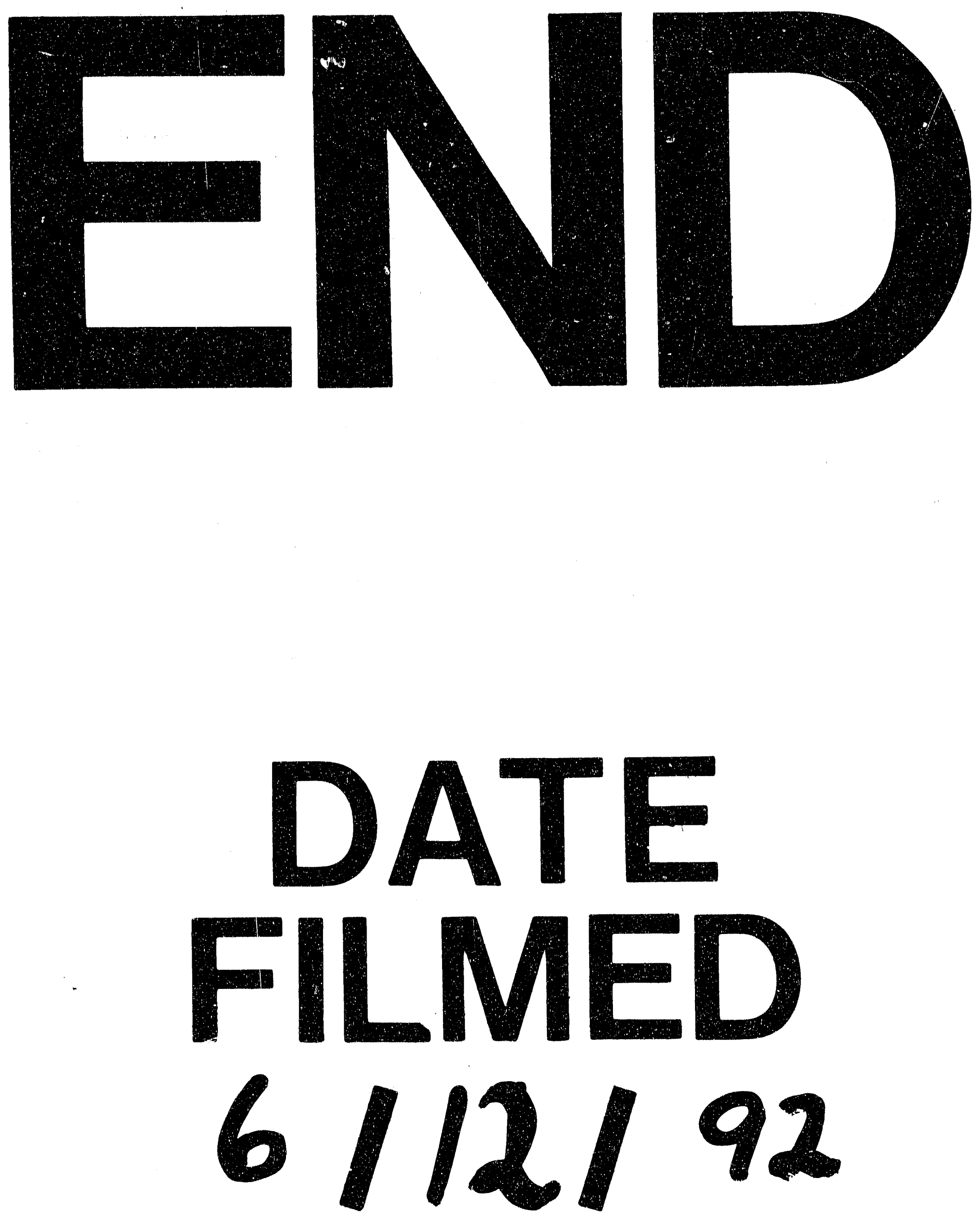

$f$ 
